\title{
Destriping CMB temperature and polarization maps
}

\author{
H. Kurki-Suonio ${ }^{1,2}$, E. Keihänen ${ }^{1}$, R. Keskitalo ${ }^{1,2}$, T. Poutanen ${ }^{2,1,3}$, A.-S. Sirviö ${ }^{1}$, D. Maino ${ }^{4}$, and C. Burigana ${ }^{5,6}$ \\ 1 University of Helsinki, Department of Physics, PO Box 64, 00014 Helsinki, Finland \\ e-mail: hannu.kurki-suonio@helsinki.fi \\ 2 Helsinki Institute of Physics, PO Box 64, 00014 Helsinki, Finland \\ Metsähovi Radio Observatory, Helsinki University of Technology, Metsähovintie 114, 02540 Kylmälä, Finland \\ 4 Dipartimento di Fisica, Universitá di Milano, via Celoria 16, 20131, Milano, Italy \\ 5 INAF/IASF-BO, Istituto di Astrofisica Spaziale e Fisica Cosmica di Bologna, via Gobetti 101, 40129, Bologna, Italy \\ ${ }^{6}$ Dipartimento di Fisica, Università degli Studi di Ferrara, via Saragat 1, 44100 Ferrara, Italy
}

Received 21 April 2009 / Accepted 14 August 2009

\section{ABSTRACT}

\begin{abstract}
We study destriping as a map-making method for temperature-and-polarization data for cosmic microwave background observations. We present a particular implementation of destriping and study the residual error in output maps, using simulated data corresponding to the $70 \mathrm{GHz}$ channel of the PLANCK satellite, but assuming idealized detector and beam properties. The relevant residual map is the difference between the output map and a binned map obtained from the signal + white noise part of the data stream. For destriping it can be divided into six components: unmodeled correlated noise, white noise reference baselines, reference baselines of the pixelization noise from the signal, and baseline errors from correlated noise, white noise, and signal. These six components contribute differently to the different angular scales in the maps. We derive analytical results for the first three components. This study is related to PLANCK LFI activities.
\end{abstract}

Key words. methods: data analysis - cosmology: cosmic microwave background

\section{Introduction}

Construction of sky maps from the time-ordered data (TOD) is an important part of the data analysis of cosmic microwave background (CMB) surveys. For large surveys like PLANCK ${ }^{1}$ (Planck Collaboration 2005), this is a computationally demanding task. Methods which aim at finding the optimal minimum-variance map (Wright 1996; Borrill 1999; Doré et al. 2001; Natoli et al. 2001; Yvon \& Mayet 2005; de Gasperis et al. 2005) are computationally heavy and require large computers. Also, a faster method is needed for Monte Carlo studies to assess systematic effects, noise biases, and error estimates.

Destriping (Burigana et al. 1997b; Delabrouille 1998; Maino et al. 1999, 2002; Revenu et al. 2000; Sbarra et al. 2003; Poutanen et al. 2004; Keihänen et al. 2004; Sutton et al. 2009) is a fast map-making method that removes correlated lowfrequency noise from the TOD utilizing crossing points, i.e., the same locations on the sky observed at different times. Correlated noise is modeled as a sequence of ("uniform") baselines, i.e., constant offsets in the TOD. High-frequency noise (frequency of the same order or higher than the inverse of the baseline length) cannot be modeled this way. Thus the method assumes that the high-frequency part of the noise is uncorrelated (white noise).

In some implementations, a set of base functions (e.g. low order Legendre polynomials) is used instead of just the uniform baseline (Delabrouille 1998; Maino et al. 2002; Keihänen et al. 2004, 2005), or a spline is fitted to the TOD (Ganga 1994).

In this paper we describe one destriping implementation for making temperature and polarization maps of the sky and study the residual errors in the maps. This implementation was originally known as the "Polar" code, and used in the

${ }^{1}$ http://www.rssd.esa.int/index .php?project=PLANCK map-making comparison studies of the PLANCK CTP Working Group (Poutanen et al. 2006; Ashdown et al. 2007a, 2007b, 2009). Polar has now been merged into the "Madam" destriping code. The novel feature in Madam was the introduction of an optional noise prior (noise filter) that utilizes prior information on the noise power spectrum (Keihänen et al. 2005). Polar corresponds to Madam with the noise prior turned off. The results presented in this paper were obtained with the Madam code, with the noise prior turned off. We briefly comment on the effect of the noise prior in Sect. 8. The use and effect of the noise prior will be described in detail in Keihänen et al. (2009).

Destriping errors have been previously analyzed by Stompor \& White (2004) and Efstathiou (2005, 2007).

The TOD can be considered as a sum of signal + white noise + correlated (" $1 / f$ ") noise. If there were no correlated noise, the optimal way to produce a map would be a simple binning of the TOD samples onto map pixels. (We do not address here the question of correcting for the effect of the instrument beam. "Deconvolution" map-making methods that correct for the effect of the beam shape have been developed, Burigana \& Saéz 2003; Armitage \& Wandelt 2004; Harrison et al. 2008, but tend to be computationally very resource intensive. They also alter the noise properties of the maps in a way that is difficult to follow in $\mathrm{CMB}$ angular power spectrum estimation.) Thus the task of a map-making method is to remove the correlated noise as well as possible, with as little effect on the signal and white noise as possible. The difference of the output map from the binned signal + white noise map is thus the residual map to consider to judge the quality of the output map. We divide this residual into six components: unmodeled $1 / f$ noise, $1 / f$ baseline error, white noise reference baselines, white noise baseline error, pixelization noise reference baselines, and pixelization noise 
baseline error. We study the nature of each component, and its dependence on the baseline length.

We have used simulated data corresponding to 1 year of observations with 4 PLANCK LFI $70 \mathrm{GHz}$ detectors (two horns, each with two orthogonally polarized detectors).

For simplicity, we did not include foregrounds in the signal (see Ashdown et al. 2007b, 2009, for effect of foreground signal) or such systematic effects as beam asymmetries, sample integration, cooler noise, or pointing errors (see Ashdown et al. 2009). Even in Ashdown et al. (2009) the simulated data used was still fairly idealized. We are currently working on more realistic simulations.

In Sect. 2 we discuss some early-stage design choices made in the development of our map-making method. Section 3 contains the derivation and description of the method. Section 4 describes the simulated data used to test the method. In Sect. 5 we analyze residual errors in the time domain, and in Sect. 6 in the map domain. In Sect. 7 we discuss the effect of the noise knee frequency, and in Sect. 8 we give a preview of results obtained when a noise prior is added to the method. We mainly consider maps made from a full year of data, but in Sect. 9 we discuss maps made from shorter time segments. In Sect. 10 we summarize our conclusions.

\section{Design choices}

\subsection{Ring set or not}

For a PLANCK-like scanning strategy, where the detectors scan the same circle on the sky many times before the spin axis of the satellite is repointed, an intermediate data structure can be introduced between the TOD and the frequency map. The circles from one repointing period can be coadded to a ring, i.e., averaged to appear just as a single sweep of the circle. In this context it is natural to choose one baseline per ring. Destriping is then performed on this ring set, instead of the original uncoadded TOD. This reduces the memory and computing time requirements by a large factor.

If the scanning is ideal, i.e., the observations (samples) from the different circles of the same ring fall on exactly the same locations on the sky, destriping coadded rings is equivalent to destriping the uncoadded TOD with baseline length equal to the repointing period (i.e., one baseline per ring). In this case it is also almost equal (for map-making purposes) to destriping the uncoadded TOD with baseline length equal to the spin period (i.e. one baseline per circle), see Sect. 6.3.2.

In reality, the spin axis will nutate with some small amplitude, so that the different circles will not scan exactly the same path on the sky. The spin rate is also not exactly constant, and the detector sampling frequency is not synchronized with the spin. Binning the samples first into a ring ("phase binning") (van Leeuwen et al. 2002) and then repixelizing the ring pixels into map pixels after destriping may then introduce some extra smoothing of the data.

We have chosen to sidestep this intermediate structure and to assign the samples directly to map pixels. The baseline length is then not necessarily tied to scan circles and rings, and also data taken during the repointing maneuvers can be used. For short baselines no data compression is possible and map-making is done from the full TOD, requiring large computer memory. For long baselines (many scan circles) the data can be compressed by binning observations directly to map pixels. For baselines one repointing period (one ring) long a similar data compression is achieved as by using the intermediate ring set structure. Whereas the phase-binned ring set is still closely connected to the time domain, our "pixel binning" destroys the time-ordered structure of the ring, and therefore works only with uniform baselines.

This way we have achieved a versatile destriping method, where the baseline length is an adjustable parameter. Shorter baselines can be used in large computers for higher accuracy, whereas longer baselines require less memory and computing time and can be used in medium-sized computers and for Monte Carlo studies. The baseline length is not tied to the scanning strategy, and our destriping method can be applied to any scanning strategy that has crossing points, not just to a PLANCK-like scanning strategy.

However, for a PLANCK-like scanning strategy there is a certain advantage in choosing the baseline length so that an integer number of baselines fits to one repointing period. Baseline segments that extend to two different repointing periods are avoided this way. This is mainly an issue for long baselines (not very much shorter than the repointing period). For baselines shorter than the spin period there seems to be some advantage in choosing the baseline length so that an integer number of baselines fits to one spin period. See Keihänen et al. (2009). In this paper we only consider such choices for baseline length.

\subsection{Crossing points and signal error}

The baselines are estimated from crossing points, i.e., observations falling on the same map pixels at different times. Samples are assigned to pixels based on the pointing of the detector beam center. The beam center may still point at a different location within the same map pixel for different samples. We do not attempt to correct for this effect and this results in a "signal error" in our output maps. The signal error due to in-pixel differences in beam pointing could be largely eliminated in another kind of destriping implementation, where the scanning circles are treated as exact geometrical curves (instead of just a sequence of map pixels), and the observations are interpolated to the exact crossing points of these lines (Revenu et al. 2000). In this case only actual crossings of the scan circles contribute to baseline determination, whereas in our implementation it is enough that two paths pass through the same pixel without actually crossing there. The latter situation is very common, since successive circles are almost parallel.

However, in a realistic situation there are other contributions to signal error that could not be eliminated this way. One such contribution is the different beam orientations of the different observations of the crossing point, as real beams are not circularly symmetric. In Ashdown et al. (2009) elliptic beams were considered for the PLANCK $30 \mathrm{GHz}$ channel and it was found that this had a contribution to the signal error, which was of comparable size or larger.

\section{Destriping technique}

\subsection{Derivation}

The destriping method can be derived from a maximumlikelihood analysis of an idealized model of observations. The signal observed by a detector sensitive to one linear polarization direction is proportional to

$s=I+Q \cos 2 \psi+U \sin 2 \psi$

where $\psi$ is the polarization angle of the detector, and $I, Q$, and $U$ are the Stokes parameters of the radiation coming from the 
observation direction. In the idealized model the time-ordered data (TOD), a vector $\boldsymbol{y}$ consisting of $n_{t}$ samples, is

$y=\mathbf{P} m_{\text {in }}+n$

where $\boldsymbol{m}_{\text {in }}$ (the "input map") represents the sky idealized as a map of $n_{p}$ sky pixels, $\mathbf{P}$ is the pointing matrix, and $\boldsymbol{n}$ is a vector of length $n_{t}$ representing the detector noise. For observations with multiple detectors, the TODs from the individual detectors are appended end-to-end to form the full TOD vector $\boldsymbol{y}$.

Since we are dealing with polarization data, the map $\boldsymbol{m}$ is an object with $3 n_{p}$ elements; for each sky pixel the elements are the $I, Q$, and $U$ Stokes parameters. The pointing matrix $\mathbf{P}$ is of size $\left(n_{t}, 3 n_{p}\right)$. Each row has 3 nonvanishing elements $(1, \cos 2 \psi, \sin 2 \psi)$ at the location corresponding to the sky pixel in which the detector beam center falls for the sample in question (the sample "hits" the pixel). We do not make any attempt at deconvolving the detector beam. Thus the map $\boldsymbol{m}$ represents the sky smoothed with the detector beam and the pixel window function. The pointing matrix spreads the map into a signal TOD Pm.

We divide the TOD into $n_{b}$ segments of equal length $n_{\text {base }}$; $n_{t}=n_{b} n_{\text {base }}$. For each segment we define an offset, called baseline. The baselines model the low-frequency correlated noise component, " $1 / f$ noise", which we want to remove from the data, and we approximate the rest of the noise as white. Thus our idealized noise model is

$\boldsymbol{n}=\mathbf{F} \boldsymbol{a}_{\text {in }}+\boldsymbol{w}$

where the vector $\boldsymbol{a}_{\text {in }}$ (of length $n_{b}$ ) contains the baseline amplitudes and the matrix $\mathbf{F}$, of size $\left(n_{t}, n_{b}\right)$, spreads them into the baseline TOD, which contains a (different) constant value $a_{b}$ ( $\left.b=1, \ldots, n_{b}\right)$ for each baseline segment. Each column of $\mathbf{F}$ contains $n_{\text {base }}$ elements of 1 corresponding to the baseline in question, and the rest of the matrix elements are 0 . The vector $\boldsymbol{w}$ represents white noise, and is assumed to be the result of a Gaussian random process, where the different samples in $w$ are uncorrelated,

$\left\langle w_{t} w_{t^{\prime}}\right\rangle=\sigma_{t}^{2} \delta_{t t^{\prime}}$.

Here \langle\rangle denotes expectation value. The white noise (timedomain) covariance matrix

$\mathbf{C}_{w}=\left\langle\boldsymbol{w} \boldsymbol{w}^{\mathrm{T}}\right\rangle$

is thus diagonal (with elements $\sigma_{t}^{2}$ ), but not necessarily uniform (the white noise variance $\sigma_{t}^{2}$ may vary from sample to sample).

Given the TOD $\boldsymbol{y}$, and assuming we know the white noise variance $\mathbf{C}_{w}$, we want to find the maximum likelihood map $\boldsymbol{m}_{\text {out }}$. We assume no prior knowledge of the baseline amplitudes $\boldsymbol{a}$, i.e., they are assigned uniform prior probability. (The variant of the method, where such prior knowledge is used, is described in Keihänen et al. 2009.)

Given the input map $\boldsymbol{m}$ and the baseline amplitudes $\boldsymbol{a}$, the probability of the data $\boldsymbol{y}$ is

$P(\boldsymbol{y} \mid \boldsymbol{m}, \boldsymbol{a})=\left(\operatorname{det} 2 \pi \mathbf{C}_{w}\right)^{-1 / 2} \exp \left(-\frac{1}{2} \boldsymbol{w}^{\mathrm{T}} \mathbf{C}_{w}^{-1} \boldsymbol{w}\right)$

where $\boldsymbol{w}=\boldsymbol{y}-\mathbf{F} \boldsymbol{a}-\mathbf{P m}$. This is interpreted as the likelihood of $\boldsymbol{a}$ and $\boldsymbol{m}$, given the data $\boldsymbol{y}$ (Press et al. 1992). Maximizing the likelihood is equivalent to minimizing the logarithm of its inverse. We obtain the chi-squared function

$\chi^{2}=-2 \ln P=(\boldsymbol{y}-\mathbf{F} \boldsymbol{a}-\mathbf{P} \boldsymbol{m})^{\mathrm{T}} \mathbf{C}_{w}^{-1}(\boldsymbol{y}-\mathbf{F} \boldsymbol{a}-\mathbf{P m})$

to be minimized. (We dropped the constant prefactor of Eq. (6).) We want to minimize this with respect to both $\boldsymbol{a}$ and $\boldsymbol{m}$.
Minimization of Eq. (7) with respect to $\boldsymbol{m}$ gives the maximum-likelihood map

$\boldsymbol{m}=\left(\mathbf{P}^{\mathrm{T}} \mathbf{C}_{w}^{-1} \mathbf{P}\right)^{-1} \mathbf{P}^{\mathrm{T}} \mathbf{C}_{w}^{-1}(\boldsymbol{y}-\mathbf{F} \boldsymbol{a})$

for a given set of baseline amplitudes $\boldsymbol{a}$.

The symmetric non-negative definite matrix

$\mathbf{M} \equiv \mathbf{P}^{\mathrm{T}} \mathbf{C}_{w}^{-1} \mathbf{P}$

which operates in the map space, is $3 \times 3$ block diagonal, one block $\mathbf{M}_{p}$ for each pixel $p$ :

$\mathbf{M}_{p}=\left(\begin{array}{ccc}\sum_{t} \frac{1}{\sigma_{t}^{2}} & \sum_{t} \frac{\cos 2 \psi_{t}}{\sigma_{t}^{2}} & \sum_{t} \frac{\sin 2 \psi_{t}}{\sigma_{t}^{2}} \\ \sum_{t} \frac{\cos 2 \psi_{t}}{\sigma_{t}^{2}} & \sum_{t} \frac{\cos ^{2} 2 \psi_{t}}{\sigma_{t}^{2}} & \sum_{t} \frac{\cos 2 \psi_{t} \sin 2 \psi_{t}}{\sigma_{t}^{2}} \\ \sum_{t} \frac{\sin 2 \psi_{t}}{\sigma_{t}^{2}} & \sum_{t} \frac{\sin 2 \psi_{t} \cos 2 \psi_{t}}{\sigma_{t}^{2}} & \sum_{t} \frac{\sin ^{2} 2 \psi_{t}}{\sigma_{t}^{2}}\end{array}\right)$,

where the sums run over all samples $t$ that hit pixel $p . \mathbf{M}_{p}^{-1}$ is the white noise covariance matrix for the three Stokes parameters $I$, $Q, U$ in pixel $p . \mathbf{M}_{p}$ can only be inverted if the pixel $p$ is sampled with at least 3 sufficiently different polarization directions $\psi_{t}$, so that all Stokes parameters can be determined. This can be gauged by the condition number of $\mathbf{M}_{p}$. If the inverse condition number rcond (ratio of smallest to largest eigenvalue) is below some predetermined limit, the pixel $p$ is excluded from all maps (as are pixels with no hits), and the samples that hit those pixels are ignored in all TODs. Technically, this is done by setting $\mathbf{M}_{p}^{-1}=0$ for such pixels and $\left(\mathbf{C}_{w}^{-1}\right)_{t t}=0$ for the corresponding samples. $\mathbf{M}$ can then be easily inverted by non-iterative means.

If all $\sigma_{t}$ are equal, $\sigma_{t}^{2} \mathbf{M}_{p}(1,1)$, gives the number of hits (observations) in pixel $p$. Thus $\mathbf{M}$ is sometimes called the $N_{\text {obs }}$ matrix. The optimal distribution of polarization directions $\psi_{t}$ measured from a pixel is one where they are uniformly distributed over $180^{\circ}$ (Couchot et al. 1999). In this case $\mathbf{M}_{p}=$ $\left(n_{\text {hit }, p} / \sigma_{t}^{2}\right) \operatorname{diag}(1,1 / 2,1 / 2)$ and $\mathbf{M}_{p}^{-1}=\left(\sigma_{t}^{2} / n_{\text {hit }, p}\right) \operatorname{diag}(1,2,2)$ giving the maximum possible value rcond $=0.5$.

Substituting Eq. (8) back into Eq. (7) we get this into the form

$\chi^{2}=(\boldsymbol{y}-\mathbf{F} \boldsymbol{a})^{\mathrm{T}} \mathbf{Z}^{\mathrm{T}} \mathbf{C}_{w}^{-1} \mathbf{Z}(\boldsymbol{y}-\mathbf{F} \boldsymbol{a})$,

where we have defined

$\mathbf{Z} \equiv \mathbf{I}-\mathbf{P} \mathbf{M}^{-1} \mathbf{P}^{\mathrm{T}} \mathbf{C}_{w}^{-1}$

Here $\mathbf{I}$ is the unit matrix. The matrix $\mathbf{Z}$ operates in TOD space and is a projection matrix, $\mathbf{Z}^{2}=\mathbf{Z}$. If all $\sigma_{t}$ are equal, $\mathbf{Z}$ is symmetric. In general, $\mathbf{C}_{w}^{-1} \mathbf{Z}$ is symmetric, so that

$\mathbf{C}_{w}^{-1} \mathbf{Z}=\mathbf{Z}^{\mathrm{T}} \mathbf{C}_{w}^{-1}=\mathbf{Z}^{\mathrm{T}} \mathbf{C}_{w}^{-1} \mathbf{Z}$.

We minimize Eq. (11) with respect to $\boldsymbol{a}$ to obtain the maximumlikelihood estimate of the baseline amplitudes $\boldsymbol{a}_{\text {out }}$. It is the solution of the equation

$\left(\mathbf{F}^{\mathrm{T}} \mathbf{C}_{w}^{-1} \mathbf{Z F}\right) \boldsymbol{a}=\mathbf{F}^{\mathrm{T}} \mathbf{C}_{w}^{-1} \mathbf{Z} \boldsymbol{y}$,

where we have used Eq. (13).

The matrix

$\mathbf{D} \equiv \mathbf{F}^{\mathrm{T}} \mathbf{C}_{w}^{-1} \mathbf{Z F}$

on the left-hand side of Eq. (14) operates in the baseline space. It is symmetric but singular. Equation (14) has a solution only if its right-hand side is orthogonal to the null space of $\mathbf{D}$. The solution 
becomes unique when we require it to be orthogonal to the null space too.

The null space of $\mathbf{D}$ contains the vector that gives all baselines the same amplitude. This represents the inability to detect a constant offset of the entire noise stream $\boldsymbol{n}$, because it has the same effect on $\boldsymbol{y}$ as a constant shift in the $I$ of the entire $\boldsymbol{m}_{\text {in }}$ (the monopole). This is of no concern (but should be kept in mind) since the goal is to measure the CMB anisotropy and polarization, not its mean temperature. If the baselines are sufficiently connected by crossing points (two different baseline segments of the TOD hitting the same pixel), there are no other kind of vectors in the null space, so that the dimension of the null space is one. The right-hand side of Eq. (14) is orthogonal to this onedimensional null space, and thus Eq. (14) can now be solved. In practice it is solved by the conjugate gradient method. If the initial guess is orthogonal to the null space, the method converges to a solution that is also orthogonal to the null space. Normally we start with the zero vector as the initial guess to guarantee this. This means that the average of the solved baseline amplitudes is zero. Strictly speaking this holds exactly only when no pixels are excluded from the baseline determination due to their poor rcond.

We write the solution of Eq. (14) as

$\boldsymbol{a}_{\text {out }}=\mathbf{D}^{-1} \mathbf{F}^{\mathrm{T}} \mathbf{C}_{w}^{-1} \mathbf{Z} \boldsymbol{y}$.

$\mathbf{D}^{-1}$ is interpreted as the inverse in this orthogonal subspace. $\mathbf{D}$ and $\mathbf{D}^{-1}$ will act in this subspace only.

Using the maximum likelihood baselines from Eqs. (16) in (8) we get the output map of the destriping method:

$\boldsymbol{m}_{\text {out }}=\mathbf{M}^{-1} \mathbf{P}^{\mathrm{T}} \mathbf{C}_{w}^{-1}\left(\boldsymbol{y}-\mathbf{F} \boldsymbol{a}_{\text {out }}\right)$.

Equations (16) and (17) summarize the destriping method.

Implementation details are discussed in Keihänen et al. (2009).

\subsection{Description}

Let us review the different operations involved:

$\mathbf{P}^{\mathrm{T}} \mathbf{C}_{w}^{-1}$ acts on a TOD $\boldsymbol{y}$ to produce from it a sum map $\mathbf{P}^{\mathrm{T}} \mathbf{C}_{w}^{-1} \boldsymbol{y}$ where each pixel has Stokes parameters representing a sum over observations that hit the pixel,

$I_{p}=\sum_{t \in p} \frac{1}{\sigma_{t}^{2}} y_{t}$

$Q_{p}=\sum_{t \in p} \frac{1}{\sigma_{t}^{2}} \cos 2 \psi_{t} y_{t}$

$U_{p}=\sum_{t \in p} \frac{1}{\sigma_{t}^{2}} \sin 2 \psi_{t} y_{t}$.

Instead of a sum, we should take the average of observations. This is accomplished by $\mathbf{M}^{-1} \mathbf{P}^{\mathrm{T}} \mathbf{C}_{w}^{-1}$, which corresponds to solving the Stokes parameters from the observations hitting this pixel; i.e., without regard for pixel-to-pixel noise correlations. The observations are just weighted by the inverse white noise variance. The resulting map is called the naive map, or the binned map. We shall use the shorthand notation

$\mathbf{B} \equiv \mathbf{M}^{-1} \mathbf{P}^{\mathrm{T}} \mathbf{C}_{w}^{-1}$.

B acts on a TOD to produce from it a binned map. Note that $\mathbf{B P}=\mathbf{I}$.

(It may be better to use the same $\sigma_{t}$ for the two polarization directions of the same horn, to avoid polarization artifacts from systematic effects (Leahy et al. 2009). In case their true noise levels are different, destriping allows also the option of using equal $\sigma_{t}$ in solving baselines, Eq. (16), but the actual $\sigma_{t}$ in the final binning to the map, Eq. (17). We do not study this issue in this paper, as we used simulated data with a constant $\sigma_{t}$.)

We can now see that the effect of

$\mathbf{Z}=\mathbf{I}-\mathbf{P B}$

on a TOD is to bin it to a map, read a TOD out of this map, and subtract it from the original TOD. Thus $\mathbf{Z} \boldsymbol{y}$ represents an estimate of the noise part of $\boldsymbol{y}$. Acting on a TOD constructed from a map as $\mathbf{P m}, \mathbf{Z}$ returns zero, $\mathbf{Z P m}=0$.

Likewise, $\mathbf{F}^{\mathrm{T}} \mathbf{C}_{w}^{-1}$ acts on a TOD to sum up the samples of each baseline segment, weighting each sample by $\sigma_{t}^{-2}$.

The effect of the matrix

$$
\mathbf{D}=\mathbf{F}^{\mathrm{T}} \mathbf{C}_{w}^{-1} \mathbf{Z F}=\mathbf{F}^{\mathrm{T}} \mathbf{C}_{w}^{-1} \mathbf{F}-\mathbf{F}^{\mathrm{T}} \mathbf{C}_{w}^{-1} \mathbf{P B F}
$$

on a baseline amplitude vector $\boldsymbol{a}$ is to produce a TOD $\mathbf{F} \boldsymbol{a}$ containing just these baselines, make a noise estimate $\mathbf{Z F} \boldsymbol{a}$ from this baseline TOD, and calculate then the weighted $\operatorname{sum} \mathbf{F}^{\mathrm{T}} \mathbf{C}_{w}^{-1} \mathbf{Z F}$ of this noise estimate for each baseline. Thus the content of Eq. (14) is to find such a set of baseline amplitudes that these noise estimate sums are the same for the baseline TOD $\mathbf{F} \boldsymbol{a}$ as for the actual input TOD $\boldsymbol{y}$. Thus the solved baselines $\boldsymbol{a}_{\text {out }}$ represent the estimated average noise for each baseline segment of the TOD.

These baselines are then subtracted from the TOD to produce the cleaned TOD $\boldsymbol{y}-\mathbf{F} \boldsymbol{a}_{\text {out }}$, which is then binned to produce the output map

$\boldsymbol{m}_{\text {out }}=\mathbf{B}\left(\boldsymbol{y}-\mathbf{F} \boldsymbol{a}_{\text {out }}\right)$

in Eq. (17).

In a good scanning of the sky the number of hits in each pixel is large. From Eq. (22) we see that $\mathbf{Z}$ contains two parts. The first part I gives each row $t$ a large diagonal element 1 corresponding to the TOD sample this row is acting on. The second part gives this row a large number of small nonzero elements corresponding to all samples $t^{\prime}$ that hit this same pixel. The sum of these elements is -1 so that the row sum is zero. Thus the first and second parts make an equally large contribution, but the second part comes in many small pieces.

The matrix D has a similar structure. The first part (see Eq. (23)) is diagonal containing the sum $\sum_{t \in b} 1 / \sigma_{t}^{2}$ over all samples in the baseline segment $b$. The second part gives to each row $b$ a nonzero element for each baseline $b^{\prime}$ that has a crossing point with $b$. For a good scanning each baseline has a large number of crossing points, so that this second part contributes a large number of small elements to each row.

We define a shorthand notation for the matrix

$\mathbf{A} \equiv \mathbf{D}^{-1} \mathbf{F}^{\mathrm{T}} \mathbf{C}_{w}^{-1} \mathbf{Z}$

which appears in Eq. (16) as $\boldsymbol{a}_{\text {out }}=\mathbf{A} \boldsymbol{y}$. This matrix acts on a TOD and produces from it the set of baseline amplitudes according to the destriping method, Eq. (16). Note that $\mathbf{A F}=\mathbf{I}$, in the sense that $\mathbf{A F} \boldsymbol{a}=\boldsymbol{a}$ for any set of baseline amplitudes, whose average is zero (so that $\boldsymbol{a}$ is orthogonal to the null space). Since $\mathbf{Z}$ is a projection matrix, $\mathbf{A Z}=\mathbf{A}$.

For easy reference, all the matrices introduced are collected in Table 1. The square matrices $\mathbf{C}_{w}, \mathbf{M}, \mathbf{D}$, and $\mathbf{C}_{w}^{-1} \mathbf{Z}$ are symmetric, $\mathbf{C}_{w}$ is diagonal, and $\mathbf{M} 3 \times 3$ block diagonal. $\mathbf{Z}$ is a projection matrix. $\mathbf{D}$ is singular, and $\mathbf{D}^{-1}$ is its inverse in the subspace orthogonal to its null space. The third column in the table refers to the equation in which the matrix was introduced. 
Table 1. Table of matrices.

\begin{tabular}{llll}
\hline \hline Matrix & Size & Eq. & Comment \\
\hline $\mathbf{P}$ & $n_{t} \times 3 n_{p}$ & $(2)$ & pointing matrix \\
$\mathbf{F}$ & $n_{t} \times n_{b}$ & $(3)$ & baselines to TOD \\
$\mathbf{C}_{w} \equiv\left\langle\boldsymbol{w} \boldsymbol{w}^{\mathrm{T}}\right\rangle$ & $n_{t} \times n_{t}$ & $(5)$ & white noise cov. \\
$\mathbf{M} \equiv \mathbf{P}^{\mathrm{T}} \mathbf{C}_{w}^{-1} \mathbf{P}$ & $3 n_{p} \times 3 n_{p}$ & $(9)$ & $N_{\text {obs matrix }}$ To \\
$\mathbf{B} \equiv \mathbf{M}^{-1} \mathbf{P}^{\mathrm{T}} \mathbf{C}_{w}^{-1}$ & $3 n_{p} \times n_{t}$ & $(21)$ & bin TOD to a map \\
$\mathbf{Z} \equiv \mathbf{I}-\mathbf{P B}$ & $n_{t} \times n_{t}$ & $(12)$ & \\
$\mathbf{D} \equiv \mathbf{F}^{\mathrm{T}} \mathbf{C}_{w}^{-1} \mathbf{Z} \mathbf{F}$ & $n_{b} \times n_{b}$ & $(23)$ & \\
$\mathbf{A} \equiv \mathbf{D}^{-1} \mathbf{F}^{\mathrm{T}} \mathbf{C}_{w}^{-1} \mathbf{Z}$ & $n_{b} \times n_{t}$ & $(25)$ & solve baselines \\
$\mathbf{R} \equiv\left(\mathbf{F}^{\mathrm{T}} \mathbf{C}_{w}^{-1} \mathbf{F}\right)^{-1} \mathbf{F}^{\mathrm{T}} \mathbf{C}_{w}^{-1}$ & $n_{b} \times n_{t}$ & $(37)$ & reference baselines \\
\hline
\end{tabular}

Note that all the matrices are constructed from $\mathbf{I}, \mathbf{P}, \mathbf{C}_{w}$, and $\mathbf{F}$. The adjustable parameter in the destriping method is the baseline length $n_{\text {base }}$, which affects the matrix $\mathbf{F}$. $\mathbf{P}$ is determined by the scanning strategy and map pixelization, and $\mathbf{C}_{w}$ by detector noise properties.

\subsection{Destriping error}

One can easily show that

$\mathbf{D}_{b b^{\prime}}=\frac{1}{2} \frac{\partial^{2}\left(\chi^{2}\right)}{\partial a_{b} \partial a_{b^{\prime}} \mid \boldsymbol{a}=\boldsymbol{a}_{\text {out }}, \boldsymbol{m}=\boldsymbol{m}_{\text {out }}}$,

i.e, the matrix $\mathbf{D}$ of Eq. (15) is the Fisher matrix of the baselines. Its inverse gives the covariance of the baseline error $\Delta a \equiv$ $\boldsymbol{a}_{\text {out }}-\boldsymbol{a}_{\text {in }}$. In particular,

$\left(\mathbf{D}^{-1}\right)_{b b}=\left\langle\Delta a_{b}^{2}\right\rangle$.

Ignoring the nondiagonal terms we get an approximation

$\left\langle\Delta a_{b}^{2}\right\rangle \approx\left(\sum_{t \in b} \frac{1}{\sigma_{t}^{2}}\right)^{-1}$.

Assuming the white noise variance stays constant, $\sigma_{t}=\sigma$, over a given baseline, this becomes

$\left\langle\Delta a_{b}^{2}\right\rangle \approx \frac{\sigma^{2}}{n_{\text {base }}}$.

We can understand the approximate result (29) as follows. Destriping solves the baseline amplitude $a_{b}$ from the differences between samples from baseline segment $b$ and from other baseline segments that hit the same pixel. Both the baselines $\boldsymbol{a}_{\text {in }}$ and the white noise contribute to these differences. Without the presence of white noise the baselines could be solved exactly, $\boldsymbol{a}_{\text {out }}=\boldsymbol{a}_{\text {in }}$ (in the idealized model considered in this section), so the error comes from the white noise in the samples. Typically there are many more differences involved than there are samples in the baseline (number of hits per pixel $\gg 2$ ), but samples from the baseline segment $b$ contribute only $n_{\text {base }}$ uncorrelated random variables, with variance $\sigma^{2}$, and their contribution corresponds to the result (29). The total of samples from baseline segment $b$ contribute equally to its amplitude determination than do the total of samples from other baseline segments that hit the same pixels. But the latter contribution comes from a much larger number of uncorrelated random variables, with a similar variance, so it gets averaged out by the larger number and makes a much smaller contribution to the baseline error variance (27). This contribution from the white noise from the crossing samples is ignored in (28) and (29). However, it should add to the baseline error variance, so that Eqs. (28) and (29) are underestimates. See also Efstathiou (2005, 2007).

The approximation (29) corresponds to the white noise reference baseline contribution discussed in Sect. 5. We see in Sect. 6 that, while this approximation is good in the time domain, it is not that good in the map domain.

\section{Simulation}

In this study we tested the destriping method using simulated data that is more realistic than the model used to derive the method in Sect. 3. The TOD was produced using PLANCK LevelS simulation software (Reinecke et al. 2006) as a sum of signal, white noise, and correlated noise (called also $1 / f$ noise),

$\boldsymbol{y}=\boldsymbol{s}+\boldsymbol{n}=\boldsymbol{s}+\boldsymbol{w}+\boldsymbol{n}_{\mathrm{c}}$.

We produced the three time streams, $\boldsymbol{s}, \boldsymbol{w}$, and $\boldsymbol{n}_{\mathrm{c}}$ separately, so we could analyze the effect of each component on the destriping error. The TOD represented 1 full year $(366 \mathrm{~d})$ of data from 4 polarized PLANCK $70 \mathrm{GHz}$ detectors (LFI-19a, LFI-19b, LFI-22a, LFI-22b).

\subsection{Signal}

We considered the $\mathrm{CMB}$ signal only; no foregrounds were included in the simulation. Detector pointings $\theta_{t}, \phi_{t}, \psi_{t}$ for each sample $t$ were produced to imitate a realistic scanning strategy. We used a set of input spherical harmonic coefficients $a_{\ell m}^{T}, a_{\ell m}^{E}$ to represent the sky. The signal sample $s_{t}$ was then produced by the convolution of a circularly symmetric Gaussian beam (fwhm $=$ $12.68^{\prime}$ ) centered at this pointing with the input $a_{\ell m}^{T, E}$ (Wandelt \& Górski 2001; Challinor 2000). Thus the $I, Q$, and $U$ (Eq. (1)) of the signal parts of different samples hitting the same pixel are different, as $\theta_{t}, \phi_{t}$ can vary within the pixel.

To produce the input $a_{\ell m}^{T, E}$ we used the $\mathrm{CAMB}^{2}$ code to produce the theoretical angular power spectra $C_{\ell}^{T T}, C_{\ell}^{T E}, C_{\ell}^{E E}$ for the Friedmann-Robertson-Walker universe with cosmological parameter values $\Omega_{0}=1, \Omega_{\Lambda}=0.7, \omega_{\mathrm{m}}=0.147, \omega_{b}=0.022$, $\tau=0.1$, and with scale-invariant $(n=1)$ adiabatic primordial scalar perturbations with amplitude $5 \times 10^{-5}$ for the curvature perturbations. A realization $\left\{a_{\ell m}^{\mathrm{T}}, a_{\ell m}^{E}\right\}$ was then produced from these spectra. Effects of gravitational lensing were ignored, and therefore there is no $B$ mode polarization in the input spectrum.

Figure 1 shows the input $C_{\ell}$ as well as the $C_{\ell}$ of the binned (noiseless) signal map $\mathbf{B} s$ made from the simulated TOD.

Figure 2 shows the binned signal maps $\mathbf{B} \boldsymbol{s}$, and Figs. 3 and 4 zoom into two $10^{\circ} \times 10^{\circ}$ regions to reveal small-scale detail. Since the signal contains only $E$ mode polarization, $Q$ shows structures elongated along lines of latitude and longitude, whereas $U$ shows structures elongated $45^{\circ}$ away from them (see Fig. 4).

In this paper we keep using these same two $10^{\circ} \times 10^{\circ}$ regions, one near the ecliptic north pole, one near the ecliptic, to show map detail. We show all maps in the ecliptic coordinate system. This coordinate system is good for showing map-making related systematics, since the scanning direction is mostly close to the ecliptic meridians.

\subsection{Scanning}

The sky scanning strategy was cycloidal (Dupac \& Tauber 2005): the satellite spin axis was repointed at $1 \mathrm{~h}$ intervals,

2 http://camb.info 

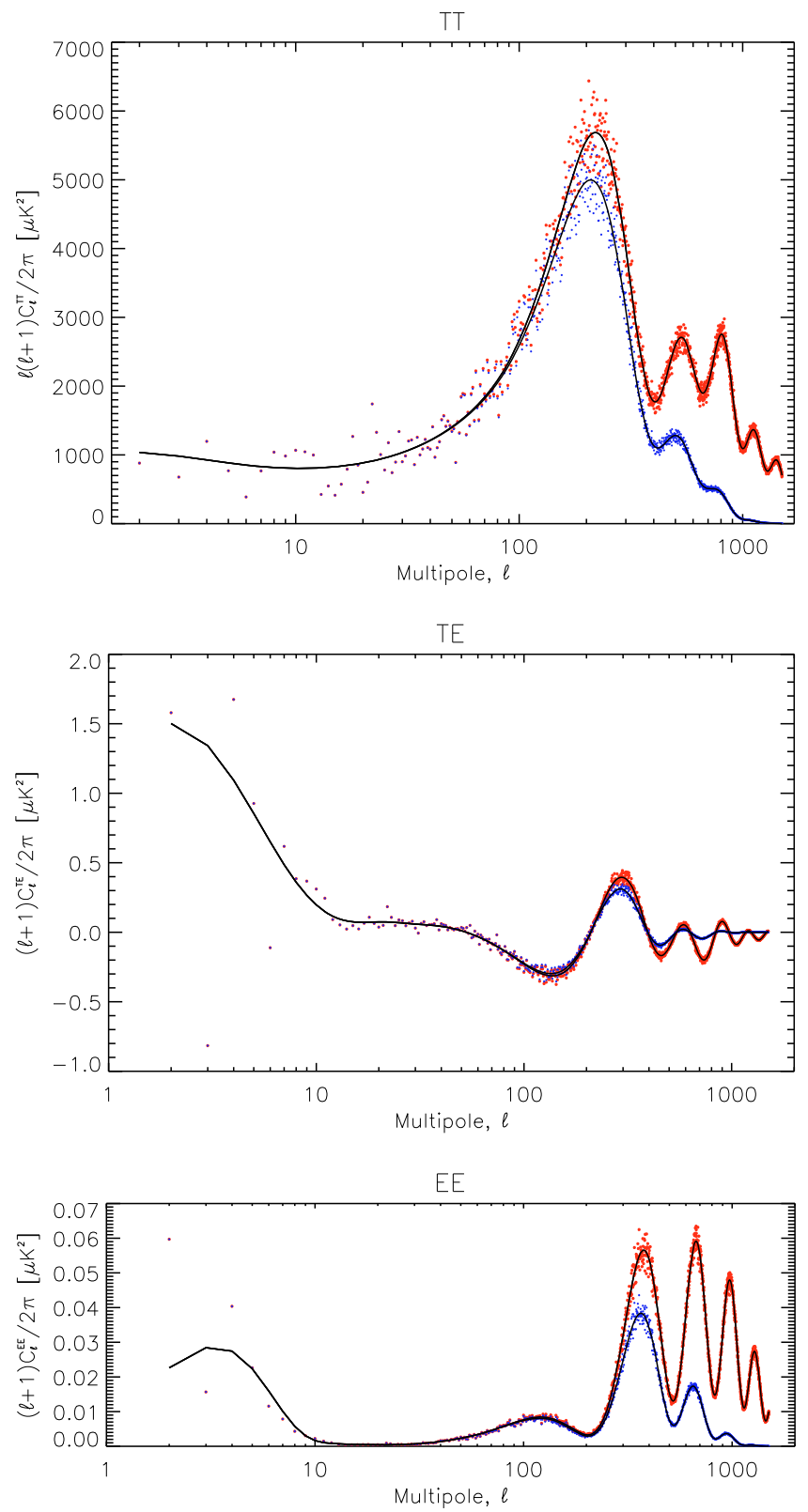

Fig. 1. Input angular power spectrum. Black lines show the theoretical $C_{\ell}$ produced by CAMB, and the beam-and-pixel-smoothed version of it. Red dots show the $C_{\ell}$ of the $a_{\ell m}$ realization. Blue dots show the $C_{\ell}$ calculated from the binned noiseless map.

causing it to make a clockwise circle (radius $7.5^{\circ}$ ) around the anti-Sun direction in 6 months. When the spin axis is north of the ecliptic, the motion along the circle subtracts from the motion of the anti-Sun direction on the sky, making the repointing step shorter; and when the spin axis is south of the ecliptic, the two motions add up, making the repointing step longer.

Random errors $\left(\mathrm{rms}=1.3^{\prime}\right)$ were added to the repointing. Between repointings the spin axis nutated at an amplitude related to the repointing error. The mean nutation amplitude was $1.6^{\prime}$. The nutation was dominated by a combination of two periods, $\sim 45 \mathrm{~s}$ and $\sim 90 \mathrm{~s}$. In reality, the repointing errors and nutation amplitudes are expected to be smaller. Thus effects of nutation and small-scale variations in the map pixel hit count appear somewhat exaggerated in this study.

The satellite rotated clockwise (i.e., spin vector pointing away from the Sun $)$ at about $1 \mathrm{rpm}\left(f_{\text {spin }} \approx 1 / 60 \mathrm{~Hz}\right)$; spin

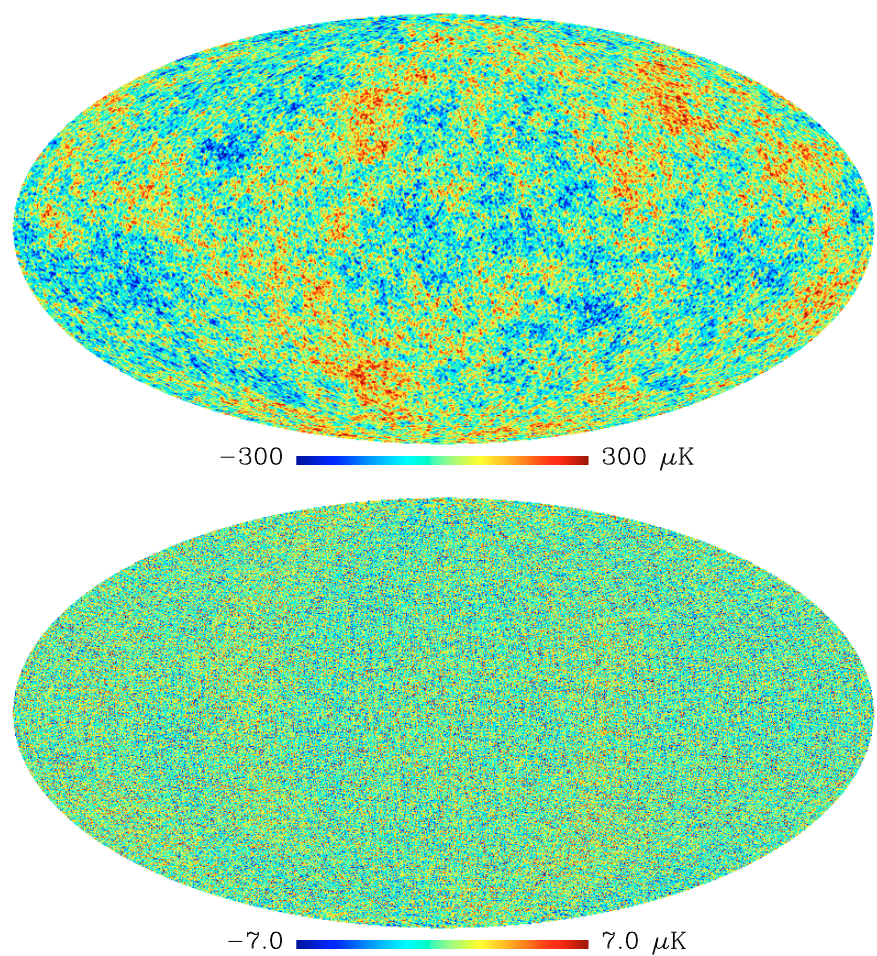

Fig. 2. Binned CMB signal map, $I$ and $Q$, full sky. All maps in this paper are shown using the ecliptic coordinate system.
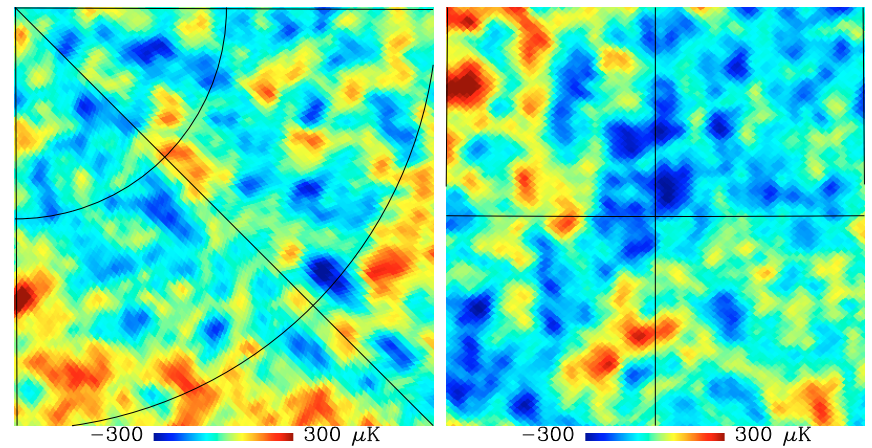

Fig. 3. Binned CMB temperature $(I)$ signal maps from two $10^{\circ} \times 10^{\circ}$ regions. Left column: near the ecliptic North Pole (centered at $\theta=7^{\circ}$, $\phi=-90^{\circ}$ ). Right column: near the ecliptic (centered at $\theta=85^{\circ}, \phi=5^{\circ}$ ).

rate variations ( $\left.\mathrm{rms} 0.1^{\circ} / \mathrm{s}\right)$ around this nominal rate were chosen randomly at each repointing. (In reality, the spin rate variations are expected to be smaller.) Coupled with the $60 \mathrm{~s}$ spin period, the $45 \mathrm{~s}$ and $90 \mathrm{~s}$ nutation periods produce a 3 -min periodicity in the detector scanning pattern.

We simulated 4 detectors corresponding to 2 horns (19 and 22). The detectors were pointed $\theta_{\text {det }}=87.77^{\circ}$ away from the spin axis, causing them to draw almost great circles on the sky, the " 22 " trailing the "19" detectors by $3.1^{\circ}$, but following the same path. The a and b detectors of each horn shared the same pointing but had different polarization directions by exactly $90^{\circ}$. The polarization directions of the 19 and 22 detector pairs differed from each other by $44.8^{\circ}$.

The cycloidal scanning causes the detector scanning rings to form caustics around the ecliptic poles, where nearby scanning rings cross (see Fig. 5). A large number of ring crossings cluster at the four corners of these caustics. For destriping, such a clustering, where very many crossing points fall on the same pixel, is disadvantageous, since there is less independent information 

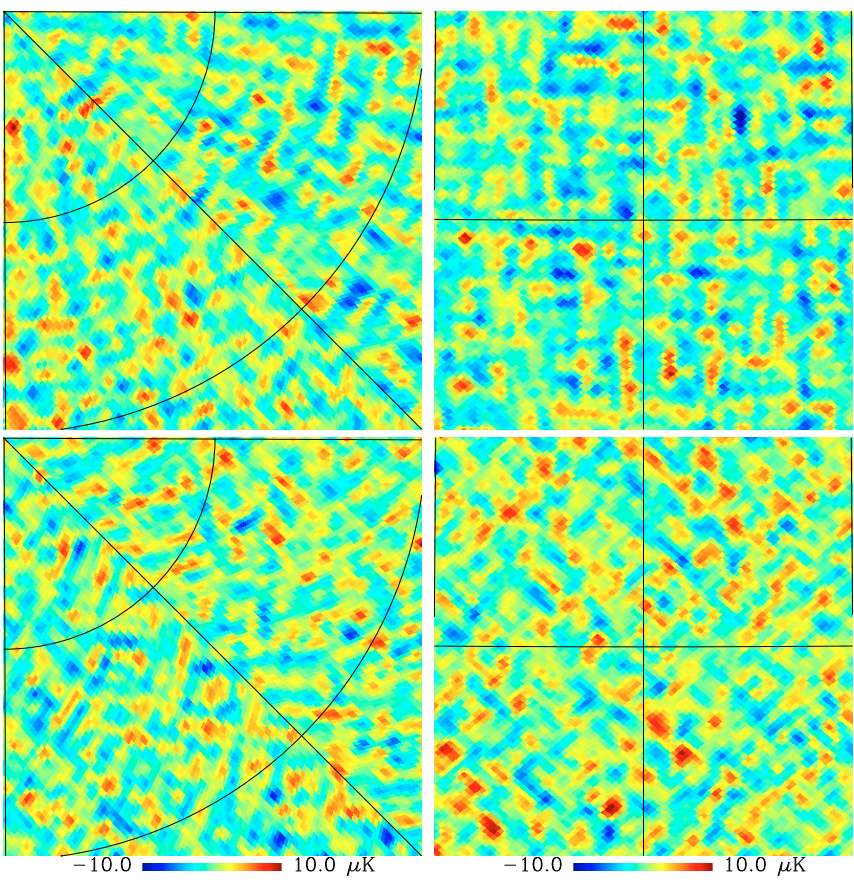

Fig. 4. Same as Fig. 3, but for the Stokes parameters $Q$ (top) and $U$ (bottom).
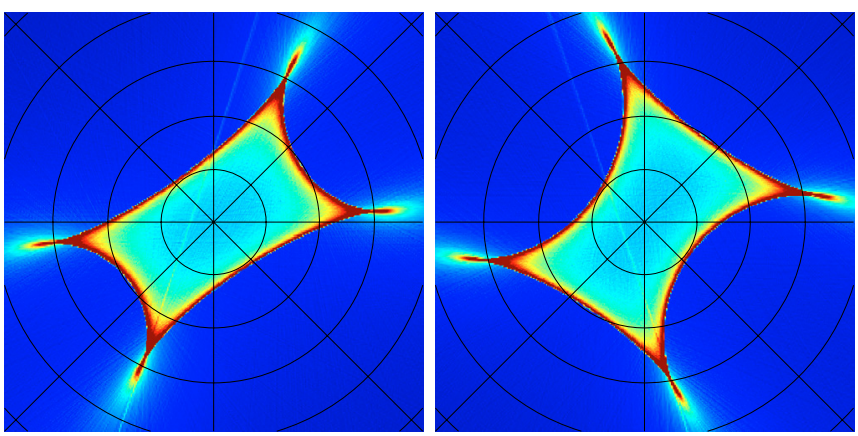

Fig. 5. Hit map for the 1-year simulation. We show regions around the ecliptic North Pole (left) and South Pole (right). The color scale is linear and goes from zero (blue) to 50000 (red). Lines of latitude are drawn at $5^{\circ}$ intervals.

available for solving the baselines of these rings. The clustering occurs when the curvature of the path of satellite pointing on the sky equals the curvature of the scanning circle. The curvature changes sign when the spin axis is close to the ecliptic, but slightly north of it, and the clusterings near the north and south ecliptic poles occur a little bit before and after that. Conversely, the crossing points are spread more widely along the caustics when the spin axis is near its north or south extrema.

The sampling frequency was $f_{\text {sample }}=76.8 \mathrm{~Hz}$, and each sample was simulated as an instantaneous measurement (no integration along scan direction). The baseline length $n_{\text {base, }}$ is given in time units as $t_{\text {base }}=n_{\text {base }} / f_{\text {sample }}$ in the following. The beam center moves on the sky at an angular velocity $\omega_{\text {scan }}=$ $360^{\circ} f_{\text {spin }} \sin \theta_{\text {det }} \approx 6^{\circ} / \mathrm{s}$, so that one baseline corresponds to a path of length $\theta_{\text {base }}=\omega_{\text {scan }} t_{\text {base }}$ on the sky. The samples are separated by $\theta_{\mathrm{s}}=\omega_{\text {scan }} / f_{\text {sample }} \approx 4.68^{\prime}$ on the sky. The length of the simulated TOD was $n_{t}=4 \times 366 \times 24 \times 3600 \mathrm{~s} \times$ $f_{\text {sample }}=9714401280$.

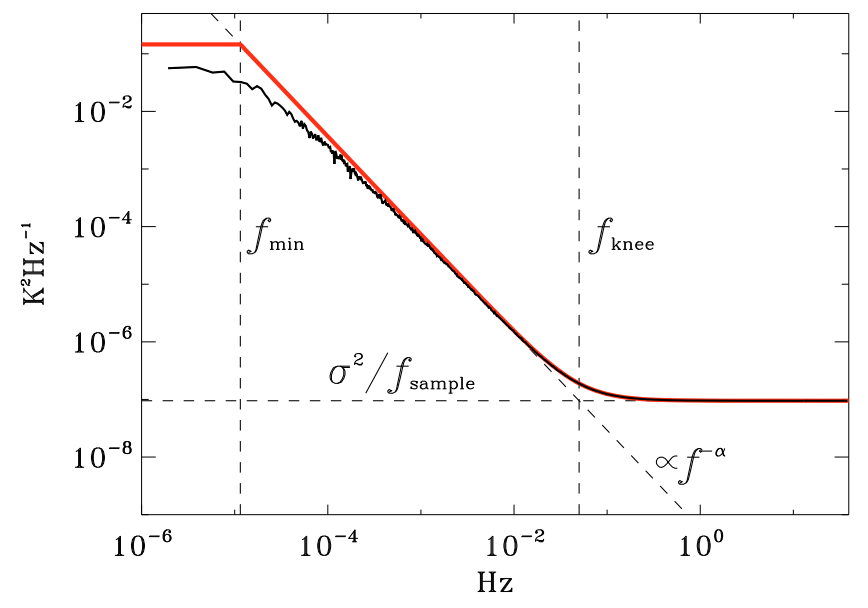

Fig. 6. Power spectrum of the noise stream $\boldsymbol{n}$ (black). The spectrum was calculated from the detector 19a noise stream by dividing it into 6-day pieces, obtaining their spectra separately, taking their average, and binning the spectrum into 800 logarithmic bins, to get a smooth curve. The red curve is the noise model.

\subsection{Noise}

The noise part was produced as a sum of white and correlated noise,

$\boldsymbol{n}=\boldsymbol{w}+\boldsymbol{n}_{\mathrm{c}}$

where the correlated part $(1 / f$ noise) was produced by a stochastic-differential-equation (SDE) method, that produces noise whose power spectrum is approximately of the form $P_{\mathrm{c}}(f) \propto f^{-\alpha}$. It is not of the form $\mathbf{F} \boldsymbol{a}$, but contains a part that cannot be modeled with baselines.

The white noise rms was set to $\sigma=2700 \mu \mathrm{K}$ (thermodynamic scale for CMB anisotropies), corresponding to the PLANCK $70 \mathrm{GHz}$ goal sensitivity (Planck Collaboration 2005). The $1 / f$ noise was simulated with slope $-\alpha=-1.7$ and $f_{\min }=$ $1.15 \times 10^{-5} \mathrm{~Hz}$ (period of one day), so that the power spectrum was flat for $f<f_{\min }$. See Fig. 6. Since the white and $1 / f$ streams were produced separately, the knee frequency $f_{\mathrm{k}}$ (where the white and $1 / f$ noise powers are equal) could be adjusted by multiplying the $1 / f$ stream with different factors. We used $f_{\mathrm{k}}=50 \mathrm{mHz}$ as the reference case, representing a conservative upper limit for the PLANCK $70 \mathrm{GHz}$ detectors (Burigana et al. 1997a; Seiffert et al. 2002; Tuovinen 2003), but consider also $f_{\mathrm{k}}=25 \mathrm{mHz}$ in Sects. 7 and 8 .

The power spectrum of the $1 / f$ noise is thus approximately

$P_{\mathrm{c}}(f)= \begin{cases}\frac{\sigma^{2}}{f_{\mathrm{c}}}\left(\frac{f_{\mathrm{k}}}{f}\right)^{\alpha} & f \geq f_{\min } \\ \frac{\sigma^{2}}{f_{\mathrm{c}}}\left(\frac{f_{\mathrm{k}}}{f_{\min }}\right)^{\alpha} & f \leq f_{\min },\end{cases}$

where $f_{\mathrm{c}}=f_{\text {sample }} / 2=38.4 \mathrm{~Hz}$ is the Nyquist (critical) frequency.

The $1 / f$ noise was generated in $t_{\text {gen }}=15.25 \mathrm{~d}$ pieces. The actual statistics calculated from the simulated $1 / f$ stream were: mean $=-9.86 \mu \mathrm{K}$, stdev $=\sigma_{\mathrm{c}}=1868.5 \mu \mathrm{K}$, so that $\sigma_{\mathrm{c}}^{2}=0.48 \sigma^{2}$. As can be seen from Fig. 6 , the simulated $1 / f$ stream power falls below the noise model for the lowest frequencies. This rounding of the spectrum is a feature of the SDE method (see Keihänen et al. 2009). Although a sizable part of the variance in the $1 / f$ stream comes from the very lowest frequencies, these low frequencies are removed well by destriping, and thus the detailed shape of the spectrum at low frequencies is not that important. For analytical estimates we use the noise model of Eq. (32). 

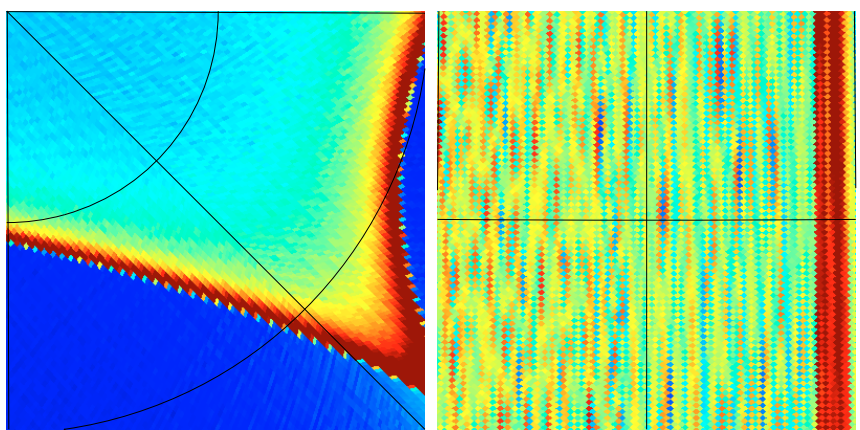

Fig. 7. Hit maps of the two $10^{\circ} \times 10^{\circ}$ regions. The color scale is linear and goes from 0 to 50000 in the left plot, and from 1000 to 3000 in the right plot. The 1-year survey begins and ends near the right edge of the right plot, where the first and last rings overlap to produce a higher hit count.

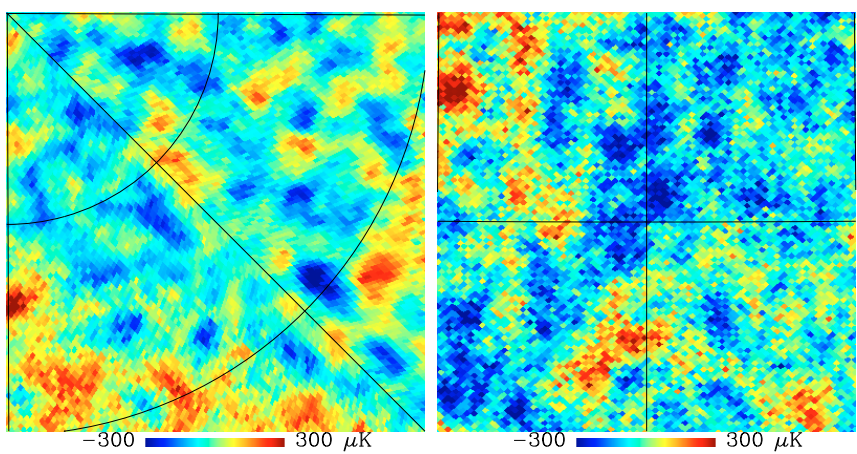

Fig. 8. Destriped (output) temperature map (one year survey, $15 \mathrm{~s}$ baselines) for the two $10^{\circ} \times 10^{\circ}$ regions.

The nonzero mean of the $1 / f$ stream has to be taken into account when comparing solved baselines to the input $1 / f$ stream, since the destriping method sets the average of the solved baselines to zero.

\subsection{Maps}

The maps were produced in the HEALPix ${ }^{3}$ pixelization (Górski et al. 2005) in ecliptic coordinates. We used the $N_{\text {side }}=512$ resolution for all maps, corresponding to $n_{p}=3145728$ for the full sky, with square root of pixel solid angle $\theta_{p} \equiv \Omega_{p}^{1 / 2}=6.87^{\prime}$. For the full-year TOD the polarization of each pixel was well sampled; the lowest rcond was 0.422 . The mean rcond was 0.492 and the maximum 0.49999 . The hit count $n_{\text {hit }}$ (number of hits per pixel) varied from 818 to 273480 for the full 1-year simulation. The mean number of hits was $\left\langle n_{\text {hit }}\right\rangle \equiv n_{t} / n_{p}=3088.125$. The mean inverse hit count was $\left\langle n_{\text {hit }}^{-1}\right\rangle=0.000418=(0.0204)^{2} \approx$ $1 / 2391$. Figure 5 shows the hit count in the regions around the ecliptic poles, where it varies a lot. Figure 7 shows the hit count in the two $10^{\circ} \times 10^{\circ}$ regions.

Figure 8 shows the output temperature map $\boldsymbol{m}_{\text {out }}$ for the case $t_{\text {base }}=15 \mathrm{~s}$. The visual appearance is the same for other baseline lengths. To see differences one has to look at residual maps, see Sect. 6. Polarization maps (Fig. 9) are dominated by small-scale noise. The most obvious map-making related feature in the output maps is the reduction of noise where the hit count is larger. Other effects are more subtle and are analyzed in the following sections.
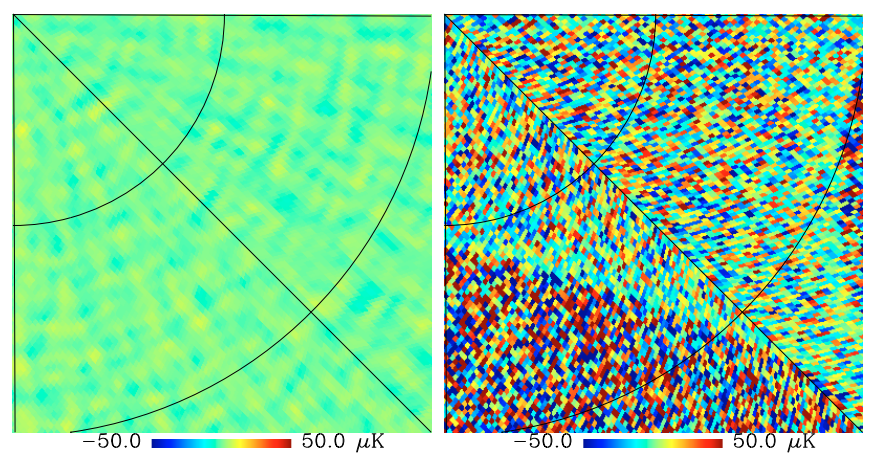

Fig. 9. Even near the ecliptic poles, where the noise in the output map is the lowest, the pixel-scale noise from four $70 \mathrm{GHz}$ detectors is higher than the CMB polarization signal in the map. Left: binned signal Q map. Right: output Q map (15 s baselines). (Both plots are from the same pole region.)

\section{Time domain}

We now analyze the application of the destriping method to this kind of data. We consider the case of the full 1-year survey with $f_{\mathrm{k}}=50 \mathrm{mHz}$ noise, except for Sect. 7, where we discuss the effect of changing the knee frequency, and for Sect. 9, where we consider maps made from shorter pieces of the TOD. Consider this first in the time domain, i.e., look at the cleaned TOD

$\boldsymbol{d} \equiv \boldsymbol{y}-\mathbf{F} \boldsymbol{a}_{\text {out }}$.

We assume that the two noise streams, $\boldsymbol{w}$ and $\boldsymbol{n}_{\mathrm{c}}$ are independent Gaussian random processes.

Since the destriping method is linear, we can divide the baseline amplitudes obtained by Eq. (16) into the parts coming from the different TOD components,

$\boldsymbol{a}_{\mathrm{out}}=\mathbf{A} \boldsymbol{y}=\mathbf{A} s+\mathbf{A} w+\mathbf{A} \boldsymbol{n}_{\mathrm{c}}$

Likewise, the cleaned TOD can be divided into five terms,

$\boldsymbol{d}=\boldsymbol{s}+\boldsymbol{w}-\mathbf{F A} \boldsymbol{w}+\left(\boldsymbol{n}_{\mathrm{c}}-\mathbf{F A} \boldsymbol{n}_{\mathrm{c}}\right)-\mathbf{F A} \boldsymbol{s}$.

The first term is the signal and the second term is the white noise. We call the third term white noise baselines, the fourth term (in parenthesis) residual $1 / f$ noise, and the fifth term signal baselines.

The TOD vector $\mathbf{Z} \boldsymbol{s}$ appearing in the signal baseline term

$\mathbf{F A} \boldsymbol{s} \equiv \mathbf{F D}^{-1} \mathbf{F}^{\mathrm{T}} \mathbf{C}_{w}^{-1} \mathbf{Z} \boldsymbol{s}$

is the pixelization noise (Doré et al. 2001). It is the noise estimate we get from the signal TOD (which contains no noise). Signal gradients (of the beam-smoothed input sky) within a map pixel are the origin of the pixelization noise.

\subsection{Reference baselines}

We define the reference baselines $\mathbf{R} \boldsymbol{n}$ of a noise stream $\boldsymbol{n}$ as the weighted averages of each baseline segment, i.e., matrix $\mathbf{R}$ is defined as

$\mathbf{R} \equiv\left(\mathbf{F}^{\mathrm{T}} \mathbf{C}_{w}^{-1} \mathbf{F}\right)^{-1} \mathbf{F}^{\mathrm{T}} \mathbf{C}_{w}^{-1}$

Note that $\mathbf{R F}=\mathbf{I}$.

The reference baselines of the $1 / f$ noise,

$\boldsymbol{a}_{r} \equiv \mathbf{R} \boldsymbol{n}_{\mathrm{c}}$, 
can be viewed as the "goal" of baseline estimation. Subtracting them from the full TOD gives us a TOD stream

$\boldsymbol{y}-\mathbf{F} \boldsymbol{a}_{r}=\boldsymbol{s}+\boldsymbol{w}+\left(\boldsymbol{n}_{\mathrm{c}}-\mathbf{F} \boldsymbol{a}_{r}\right)$,

which contains, beside the signal and the white noise, only the part $\left(\boldsymbol{n}_{\mathrm{c}}-\mathbf{F} \boldsymbol{a}_{r}\right)$ of correlated noise that cannot be represented in terms of baselines. We call this unmodeled $1 / f$ noise.

The actual cleaned TOD that results from destriping, can now be written as

$\boldsymbol{d}=\boldsymbol{y}-\mathbf{F} \boldsymbol{a}_{\text {out }}=\boldsymbol{s}+\boldsymbol{w}-\mathbf{F A} \boldsymbol{w}+\left(\boldsymbol{n}_{\mathrm{c}}-\mathbf{F} \boldsymbol{a}_{r}\right)-\mathbf{F}\left(\mathbf{A} \boldsymbol{n}_{\mathrm{c}}-\boldsymbol{a}_{r}\right)-\mathbf{F A} \boldsymbol{s}$,

where the residual $1 / f$ noise is split into the unmodeled $1 / f$ noise and the $1 / f$ baseline error, $\mathbf{A} \boldsymbol{n}_{\mathrm{c}}-\boldsymbol{a}_{r}=(\mathbf{A}-\mathbf{R}) \boldsymbol{n}_{\mathrm{c}}$. Thus, altogether, there are three contributions to missing the goal of baseline estimation,

$\boldsymbol{a}_{\mathrm{out}}-\boldsymbol{a}_{r}=\mathbf{A} \boldsymbol{w}+(\mathbf{A}-\mathbf{R}) \boldsymbol{n}_{\mathrm{c}}+\mathbf{A} \boldsymbol{s}$,

white noise baselines, $1 / f$ baseline error, and signal baselines.

Unlike the solved baseline contributions $\mathbf{A} \boldsymbol{n}$, the reference baselines $\mathbf{R} \boldsymbol{n}$ do not involve the pointing matrix (except for the case of pixelization noise), so the differences between them are related to how the scanning strategy connects the baselines with crossing points. Thus, for analyzing errors, it is useful to separate also the white noise baselines into white noise reference baselines and white noise baseline error, $\mathbf{A} w=\mathbf{R} w+(\mathbf{A}-\mathbf{R}) \boldsymbol{w}$; and likewise the signal baselines into reference baselines of pixelization noise and signal baseline error $\mathbf{A} \boldsymbol{s}=\mathbf{R Z} \boldsymbol{s}+(\mathbf{A}-\mathbf{R}) \mathbf{Z} \boldsymbol{s}$.

The white noise baseline error stream $(\mathbf{A}-\mathbf{R}) \boldsymbol{w}$ is uncorrelated with the white noise reference baseline stream $\mathbf{R} w$. To show this, we note that

$$
\begin{aligned}
\mathbf{R C}_{w} \mathbf{A}^{\mathrm{T}} & =\mathbf{R C}_{w}\left(\mathbf{I}-\mathbf{B}^{\mathrm{T}} \mathbf{P}^{\mathrm{T}}\right) \mathbf{C}_{w}^{-1} \mathbf{F} \mathbf{D}^{-1} \\
& =\mathbf{D}^{-1}-\mathbf{R} \mathbf{P B} \mathbf{F} \mathbf{D}^{-1} \\
& =\mathbf{D}^{-1}-\left(\mathbf{F}^{\mathrm{T}} \mathbf{C}_{w}^{-1} \mathbf{F}\right)^{-1}\left(\mathbf{F}^{\mathrm{T}} \mathbf{C}_{w}^{-1} \mathbf{F}-\mathbf{D}\right) \mathbf{D}^{-1} \\
& =\left(\mathbf{F}^{\mathrm{T}} \mathbf{C}_{w}^{-1} \mathbf{F}\right)^{-1}=\mathbf{R} \mathbf{C}_{w} \mathbf{R}^{\mathrm{T}},
\end{aligned}
$$

so that

$$
\left\langle(\mathbf{R} w)((\mathbf{A}-\mathbf{R}) \boldsymbol{w})^{\mathrm{T}}\right\rangle=\mathbf{R} \mathbf{C}_{w} \mathbf{A}^{\mathrm{T}}-\mathbf{R} \mathbf{C}_{w} \mathbf{R}^{\mathrm{T}}=0 .
$$

\subsection{Approximation to solved baselines}

The solved baselines $\boldsymbol{a}_{\text {out }}$ can now be written as

$$
\begin{aligned}
\boldsymbol{a}_{\mathrm{out}}= & \boldsymbol{a}_{r}+\mathbf{A} \boldsymbol{s}+\mathbf{A} \boldsymbol{w}+\mathbf{A}\left(\boldsymbol{n}_{\mathrm{c}}-\mathbf{F} \boldsymbol{a}_{r}\right) \\
= & \boldsymbol{a}_{r}+\left(\mathbf{F}^{\mathrm{T}} \mathbf{C}_{w}^{-1} \mathbf{Z F}\right)^{-1} \mathbf{F}^{\mathrm{T}} \mathbf{C}_{w}^{-1} \mathbf{Z s} \\
& +\left(\mathbf{F}^{\mathrm{T}} \mathbf{C}_{w}^{-1} \mathbf{Z F}\right)^{-1} \mathbf{F}^{\mathrm{T}} \mathbf{C}_{w}^{-1} \mathbf{Z} \boldsymbol{w} \\
& +\left(\mathbf{F}^{\mathrm{T}} \mathbf{C}_{w}^{-1} \mathbf{Z F}\right)^{-1} \mathbf{F}^{\mathrm{T}} \mathbf{C}_{w}^{-1} \mathbf{Z}\left(\boldsymbol{n}_{\mathrm{c}}-\mathbf{F} \boldsymbol{a}_{r}\right)
\end{aligned}
$$

(up to an overall constant), so that

$$
\begin{aligned}
\mathbf{F}^{\mathrm{T}} \mathbf{C}_{w}^{-1} \mathbf{Z F}\left(\boldsymbol{a}_{\text {out }}-\boldsymbol{a}_{r}\right)= & \mathbf{F}^{\mathrm{T}} \mathbf{C}_{w}^{-1} \mathbf{Z} \boldsymbol{s}+\mathbf{F}^{\mathrm{T}} \mathbf{C}_{w}^{-1} \mathbf{Z} \boldsymbol{w} \\
& +\mathbf{F}^{\mathrm{T}} \mathbf{C}_{w}^{-1} \mathbf{Z}\left(\boldsymbol{n}_{\mathrm{c}}-\mathbf{F} \boldsymbol{a}_{r}\right) .
\end{aligned}
$$

For the TOD streams $\mathbf{F}\left(\boldsymbol{a}_{\text {out }}-\boldsymbol{a}_{r}\right), \boldsymbol{w}$, and $\boldsymbol{n}_{\mathrm{c}}-\mathbf{F} \boldsymbol{a}_{r}$ there should be no significant correlations between the samples from different circles that hit the same pixel. (If the baseline length is longer than one scanning circle, this statement is limited to the samples that come from different baseline segments for $\mathbf{F}\left(\boldsymbol{a}_{\text {out }}-\boldsymbol{a}_{r}\right)$ and $\boldsymbol{n}_{\mathrm{c}}-\mathbf{F} \boldsymbol{a}_{r}$.) Therefore, for a large number of hits, the effect of the
$\mathbf{P B}$ part of $\mathbf{Z}$ tends to average out, leaving the $\mathbf{I}$ part dominant, and we can approximate $\mathbf{Z} \approx \mathbf{I}$, leading to $\mathbf{A} \approx \mathbf{R}$, for these terms. We get the approximation

$\boldsymbol{a}_{\mathrm{out}} \approx \boldsymbol{a}_{r}+\mathbf{R} \boldsymbol{w}+\mathbf{R Z} \boldsymbol{s}$.

Comparing to Eq. (41), we note that in Eq. (46) white noise baselines are approximated by white noise reference baselines, $1 / f$ baseline error is ignored, and signal baselines are approximated by the reference baselines of pixelization noise. Thus, for a good scanning, the solved baselines $\boldsymbol{a}_{\text {out }}$ should track the reference baselines of instrument noise + pixelization noise.

\subsection{Division}

The effect of destriping on the white noise is just harmful for the maps (we elaborate on this in Sect. 6), so the relevant time domain residual is

$$
\begin{aligned}
\boldsymbol{d}-\boldsymbol{s}-\boldsymbol{w} & =\boldsymbol{n}_{\mathrm{c}}-\mathbf{F} \boldsymbol{a}_{\text {out }} \\
& =-\mathbf{F A} \boldsymbol{w}+\left(\boldsymbol{n}_{\mathrm{c}}-\mathbf{F A} \boldsymbol{n}_{\mathrm{c}}\right)-\mathbf{F A} \boldsymbol{s} .
\end{aligned}
$$

It consists of three components:

1. white noise baselines;

2. residual $1 / f$ noise;

3. and signal baselines;

which are uncorrelated with each other.

Each component can be further divided into two parts:

$$
\begin{aligned}
\boldsymbol{d}-\boldsymbol{s}-\boldsymbol{w}= & -\mathbf{F R} \boldsymbol{w}-\mathbf{F}(\mathbf{A}-\mathbf{R}) \boldsymbol{w} \\
& +\left(\boldsymbol{n}_{\mathrm{c}}-\mathbf{F} \boldsymbol{a}_{r}\right)-\mathbf{F}\left(\mathbf{A} \boldsymbol{n}_{\mathrm{c}}-\boldsymbol{a}_{r}\right) \\
& -\mathbf{F R Z} \boldsymbol{s}-\mathbf{F}(\mathbf{A}-\mathbf{R Z}) \boldsymbol{s} .
\end{aligned}
$$

We call these six components:

$1 \alpha$ ) white noise reference baselines;

$1 \beta$ ) white noise baseline error;

$2 \alpha$ ) unmodeled $1 / f$ noise;

$2 \beta) 1 / f$ baseline error;

$3 \alpha$ ) reference baselines of pixelization noise;

$3 \beta$ ) signal baseline error.

Of these components, $1 \alpha, 1 \beta, 2$ and 3 are uncorrelated with each other. This division forms the basis of the discussion in the rest of this paper. Approximation (46) corresponds to ignoring the $\beta$-components. Components $1 \alpha$ and $2 \alpha$ are independent of scanning strategy, which makes their properties easier to understand. The $\beta$-components are related to how the baselines are solved using crossing points and thus couple to the scanning strategy in a complicated manner.

We turn now to our results with simulated data to see how this comes out in practice, first for the noise part, and then for the signal part.

In Fig. 10 we show a part of the simulated $(1 / f+$ white $)$ noise stream $\boldsymbol{n}$, both the reference and solved baselines, FR $\boldsymbol{n}$ and $\mathbf{F A} \boldsymbol{n}$, and their difference $\mathbf{F}(\mathbf{R}-\mathbf{A}) \boldsymbol{n}=\mathbf{F}(\mathbf{A}-\mathbf{R}) \boldsymbol{n}_{\mathrm{c}}+\mathbf{F}(\mathbf{A}-\mathbf{R}) \boldsymbol{w}$, for $t_{\text {base }}=15 \mathrm{~s}$.

We consider now separately the white noise and $1 / f$ noise parts.

\subsection{White noise baselines}

From Tables 2 and 3 we see that the white noise baselines track their reference baselines well. The white noise reference baselines are just white noise themselves, their variance $\sigma_{w r}^{2}$ down 


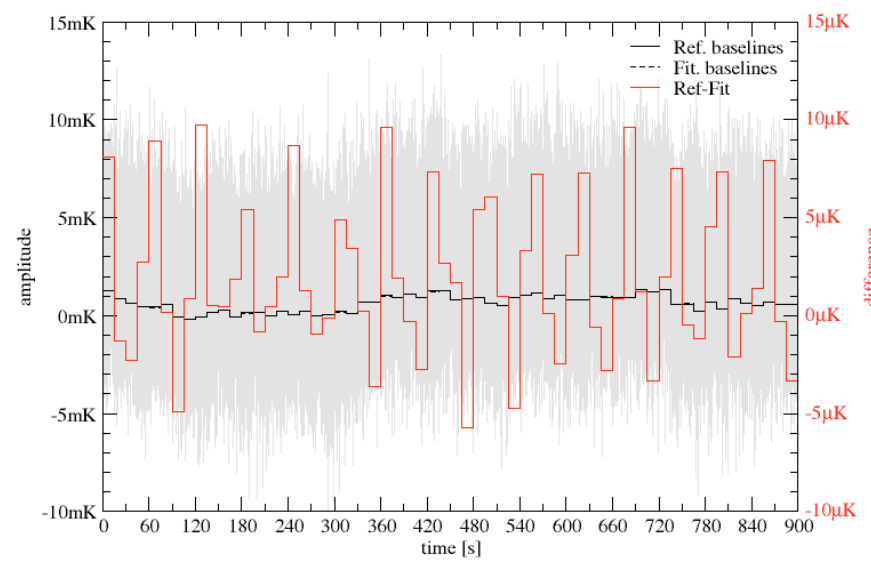

Fig. 10. First 15 minutes of the $(1 / f+$ white) noise stream (grey) and its reference (black solid) and solved (black dashed) $t_{\text {base }}=15 \mathrm{~s}$ baselines. Note that these two baseline curves are nearly on top of each other. The difference of the curves is plotted as the red curve and corresponds to the scale on the right. This represents the error in the approximation (46), except for the signal contribution to it.

Table 2. Statistics of the white noise baselines.

\begin{tabular}{cccc}
\hline \hline$t_{\text {base }}$ & $\begin{array}{c}\text { Eq. }(29) \\
(\mu \mathrm{K})\end{array}$ & $\begin{array}{c}\sigma_{w r} \text { (reference) } \\
(\mu \mathrm{K})\end{array}$ & $\begin{array}{c}\sigma_{w b} \text { (solved) } \\
(\mu \mathrm{K})\end{array}$ \\
\hline $2.5 \mathrm{~s}$ & 194.856 & 194.845 & 195.115 \\
$15 \mathrm{~s}$ & 79.550 & 79.551 & 79.631 \\
$1 \mathrm{~min}$ & 39.775 & 39.766 & 39.808 \\
$1 \mathrm{~h}$ & 5.135 & 5.130 & 5.399 \\
\hline
\end{tabular}

Table 3. Stdev $\sigma_{w e}$ of white noise baseline error.

\begin{tabular}{cccc}
\hline \hline$t_{\text {base }}$ & $\begin{array}{c}a \text { and } b \\
(\mu \mathrm{K})\end{array}$ & $\begin{array}{c}(a+b) / 2 \\
(\mu \mathrm{K})\end{array}$ & $\begin{array}{c}(a-b) / 2 \\
(\mu \mathrm{K})\end{array}$ \\
\hline $2.5 \mathrm{~s}$ & 10.244 & 5.916 & 8.354 \\
$15 \mathrm{~s}$ & 3.595 & 2.063 & 2.944 \\
$1 \mathrm{~min}$ & 1.854 & 1.066 & 1.517 \\
$1 \mathrm{~h}$ & 1.719 & 0.989 & 1.406 \\
\hline
\end{tabular}

from the white noise variance $\sigma^{2}$ by $n_{\text {base. }}$ The white noise baseline variance

$\sigma_{w b}^{2} \approx \sigma_{w r}^{2}=\frac{\sigma^{2}}{n_{\text {base }}}=\left(\frac{f_{x}}{f_{\mathrm{c}}}\right) \sigma^{2}=\frac{\sigma^{2}}{f_{s} t_{\text {base }}}$,

is slightly larger. Table 2 shows the standard deviation (square root of the variance) of the baselines, $\sigma_{w r}$ and $\sigma_{w b}$.

In Table 3 we give the standard deviation $\sigma_{w e}$ of the white noise baseline error. We show it also for the average and the difference between the two polarization directions $a$ and $b$, which represent the contribution of this effect to the temperature and polarization measurements.

The white noise reference baselines are completely uncorrelated with each other. This is not true for the solved white noise baselines. The difference, the baseline errors, show significant autocorrelation, see Figs. 11 and 12, and correlation between detectors 19 and 22, see Table 4. Although the white noise baseline variance is not much larger than the white noise reference baseline variance, these correlations make the difference between them important.

These correlation properties are easy to understand. While the amplitude of the reference baseline arises from the noise of the baseline segment itself, the error $(\mathbf{A}-\mathbf{R}) \boldsymbol{w}$ is caused by the noise in the crossing baselines. Since the baseline segments that

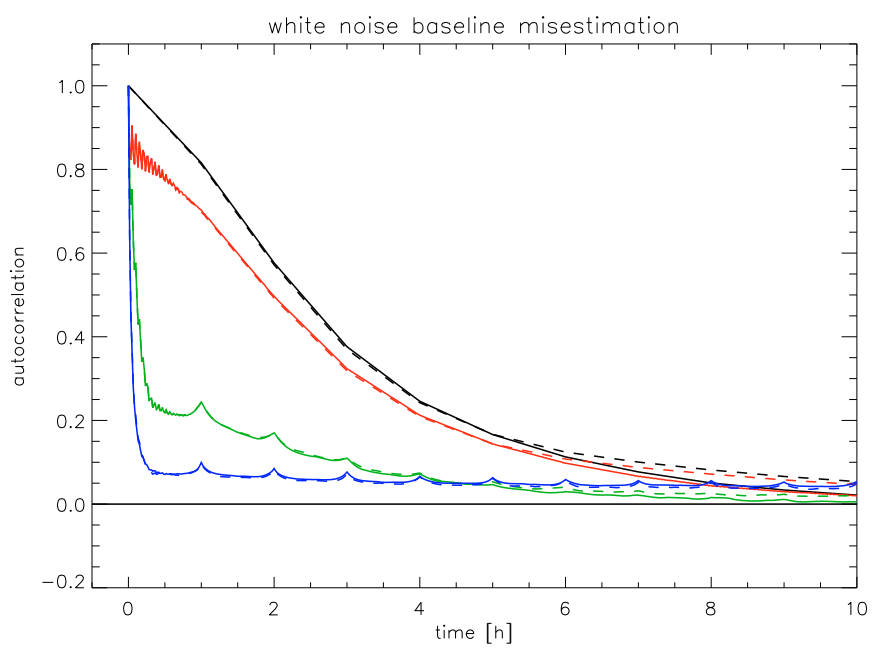

Fig. 11. Autocorrelation function for the white noise baseline error $(\mathbf{A}-\mathbf{R}) w$ for $t_{\text {base }}=1 \mathrm{~h}$ (black), $1 \mathrm{~min}$ (red), $15 \mathrm{~s}$ (green), $2.5 \mathrm{~s}$ (blue). The solid lines are for the "temperature" baselines $(a+b) / 2$ and the dashed lines are for the "polarization" baselines $(a-b) / 2$. For the $t_{\text {base }}=15 \mathrm{~s}$ and $2.5 \mathrm{~s}$ cases, only lags that are multiples of $1 \mathrm{~min}$ $(\approx$ the spin period) are included.

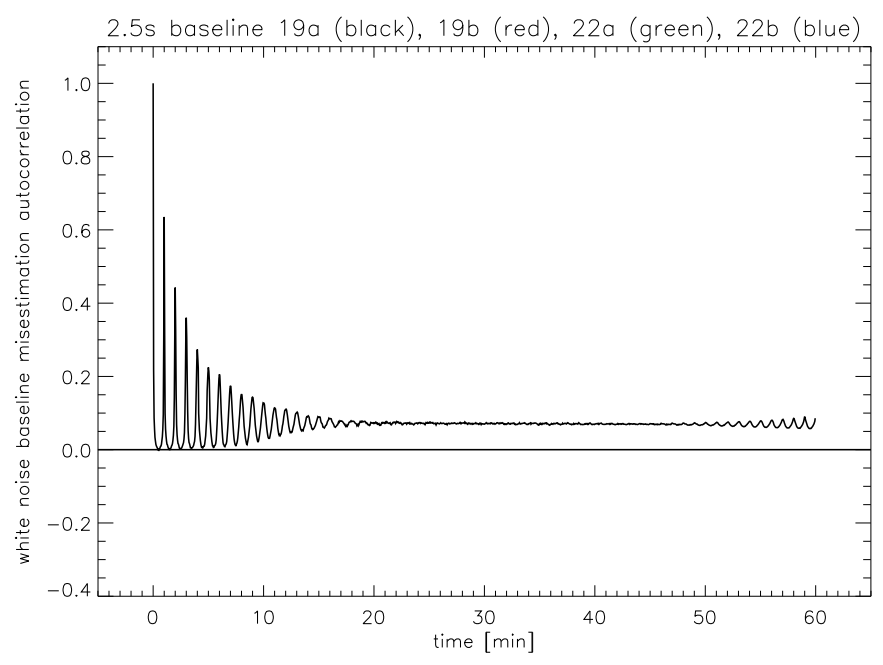

Fig. 12. Autocorrelation function for the white noise baseline error $(\mathbf{A}-\mathbf{R}) \boldsymbol{w}$ for $t_{\text {base }}=2.5 \mathrm{~s}$ and detector $19 \mathrm{a}$.

Table 4. Correlations between detectors 19 and 22.

\begin{tabular}{cccc}
\hline \hline$t_{\text {base }}$ & $(a+b) / 2$ & $(a-b) / 2$ & Overlap \\
\hline $2.5 \mathrm{~s}$ & 0.826 & -0.003 & 0.793 \\
$15 \mathrm{~s}$ & 0.901 & 0.005 & 0.966 \\
$1 \mathrm{~min}$ & 0.925 & -0.011 & 0.991 \\
$1 \mathrm{~h}$ & 0.998 & -0.013 & 0.99986 \\
\hline
\end{tabular}

are separated from each other by an integer number of spin periods, and not too many pointing periods, cross almost the same set of other baseline segments, often in the same pixels, their baseline errors $(\mathbf{A}-\mathbf{R}) \boldsymbol{w}$ are strongly correlated with each other.

In Fig. 12 we show the autocorrelation function of $(\mathbf{A}-\mathbf{R}) \boldsymbol{w}$ for the case $t_{\text {base }}=2.5 \mathrm{~s}$. For short lags, only baselines whose lag is a multiple of $1 \mathrm{~min}(\approx$ the spin period) show significant correlation with each other. For longer lags this distinction disappears due to random spin rate variations. Since these variations have a rms which is about $1 / 60$ of the spin rate, for lags around 30 min any one of the baseline segments within a spin period 


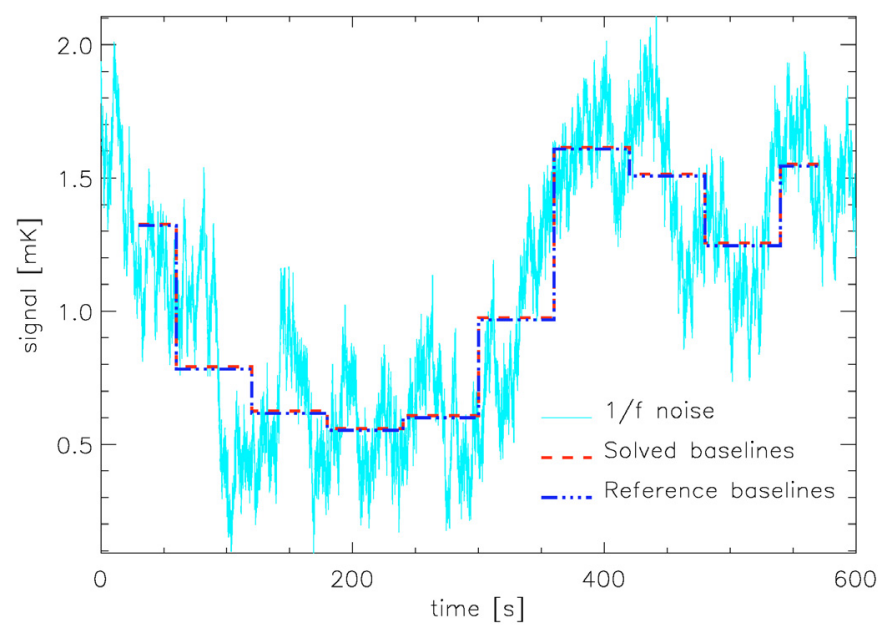

Fig. 13. First $10 \mathrm{~min}$ of the $1 / f$ noise stream $\boldsymbol{n}_{\mathrm{c}}$ together with its solved and reference baselines for $t_{\text {base }}=1 \mathrm{~min}$. The difference between the $1 / f$ noise and its reference baselines is the unmodeled $1 / f$ noise.

is about equally likely to land on a given location of the scan circle. The correlation between two baselines that land on the same location of the circle is much larger (presumably similar to the $t_{\text {base }}=1 \mathrm{~min}$ and $1 \mathrm{~h}$ cases), but the way we calculate the autocorrelation function (in time domain, not in the spin phase domain) is not able to pick this out.

Also, since the corresponding baseline segments from the horns 19 and 22 are only $\omega_{\text {scan }} 3.1^{\circ}=0.517 \mathrm{~s}$ shifted from each other, they crossed almost the same set of other baseline segments, and are thus strongly correlated. The overlap fraction $1-0.517 \mathrm{~s} / t_{\text {base }}$ is given in Table 4 . Note that this number does not take into account that the hits from the two horns may still be distributed differently to the pixels in the overlap region.

More exactly, the "temperature" combinations $(a+b) / 2$ of $(\mathbf{A}-\mathbf{R}) \boldsymbol{w}$ are strongly correlated between 19 and 22, whereas the "polarization" combinations $(a-b) / 2$ are not. The polarization directions of the $a b$ pairs 19 and 22 differ from each other by $44.8^{\circ}$ making their polarization measurements $(a-b) / 2$ almost orthogonal. Thus they also pick almost orthogonal error combinations from the crossing baseline segments, and remain uncorrelated. This also explains why the stdev of the "polarization baseline error" is larger than the "temperature" one in Table 3. In effect, only half of the crossing baseline pairs $a b$ contribute to determining an $(a-b) / 2$ baseline combination, so the number of degrees of freedom is down by $1 / 2$ and the variance thus larger by 2 .

\subsection{Residual $1 /$ f noise}

Figure 13 shows the $1 / f$ part of the noise $\boldsymbol{n}_{\mathrm{c}}$, its reference baselines $\boldsymbol{a}_{r}$ and the solved $1 / f$ baselines, $\mathbf{A} \boldsymbol{n}_{\mathrm{c}}$. The difference between these two sets of baselines, $1 / f$ baseline error, is shown in Fig. 14.

The $1 / f$ noise can be separated into reference baselines and unmodeled $1 / f$ noise, $\boldsymbol{n}_{\mathrm{c}}=\mathbf{F} \boldsymbol{a}_{r}+\left(\boldsymbol{n}_{\mathrm{c}}-\mathbf{F} \boldsymbol{a}_{r}\right)$. When we separate the $1 / f$ baseline error, $(\mathbf{A}-\mathbf{R}) \boldsymbol{n}_{\mathrm{c}}$ into the corresponding components,

$$
(\mathbf{A}-\mathbf{R}) \boldsymbol{n}_{\mathrm{c}}=(\mathbf{A}-\mathbf{R}) \mathbf{F} \boldsymbol{a}_{r}+\mathbf{A}\left(\boldsymbol{n}_{\mathrm{c}}-\mathbf{F} \boldsymbol{a}_{r}\right),
$$

we note that the first term on the right hand side gives the same contribution to each baseline ( $=-$ the mean of the $1 / f$ noise), and is thus irrelevant. Thus the $1 / f$ baseline error arises from
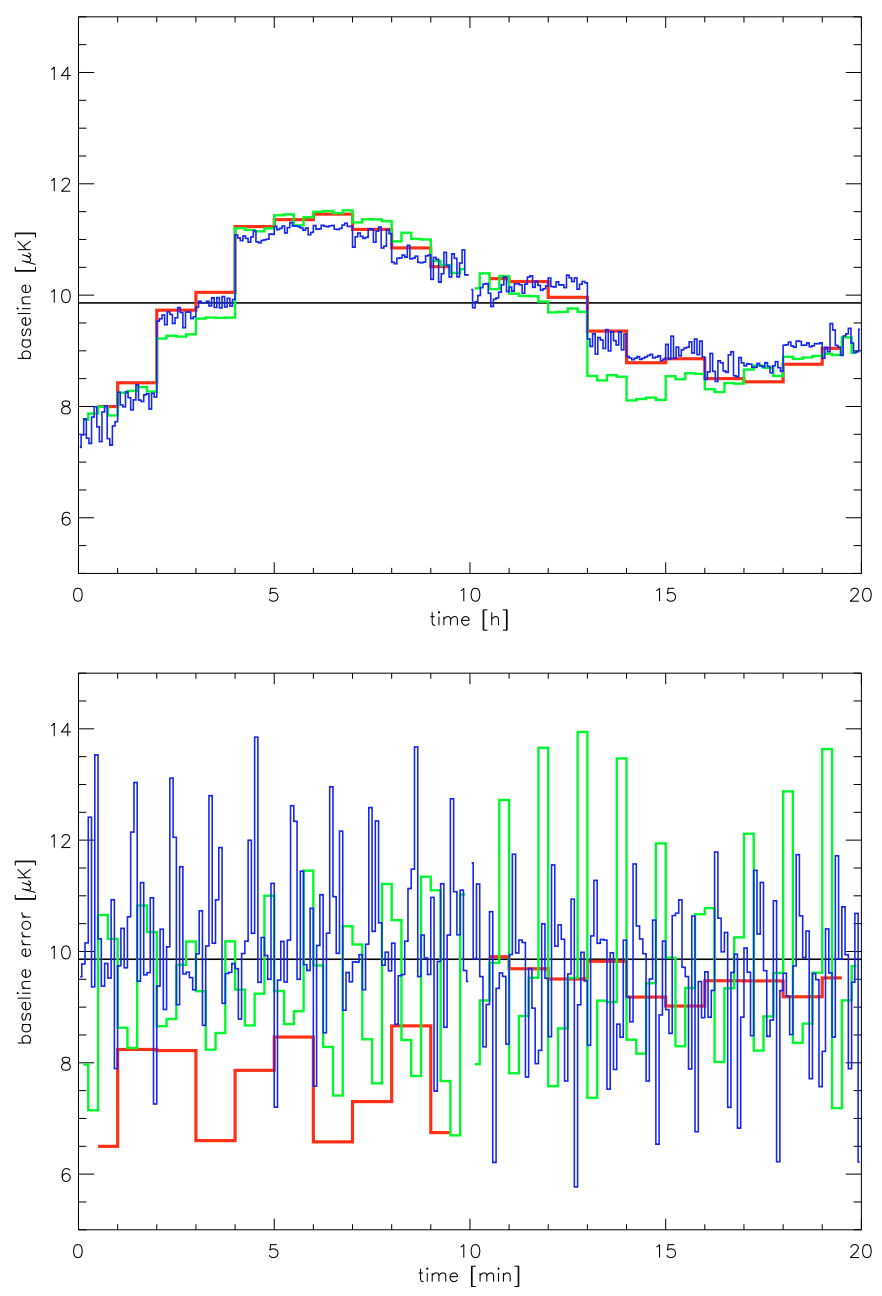

Fig. 14. The $1 / f$ baseline error, i.e., the difference between the solved and reference $1 / f$ baselines $\mathbf{F A} \boldsymbol{n}_{\mathrm{c}}-\mathbf{F} \boldsymbol{a}_{r}$. The top panel shows the case for $t_{\text {base }}=1 \mathrm{~h}$ (red), $15 \mathrm{~min}$ (green), and $4 \mathrm{~min}$ (blue) from the first $10 \mathrm{~h}$ and from another set of $10 \mathrm{~h}$ from the second month of the simulation. The bottom panel shows the case for $t_{\text {base }}=1 \min ($ red $), 15 \mathrm{~s}$ (green), and $5 \mathrm{~s}$ (blue) from the first $10 \mathrm{~min}$ and from another set of 10 min from the second month of the simulation. The horizontal line at $9.86 \mu \mathrm{K}$ shows the mean difference that is due to the nonzero mean of the $1 / f$ noise. Only the deviations from this mean are significant.

the unmodeled $1 / f$ noise, in the same manner as white noise baseline error $(\mathbf{A}-\mathbf{R}) \boldsymbol{w}$ arises from white noise. (The reference baselines of unmodeled $1 / f$ noise are zero).

\subsubsection{Unmodeled $1 / f$ noise}

Since baselines can only model frequencies $\leq f_{x} \equiv 1 /\left(2 t_{\text {base }}\right)$ and do it better for lower frequencies, the power spectrum of the unmodeled $1 / f$ noise $\boldsymbol{n}_{\mathrm{c}}-\mathbf{F} \boldsymbol{a}_{r}$ is equal to that of $\boldsymbol{n}_{\mathrm{c}}$ for $f \gg f_{x}$ and falls rapidly towards smaller $f$ for $f<f_{x}$. See Fig. 15 .

Since the reference baselines $\boldsymbol{a}_{r}$ are obtained from the stream $\boldsymbol{n}_{\mathrm{c}}$, the power spectrum $P_{\mathrm{u}}(f)$ of the unmodeled $1 / f$ noise $\boldsymbol{n}_{\mathrm{c}}-\mathbf{F} \boldsymbol{a}_{r}$ can be obtained from the power spectrum $P_{\mathrm{c}}(f)$ of $\boldsymbol{n}_{\mathrm{c}}$ through a transfer function,

$P_{\mathrm{u}}(f)=H(f) P_{\mathrm{c}}(f)$.

This transfer function is

$H(f)=\left[1-\frac{\sin ^{2}\left(\pi f t_{\text {base }}\right)}{\left(\pi f t_{\text {base }}\right)^{2}}\right]^{2}$ 


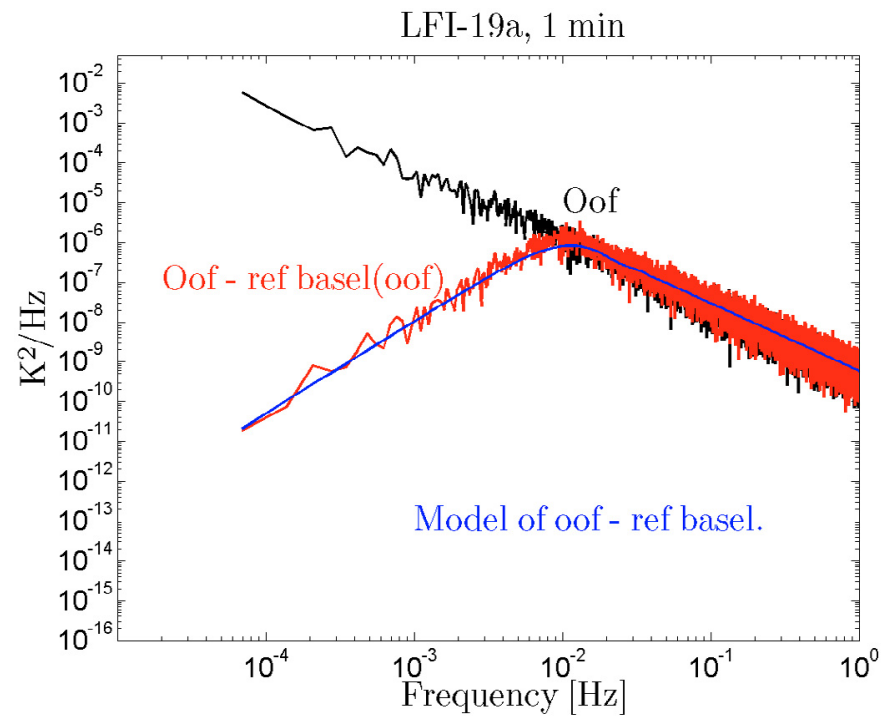

Fig. 15. Power spectrum $P_{\mathrm{c}}(f)$ of the $1 / f$ noise (black) and its unmodeled part $P_{\mathrm{u}}(f)($ red $)$, for $t_{\text {base }}=1 \mathrm{~min}$. The blue line is an analytical estimate corresponding to Eqs. (32), (51), and (52).

Table 5. Stdev $\sigma_{u}$ of the unmodeled $1 / f$ Stream.

\begin{tabular}{cccc}
\hline \hline$t_{\text {base }}$ & $\begin{array}{c}\text { Eq. (56) } \\
(\mu \mathrm{K})\end{array}$ & $\begin{array}{c}\text { Eq. (51) } \\
(\mu \mathrm{K})\end{array}$ & $\begin{array}{c}\text { Actual } \\
(\mu \mathrm{K})\end{array}$ \\
\hline $7.5 \mathrm{~s}$ & 115.9 & 103.1 & 121.7 \\
$15 \mathrm{~s}$ & 147.8 & 131.7 & 154.1 \\
$1 \mathrm{~min}$ & 240.0 & 214.4 & 250.3 \\
$1 \mathrm{~h}$ & 1006.0 & 897.3 & 938.0 \\
\hline
\end{tabular}

For $f \ll f_{x}, H(f) \propto f^{4}$, so the slope of $P_{\mathrm{u}}(f)$ is $4-\alpha$ for $f \ll f_{x}$.

We get a rough estimate of the total power in $\boldsymbol{n}_{\mathrm{c}}-\mathbf{F} \boldsymbol{a}_{r}$ by integrating the original $P_{\mathrm{c}}(f)$ from $f_{x}$ to $f_{\mathrm{c}}$ and $\left(f / f_{x}\right)^{4} P_{\mathrm{c}}(f)$ from $f_{\min }$ to $f_{x}$,

$$
\begin{aligned}
\sigma_{u}^{2} & \approx \int_{f_{\min }}^{f_{x}}\left(\frac{f}{f_{x}}\right)^{4} P_{\mathrm{c}}(f) \mathrm{d} f+\int_{f_{x}}^{f_{\mathrm{c}}} P_{\mathrm{c}}(f) \mathrm{d} f \\
& \approx\left\{\frac{1}{5-\alpha}+\frac{1}{\alpha-1}\left[1-\left(\frac{f_{x}}{f_{\mathrm{c}}}\right)^{\alpha-1}\right]\right\} \sigma^{2} \frac{f_{\mathrm{k}}}{f_{\mathrm{c}}}\left(\frac{f_{\mathrm{k}}}{f_{x}}\right)^{\alpha-1} \\
& =\sigma^{2}\left(\frac{f_{\mathrm{k}}}{f_{\mathrm{c}}}\right)^{\alpha}\left\{\frac{1}{5-\alpha}\left(\frac{f_{\mathrm{c}}}{f_{x}}\right)^{\alpha-1}+\frac{1}{\alpha-1}\left[\left(\frac{f_{\mathrm{c}}}{f_{x}}\right)^{\alpha-1}-1\right]\right\} .
\end{aligned}
$$

For $\alpha=1$ this becomes

$\sigma_{u}^{2} \approx \sigma^{2}\left(\frac{f_{\mathrm{k}}}{f_{\mathrm{c}}}\right)\left[\frac{1}{4}+\ln \frac{f_{\mathrm{c}}}{f_{\mathrm{k}}}\right]$.

For our case, $\alpha=1.7, \sigma=2700 \mu \mathrm{K}$, and we can approximate

$1-\left(\frac{f_{x}}{f_{\mathrm{c}}}\right)^{\alpha-1} \approx 1$,

so that Eq. (53) gives

$\sigma_{u} \approx 57.3 \mu \mathrm{K} t_{\text {base }}(\mathrm{s})^{0.35} \propto t_{\text {base }}^{(\alpha-1) / 2}$.

In Table 5 we compare this approximation to a numerical integration of Eq. (51) and the actual standard deviation $\sigma_{u}$ of $\boldsymbol{n}_{\mathrm{c}}-\mathbf{F} \boldsymbol{a}_{r}$ from our simulated data. The last column is $\sigma_{u}$ calculated from the first $16 \mathrm{~h}$ of the simulated LFI-19a $1 / f$ stream. We see that Eq. (56) is an underestimate. An exception to this is the 1-h-baseline case; this is explained by the simulated noise having less power at low frequencies than the noise model.
Table 6. Stdev $\sigma_{u b}$ of the $1 / f$ Baseline Error.

\begin{tabular}{cccc}
\hline \hline$t_{\text {base }}$ & $\begin{array}{c}a \text { and } b \\
(\mu \mathrm{K})\end{array}$ & $\begin{array}{c}(a+b) / 2 \\
(\mu \mathrm{K})\end{array}$ & $\begin{array}{c}(a-b) / 2 \\
(\mu \mathrm{K})\end{array}$ \\
\hline $5 \mathrm{~s}$ & 1.790 & 0.995 & 1.486 \\
$15 \mathrm{~s}$ & 1.894 & 1.075 & 1.557 \\
$1 \mathrm{~min}$ & 1.404 & 0.765 & 1.177 \\
$1 \mathrm{~h}$ & 1.360 & 0.726 & 1.142 \\
\hline
\end{tabular}

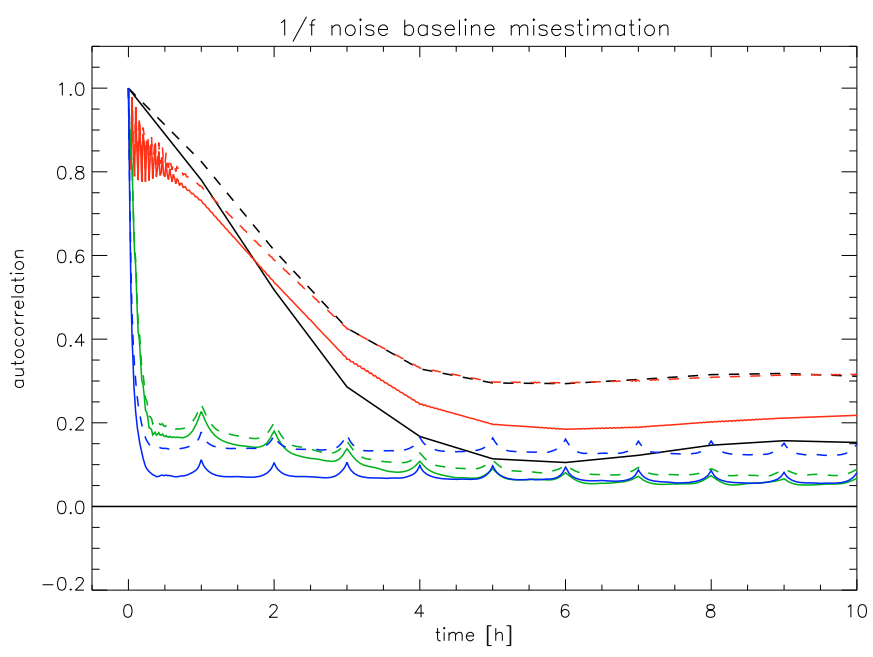

Fig. 16. Same as Fig. 11, but now for the $1 / f$ noise baseline error.

\subsection{2. $1 / f$ baseline error}

Since the unmodeled $1 / f$ noise is correlated, the properties of $1 / f$ baseline errors differ from white noise baseline errors. Over timescales $\ll t_{\text {base }}$ the unmodeled $1 / f$ is positively correlated, but since it averages to zero over each baseline segment, there is a net anticorrelation.

From Fig. 14 we see for short baselines a spin-synchronous pattern. For baselines of $t_{\text {base }}=1 \mathrm{~min}$, the remaining pattern shows a 3-min period that comes from spin-axis nutation. For even longer baselines the long-time-scale correlation shows up clearly. $1 / f$ baseline error decreases for longer baselines, but much less steeply than white noise baseline error. See Table 6, where we show the standard deviation $\sigma_{u b}$ of the $1 / f$ baseline error $\mathbf{A} \boldsymbol{n}_{\mathrm{c}}-\mathbf{R} \boldsymbol{n}_{\mathrm{c}}$.

Figure 16 shows the $1 / f$ baseline error autocorrelation. For white noise baseline error we noted that we get a correlation for nearby baselines since they often cross the same other baseline in the same pixel. Since unmodeled $1 / f$ noise is positively correlated over short timescales, it is not necessary for this crossing to occur in the same pixel to get the positive correlation. Therefore we now get positive correlations for even longer timescales. This makes $1 / f$ baseline error more important than its small variance suggests, since it is not averaged away when binned onto the map.

In Fig. 17 we show the $1 / f$ baseline error for $t_{\text {base }}=1 \mathrm{~h}$ over the full year of the simulation for the detector pair 19. The effect of the 6-month period of the cycloidal scanning is clearly visible.

\subsection{Noise power spectra}

We show the power spectra of the cleaned (destriped) noise streams $\boldsymbol{n}-\mathbf{F A} \boldsymbol{n}$ for different baseline lengths in Fig. 18. They are 


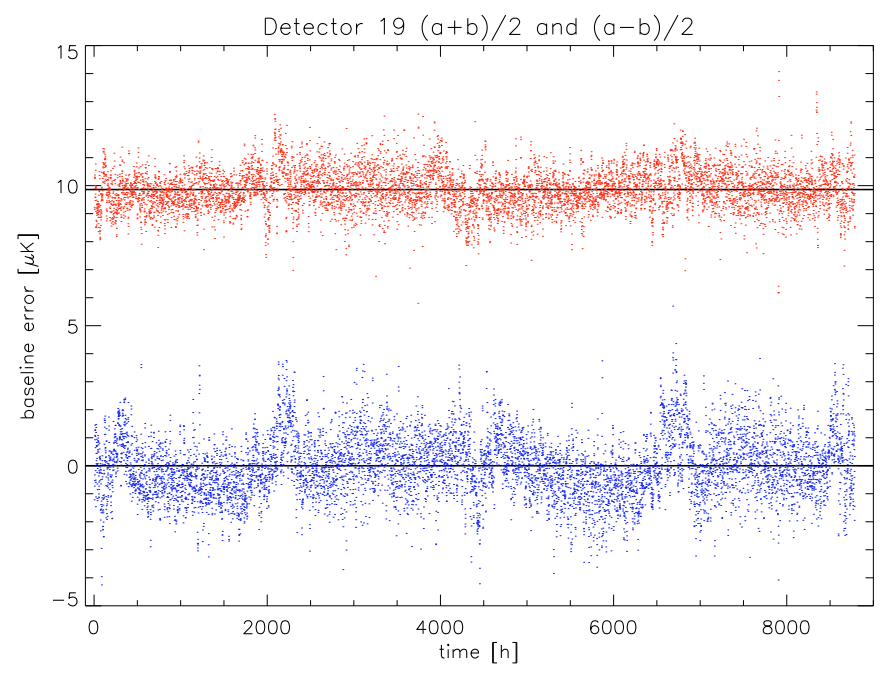

Fig. 17. The $1 / f$ baseline error, $\mathbf{F A} \boldsymbol{n}_{\mathrm{c}}-\mathbf{F} \boldsymbol{a}_{r}$, for 1-h baselines for the full 1-year simulation. We show the sum (red) and the difference (blue) between the two polarization directions. The times when ring crossings occur near the corners of the caustics around the ecliptic poles are centered roughly around hours 240,1950, 4610, and 6340. Correlations in baseline amplitudes are enhanced near these times.

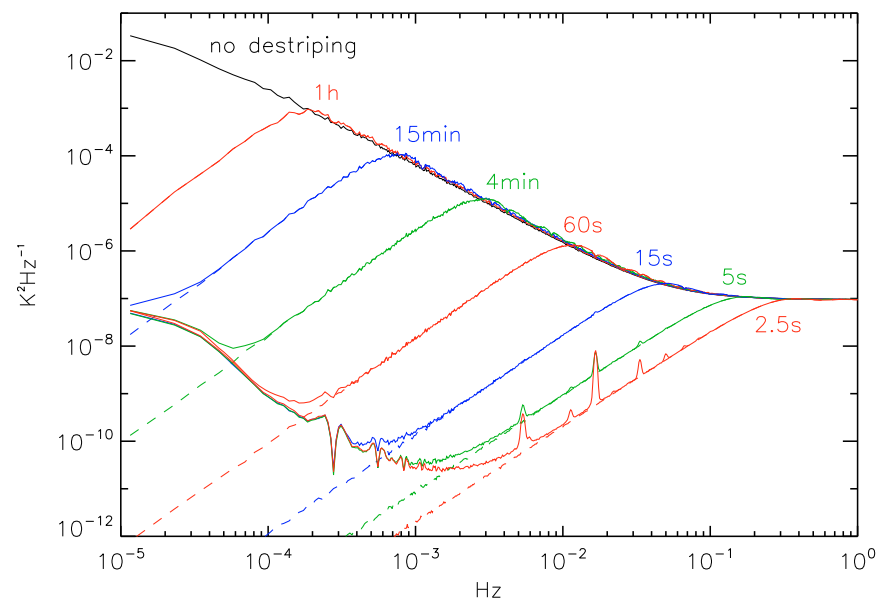

Fig. 18. Effect of baseline subtraction on the noise power spectrum. The solid black line is the spectrum of the original noise stream $\boldsymbol{n}$. The $\mathrm{col}$ ored lines show the spectra of cleaned noise streams for different baseline lengths. Solid lines are for the case of subtracting the solved baselines, $\boldsymbol{n}-\mathbf{F A} \boldsymbol{n}$, and the dashed lines for subtracting reference baselines, $n-\mathbf{F R} n$.

compared to the spectrum of the original noise stream $\boldsymbol{n}$ and the spectra where noise reference baselines are subtracted instead, $\boldsymbol{n}-\mathbf{F R} n$.

Subtraction of baselines suppresses noise at $f \lesssim 1 / t_{\text {base }}$. For lower frequencies the noise is suppressed more, as baselines can model the lower frequencies better. When reference baselines are subtracted, the noise power keeps going down toward lower frequencies; however the solved baselines seem to be able to suppress noise power about 6 orders of magnitude only.

For shorter baselines, spectral features appear at special frequencies. They do not appear when reference baselines are subtracted, so they are clearly related to the scanning strategy. 1) There are peaks at $f=1 / \mathrm{min}$ and $f=1 /(3 \mathrm{~min})$ corresponding to the spin and nutation frequencies, and their harmonics.

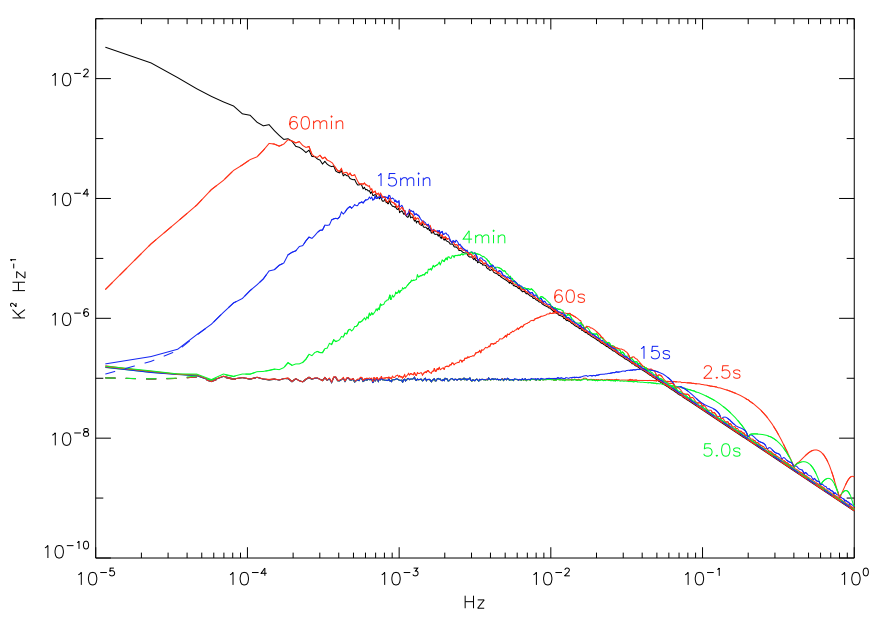

Fig. 19. Effect of subtracting both the white noise and the $1 / f$ baselines on the power spectrum of the $1 / f$ noise. The solid black line is the original $1 / f$ spectrum. The solid colored lines show the power spectra of $\boldsymbol{n}_{\mathrm{c}}-\mathbf{F A} \boldsymbol{n}$ and the dashes lines the power spectra of $\boldsymbol{n}_{\mathrm{c}}-\mathbf{F R} \boldsymbol{n}$.

2) There is a notch in power at $f=1 / \mathrm{h}$, corresponding to the repointing period, and its harmonics. These are easy to understand:

The solved baselines come from a noise estimate based on subtracting from each sample the average of all samples that hit the same pixel. Consider an ideal scanning where the same pixel sequence is hit during each spin period within a repointing period:

1) If the spin period is equal to or a multiple of the period of a noise frequency component, all samples hitting the same pixel during a given repointing period get the same value from this noise component. Thus this noise component can be detected as noise (and not signal) only by comparing hits from different repointing periods, resulting in a much poorer noise estimate.

2) On the other hand, if the repointing period is equal to or a multiple of the noise period, but the spin period is not, then the different samples hitting the same pixel average to zero. This noise component is then recognized as noise in its entirety, and the solved baseline becomes equal to the reference baseline.

Since in our simulation the scanning deviated from ideal, these features are weakened, but still clearly visible.

As mentioned in Sects. 1 and 5.3 and elaborated in Sect. 6, the relevant residual noise is the stream $\boldsymbol{n}_{\mathrm{c}}-\mathbf{F A} \boldsymbol{n}$ where both white noise and $1 / f$ baselines are subtracted from the $1 / f$ noise stream. This is shown in Fig. 19. The subtraction of the white noise baselines has added power to the cleaned $1 / f$ stream. At low frequencies the spectrum appears now white, since the white noise reference baseline stream is white at timescales longer than $t_{\text {base }}$. The power of the baseline error rises towards the lowest frequencies and shows up below $5 \times 10^{-5} \mathrm{~Hz}$. The subtraction of uniform baselines from the noise stream has a chopping effect that transforms a part of the low frequency, $f<1 / t_{\text {base }}$, power into high frequency, $f \gtrsim 1 / t_{\text {base }}$.

\subsection{Optimal baseline length}

What is the optimal baseline length to use? A partial answer can be found by minimizing the variance of the residual correlated noise, i.e., the residual noise minus the original white noise,

$\boldsymbol{n}_{\mathrm{c}}-\mathbf{F A} \boldsymbol{n}=\left(\boldsymbol{n}_{\mathrm{c}}-\mathbf{F R} \boldsymbol{n}_{\mathrm{c}}\right)-\mathbf{F R} \boldsymbol{w}-\mathbf{F}(\mathbf{A}-\mathbf{R}) \boldsymbol{w}-\mathbf{F}(\mathbf{A}-\mathbf{R}) \boldsymbol{n}_{\mathrm{c}}$ 

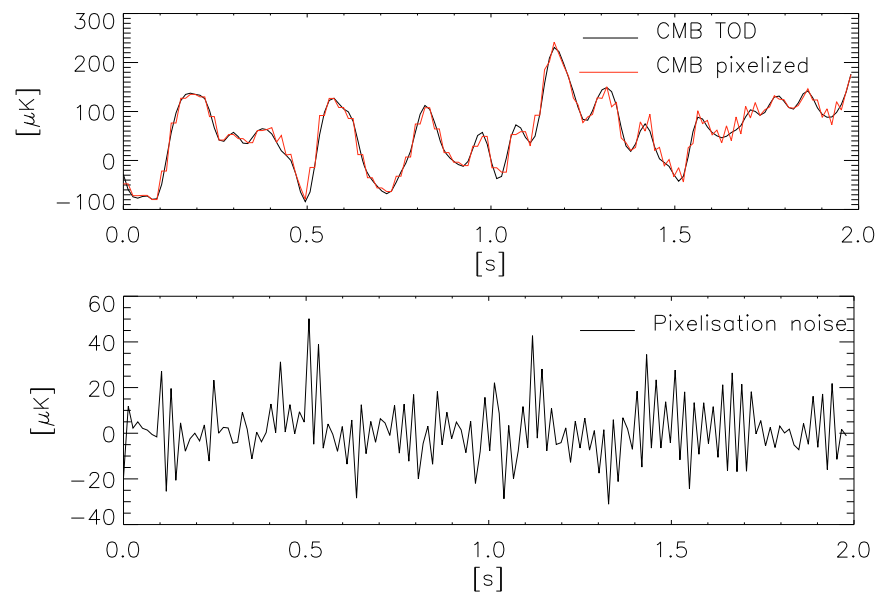

Fig. 20. Pixelization noise. In the top panel we show a piece of the original signal stream $\boldsymbol{s}$ (black) and the pixelized signal stream PBs (red). Their difference $\mathbf{Z} \boldsymbol{s}=\boldsymbol{s}-\mathbf{P B} \boldsymbol{s}$ is the pixelization noise (bottom panel).

The variances of the four components are $\sigma_{u}^{2}, \sigma_{w r}^{2}, \sigma_{w e}^{2}$, and $\sigma_{u b}^{2}$. The first three components are uncorrelated, so that their total variance is $\sigma_{u}^{2}+\sigma_{w r}^{2}+\sigma_{w e}^{2}$. Since $\sigma_{w e}^{2}$ and $\sigma_{u b}^{2}$ are much smaller than $\sigma_{u}^{2}+\sigma_{w r}^{2}$, we make the approximation where we drop the last two components.

Using Eqs. (49) and (53) we find that $\sigma_{w r}^{2}+\sigma_{u}^{2}$ is minimized for

$f_{x}=\frac{1}{2 t_{\text {base }}}=\left(1+\frac{\alpha-1}{5-\alpha}\right)^{1 / \alpha} f_{\mathrm{k}}$

for which

$\frac{\sigma_{w r}^{2}+\sigma_{u}^{2}}{\sigma^{2}} \approx\left(\frac{4}{5-\alpha}\right)^{1 / \alpha} \frac{\alpha}{\alpha-1}\left(\frac{f_{\mathrm{k}}}{f_{\mathrm{c}}}\right)-\frac{1}{\alpha-1}\left(\frac{f_{\mathrm{k}}}{f_{\mathrm{c}}}\right)^{\alpha}$.

For $\alpha=1$ this becomes

$f_{x}=\frac{1}{2 t_{\text {base }}}=f_{\mathrm{k}}$

and

$\frac{\sigma_{w r}^{2}+\sigma_{u}^{2}}{\sigma^{2}} \approx\left(\frac{5}{4}+\ln \frac{f_{\mathrm{c}}}{f_{\mathrm{k}}}\right) \frac{f_{\mathrm{k}}}{f_{\mathrm{c}}}$

For $f_{\mathrm{k}}=50 \mathrm{mHz}$ and $\alpha=1.7$ this gives $t_{\text {base }}=8.93 \mathrm{~s}$, for which $\sqrt{\sigma_{w r}^{2}+\sigma_{u}^{2}}=0.05936 \sigma=160.3 \mu \mathrm{K}$. This result does not take into account baseline errors and signal baselines (see Sect. 5.8).

However, because of the different correlation properties of the different residuals, they have a different impact in mapmaking, and it is not enough to consider the time-domain variance. So we need to return to this issue later, after we have studied the residuals in the map domain and their angular power spectra.

\subsection{Pixelization noise and signal baselines}

Pixelization noise, $\mathbf{Z} \boldsymbol{s} \equiv \boldsymbol{s}-\mathbf{P B} \boldsymbol{s}$, arises from signal gradients through a combination of pixelization, scanning strategy, and sampling frequency. These lead to correlations between close-by samples, and also correlations between samples from the same locations on the sky which are not close to each other in time domain.

In Fig. 20 we show a short piece of the pixelization noise. Its autocorrelation function is shown in Fig. 21. We see that

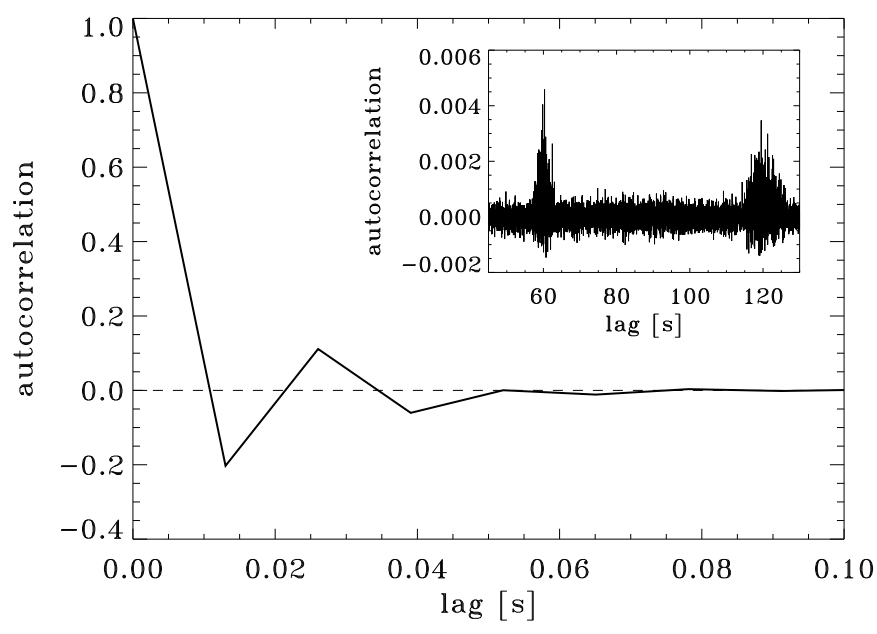

Fig. 21. Autocorrelation function of the pixelization noise. Note the alternating correlation and anticorrelation for small lags, and the correlations at $1 \mathrm{~min}$ lag, when the scanning returns to the same location on the sky. Due to spin rate variations the correlations are spread around the $1 \mathrm{~min}$ value, and more around $2 \mathrm{~min}$.

neighboring samples (lag $=1=(1 / 76.8)$ s) are anticorrelated, whereas we have a positive correlation for lag $=2$. The power spectrum is close to that of white noise, except that there are some features near the Nyquist frequency due to these correlations.

Comparing the pixel size $\theta_{p}=6.87^{\prime}$ to the sample separation $\theta_{\mathrm{s}}=4.68^{\prime}$ and remembering that the scanning direction is mostly close to the direction of the pixel diagonal (both are often close to the ecliptic meridians), so that the pixel geometry tends to repeat at $\sim \sqrt{2} \theta_{p}=9.71^{\prime}$ intervals, close to $2 \theta_{\mathrm{s}}=9.38^{\prime}$, we see that a pair of samples with lag 2 tends to land in about the same relative location within their respective pixels. With this small separation there is a positive correlation in the CMB signal gradient (smoothed with the beam). These combine to give a positive correlation between lag 2 samples. On the other hand, neighboring samples often land at opposite sides of the same pixel, or of neighboring pixels, leading to a negative correlation in their pixelization noise. These correlations would be different for different ratios of sample separation to pixel size.

Since the pixelization noise arises from the signal, we analyze it in the combinations $\boldsymbol{s}_{+} \equiv\left(\boldsymbol{s}_{a}+\boldsymbol{s}_{b}\right) / 2$ ("temperature") and $s_{-} \equiv\left(s_{a}-s_{b}\right) / 2$ ("polarization"), so that

$s_{+}=I \quad$ and $\quad s_{-}=Q \cos 2 \psi_{a}+U \sin 2 \psi_{a}$.

Each of the $s_{+}$and $s_{-}$time streams contains $n_{t} / 2$ samples. For the ideal detectors considered here, $\boldsymbol{s}_{+}$arises from gradients in the intensity of the signal, and $s_{-}$from gradients in the polarization of the signal. We assume the signal is statistically isotropic, and that $C_{\ell}^{E B}=0$.

We find that the expectation value for the variance of the pixelization noise is

$$
\begin{aligned}
\sigma_{p}^{2}(\text { temp }) \approx & (\Gamma-\Lambda) \sum_{\ell} \frac{(2 \ell+1) \ell(\ell+1)}{8 \pi} B_{\ell}^{2} C_{\ell}^{T T} \\
\sigma_{p}^{2}(\mathrm{pol}) \approx & (\Gamma-\Lambda) \sum_{\ell} \frac{(2 \ell+1)[(\ell-2)(\ell+3)+2]}{16 \pi} \\
& \times B_{\ell}^{2}\left(C_{\ell}^{E E}+C_{\ell}^{B B}\right)
\end{aligned}
$$




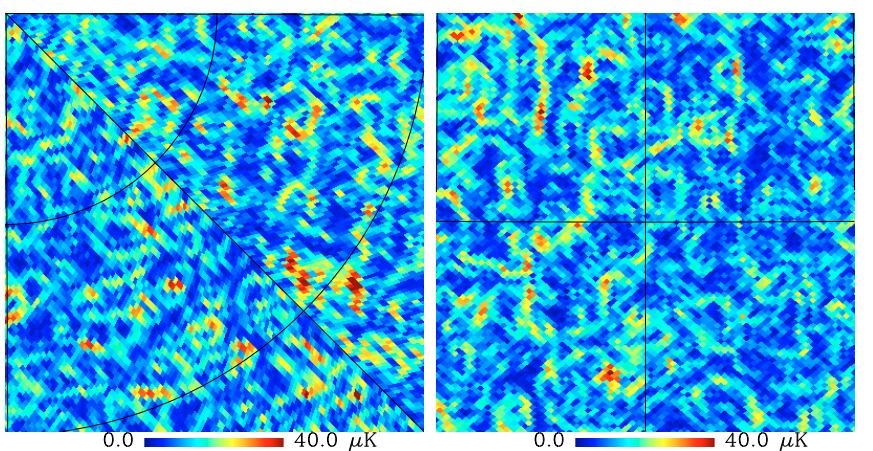

Fig. 22. Pixelization noise $\mathrm{rms}$ in map pixels for the two $10^{\circ} \times$ $10^{\circ}$ regions.

where

$\Gamma=\frac{1}{n_{t}} \sum_{p} \sum_{t \in p}\left|\boldsymbol{r}_{t}\right|^{2}, \quad \Lambda=\frac{1}{n_{t}} \sum_{p} \frac{1}{n_{\mathrm{hit}, p}} \sum_{t t^{\prime} \in p} \boldsymbol{r}_{t} \cdot \boldsymbol{r}_{t^{\prime}}$,

and $\boldsymbol{r}_{t} \equiv \boldsymbol{n}_{t}-\boldsymbol{u}_{p}$. Here $\boldsymbol{n}_{t}$ is the unit vector giving the pointing $\left(\theta_{t}, \phi_{t}\right)$ of sample $t$, and $\boldsymbol{u}_{p}$ is the unit vector pointing to the center of the pixel $p$ hit by sample $t$. The approximation in Eq. (63) is the small-pixel approximation $\left|\boldsymbol{r}_{t}\right| \ll 1$. For $\sigma_{p}$ (pol) we have also assumed an optimal distribution of polarization directions $\psi_{t}$ within each pixel.

This result, Eq. (63), can be compared to the variance of the signal itself

$$
\begin{aligned}
\sigma_{s}^{2}(\text { temp }) & \equiv\langle I(\boldsymbol{n}) I(\boldsymbol{n})\rangle=\sum_{\ell} \frac{2 \ell+1}{4 \pi} C_{\ell}^{T T} \\
\sigma_{s}^{2}(\mathrm{pol}) & \equiv\langle Q(\boldsymbol{n}) Q(\boldsymbol{n})\rangle=\langle U(\boldsymbol{n}) U(\boldsymbol{n})\rangle \\
& =\sum_{\ell} \frac{2 \ell+1}{8 \pi}\left(C_{\ell}^{E E}+C_{\ell}^{B B}\right) .
\end{aligned}
$$

Assuming the pixels are perfect squares, in the limit of a large number of hits $n_{\text {hit, } p}$ uniformly distributed over the pixel we get

$\Gamma \approx \frac{\Omega_{p}}{6} \quad$ and $\quad \Lambda \ll \Gamma$.

Since HEALPix pixels are not square, but somewhat elongated, we expect the actual $\Gamma-\Lambda$ to be somewhat larger. In principle it can be calculated from the pointing data for a chosen pixelization.

For our input $C_{\ell}$, setting $\Gamma-\Lambda=\Omega_{p} / 6$, Eqs. (63) and (65) give $\sigma_{s}($ temp $)=100.792 \mu \mathrm{K}, \sigma_{p}($ temp $)=13.332 \mu \mathrm{K}, \sigma_{s}($ pol $)=$ $2.530 \mu \mathrm{K}$, and $\sigma_{p}(\mathrm{pol})=0.781 \mu \mathrm{K}$.

The actual pixelization noise level in the simulation was $\sigma_{p}($ temp $)=13.516 \mu \mathrm{K}$ and $\sigma_{p}(\mathrm{pol})=0.776 \mu \mathrm{K}$.

By definition, a binned map of pixelization noise vanishes, $\mathbf{B Z} \boldsymbol{s}=\mathbf{B} \boldsymbol{s}-\mathbf{B P B} \boldsymbol{s}=0$. Instead we can make a map of the rms of the pixelization noise at each pixel, by squaring each element of $\mathbf{Z s}$, binning the resulting time stream into a map, and taking the square root of each I pixel. See Fig. 22. Comparing to Fig. 3 we see that pixels of large pixelization noise tend to "outline" hot and cold spots of the signal.

Signal baselines arise from the pixelization noise in the same manner as white noise baselines arise from white noise, and we can divide them into the reference baselines of pixelization noise, and signal baseline errors, $\mathbf{A} \boldsymbol{s}=\mathbf{R Z} \boldsymbol{s}+(\mathbf{A}-\mathbf{R Z}) \boldsymbol{s}$.
Table 7. Stdev of contributions to signal baselines.

\begin{tabular}{cccccc}
\hline \hline$t_{\text {base }}$ & $\begin{array}{c}\text { Eq. (67) } \\
(\mu \mathrm{K})\end{array}$ & $\begin{array}{c}\text { RZs } \\
(\mu \mathrm{K})\end{array}$ & $\begin{array}{c}(\mathbf{A}-\mathbf{R Z}) \boldsymbol{s} \\
(\mu \mathrm{K})\end{array}$ & $\begin{array}{c}\mathbf{A s} \\
(\mu \mathrm{K})\end{array}$ & $\begin{array}{c}\mathbf{A} \boldsymbol{s}(\mathrm{G}) \\
(\mu \mathrm{K})\end{array}$ \\
\hline \multicolumn{5}{c}{ temperature } & stream $(a+b) / 2$ \\
$2.5 \mathrm{~s}$ & 0.962 & 0.972 & 0.672 & 1.297 & $\ldots$ \\
$5 \mathrm{~s}$ & 0.680 & 0.686 & 0.300 & 0.831 & $\ldots$ \\
$15 \mathrm{~s}$ & 0.393 & 0.396 & 0.121 & 0.461 & $\ldots$ \\
$1 \mathrm{~min}$ & 0.196 & 0.200 & 0.075 & 0.248 & 0.243 \\
$1 \mathrm{~h}$ & 0.136 & 0.075 & 0.199 & 0.196 \\
\hline \multicolumn{5}{c}{ polarization stream $(a-b) / 2$} \\
$2.5 \mathrm{~s}$ & 0.0564 & 0.0560 & 0.0277 & 0.0701 & $\ldots$ \\
$5 \mathrm{~s}$ & 0.0399 & 0.0396 & 0.0145 & 0.0476 & $\ldots$ \\
$15 \mathrm{~s}$ & 0.0230 & 0.0229 & 0.0068 & 0.0269 & $\ldots$ \\
$1 \mathrm{~min}$ & 0.0115 & 0.0115 & 0.0043 & 0.0144 & 0.0147 \\
$1 \mathrm{~h}$ & & 0.0083 & 0.0043 & 0.0120 & 0.0124 \\
\hline
\end{tabular}

Approximating the pixelization noise as white, we get an estimate for the standard deviation (stdev) of $\mathbf{R Z s}$

$$
\begin{aligned}
& \sigma_{p r}(\text { temp }) \approx \frac{\sigma_{p}(\text { temp })}{\sqrt{n_{\text {base }}}} \approx 1.521 \mu \mathrm{K} t_{\text {base }}(\mathrm{s})^{-1 / 2}, \\
& \sigma_{p r}(\text { pol }) \approx \frac{\sigma_{p}(\text { pol })}{\sqrt{n_{\text {base }}}} \approx 0.0891 \mu \mathrm{K} t_{\text {base }}(\mathrm{s})^{-1 / 2} .
\end{aligned}
$$

In Table 7 we show the stdev of the signal baselines $\mathbf{A} \boldsymbol{s}$ and their two contributions RZs (reference baselines of pixelization noise) and (A - RZ)s (signal baseline error), and compare them to the estimate (67) for RZs. We see the estimate is quite good for RZs. The estimate is slightly off mainly because the actual pixelization noise was slightly larger for temperature and slightly smaller for polarization than the analytical estimate (63). The column marked "(G)" was obtained using galactic coordinates for the HEALPix maps.

In Fig. 23 we show the autocorrelation functions of the reference baselines of pixelization noise and the signal baseline error. Unlike for white noise, now also the reference baselines are correlated. This correlation extends over several repointing periods. These correlations enhance the relevance of the signal baselines in the map domain.

\section{Map domain}

Destriping is a linear process. The output map can therefore be viewed as a sum of component maps, each component map being a result of destriping one individual TOD component: signal, $1 / f$ noise and white noise:

$$
\boldsymbol{m}_{\mathrm{out}}=\mathbf{B}(\boldsymbol{s}-\mathbf{F A} s)+\mathbf{B}\left(\boldsymbol{n}_{\mathrm{c}}-\mathbf{F A} \boldsymbol{n}_{\mathrm{c}}\right)+\mathbf{B}(\boldsymbol{w}-\mathbf{F A} w) .
$$

As the three TOD components are statistically independent, so are the corresponding component maps. The expectation value of the map rms is thus obtained as the root sum square (rss) of the expectation values of the rms of the component maps.

In this paper we have considered a single 1-year realization of each TOD component only. There are likely to be random correlations between the component maps. However, the output map and residual map rms we get when we produce maps from the full TOD, agree to better than $0.5 \%$ with the rss of the rms of the corresponding maps produced from component TODs.

\subsection{Linearity}

In practice the linearity of destriping is affected by the numerical accuracy of the baseline calculation, which depends on the 

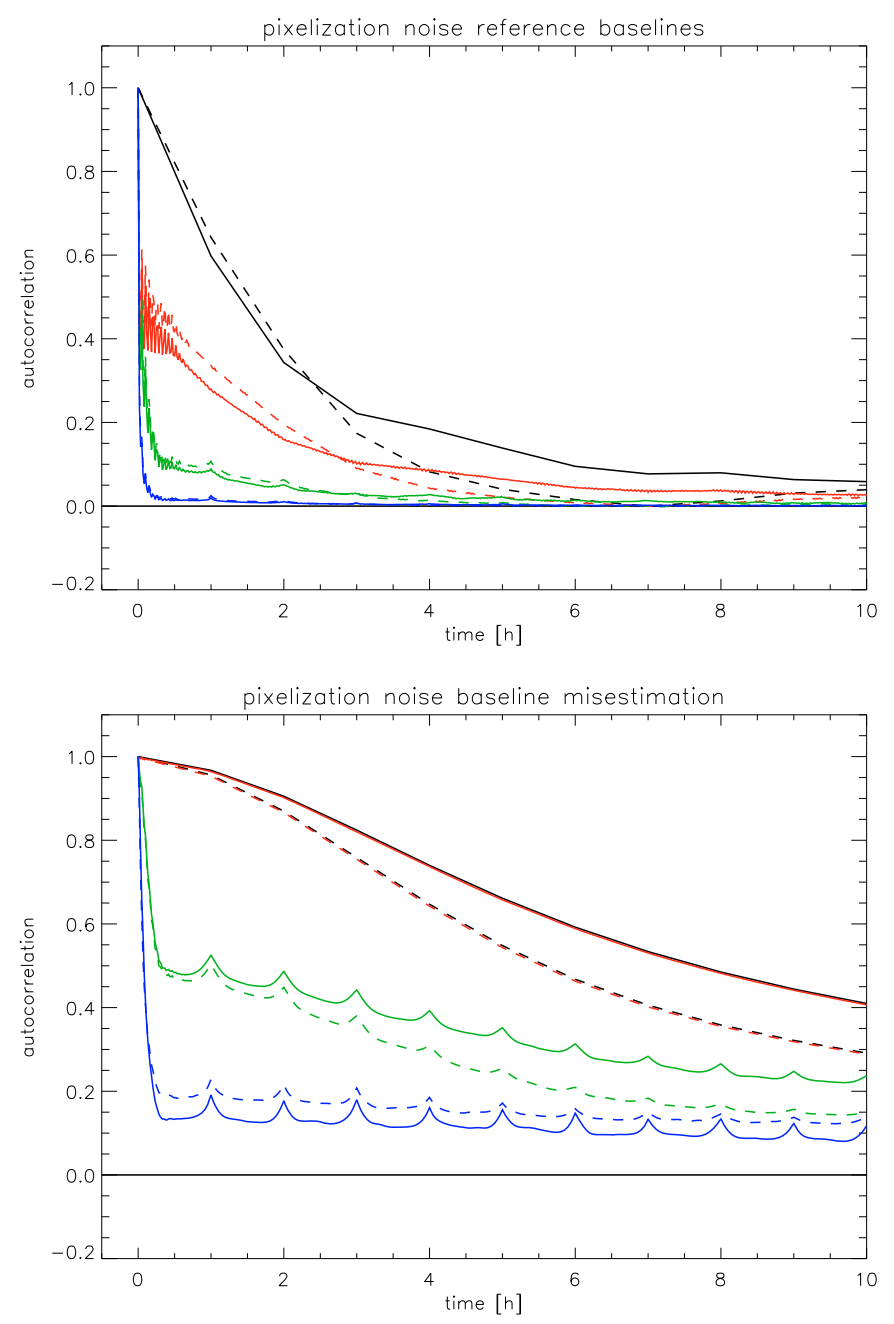

Fig. 23. Correlations in the signal baselines. The top panel shows the autocorrelation function of the reference baselines RZs of pixelization noise, and the bottom panel that of the signal baseline error $(\mathbf{A}-\mathbf{R Z}) \boldsymbol{s}$. The colors and line styles are as in Fig. 11.

convergence criterion for the conjugate gradient iteration. We checked the linearity by comparing the sum of the component maps to a map obtained directly from the sum of the component TODs. The difference is well below the $\mathrm{nK}$ level, except for baselines shorter than 10 s. See Fig. 24.

\subsection{Splitting the residual map into components}

The three component maps can each be further divided into a binned map and a baseline map.

The correlation properties of these components are different in the map domain from the TOD domain.

While the white noise baselines FA $\boldsymbol{w}$ are correlated with the white noise $\boldsymbol{w}$ in the TOD, the correlation vanishes in the map: writing

$$
\begin{aligned}
& \mathbf{B} \boldsymbol{w}=\mathbf{M}^{-1} \mathbf{P}^{\mathrm{T}} \mathbf{C}_{w}^{-1} \boldsymbol{w} \\
& \mathbf{B F A} \boldsymbol{w}=\mathbf{M}^{-1} \mathbf{P}^{\mathrm{T}} \mathbf{C}_{w}^{-1} \mathbf{F} \mathbf{D}^{-1} \mathbf{F}^{\mathrm{T}} \mathbf{C}_{w}^{-1}\left(\mathbf{I}-\mathbf{P M}^{-1} \mathbf{P}^{\mathrm{T}} \mathbf{C}_{w}^{-1}\right) \boldsymbol{w}
\end{aligned}
$$

we get that

$$
\begin{aligned}
\left\langle(\mathbf{B} \boldsymbol{w})(\mathbf{B F A} \boldsymbol{w})^{\mathrm{T}}\right\rangle= & \mathbf{M}^{-1} \mathbf{P}^{\mathrm{T}} \mathbf{C}_{w}^{-1}\left\langle\boldsymbol{w} \boldsymbol{w}^{\mathrm{T}}\right\rangle\left(\mathbf{I}-\mathbf{C}_{w}^{-1} \mathbf{P} \mathbf{M}^{-1} \mathbf{P}^{\mathrm{T}}\right) \\
& \mathbf{C}_{w}^{-1} \mathbf{F D}^{-1} \mathbf{F}^{\mathrm{T}} \mathbf{C}_{w}^{-1} \mathbf{P} \mathbf{M}^{-1} .
\end{aligned}
$$

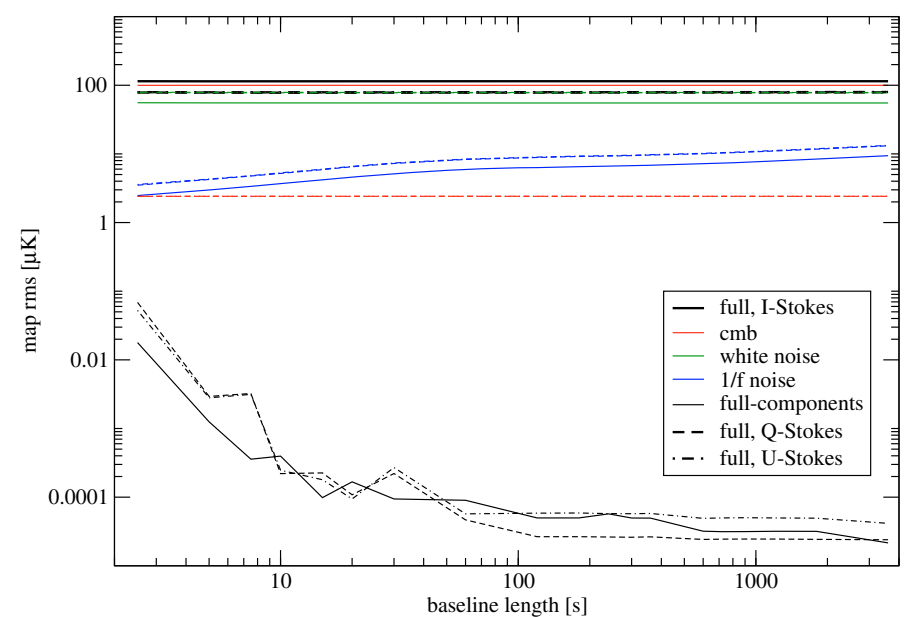

Fig. 24. Linearity of destriping. The three thin black lines at the bottom shows the rms difference between the "full" map, obtained from the sum of the component TODs, and the sum of the component maps. This is compared to the signal and noise levels in the maps; different colors showing the different components and the thick black line the full map; different line styles correspond to the three Stokes parameters.

By substituting $\left\langle\boldsymbol{w} \boldsymbol{w}^{\mathrm{T}}\right\rangle=\mathbf{C}_{w}$ one readily sees that the correlation vanishes,

$\left\langle(\mathbf{B} w)(\mathbf{B F A} w)^{\mathrm{T}}\right\rangle=0$.

Because the binned white noise map and the white noise baseline map are independent, the (expectation value of) the rms of the residual white noise map $\mathbf{B} \boldsymbol{w}-\mathbf{B F A} \boldsymbol{w}$ is obtained as the rss of the rms of the two maps.

This means, that while subtracting the white noise baselines removes power from the TOD, it adds power to the map. If the noise is pure white, naive binning produces a better map than destriping. On the other hand, baselines of the $1 / f$ noise are correlated with the $1 / f$ noise itself both in the TOD and in the map.

We can use the rms value taken over all the residual map pixels as a figure-of-merit for the map-making method. We calculate it separately for the three Stokes parameters, I (temperature), $Q$, and $U$. Note that we subtract the I monopole from the residual map before calculating the rms, since it is irrelevant for CMB anisotropy and polarization studies, and destriping leaves a spurious $I$ monopole in the map. See Figs. 25-27.

The difference between the output map and the binned signal map

$\boldsymbol{m}_{\text {out }}-\mathbf{B} \boldsymbol{s}=\mathbf{B} w-\mathbf{B F A} w+\mathbf{B}\left(\boldsymbol{n}_{\mathrm{c}}-\mathbf{F A} \boldsymbol{n}_{\mathrm{c}}\right)-\mathbf{B F A} \boldsymbol{s}$

is the residual map including binned white noise. We see that it can be divided into four uncorrelated contributions: the binned white noise map, the white noise baseline map, the residual $1 / f$ noise map, and the signal baseline map.

The $\boldsymbol{m}_{\text {out }}-\mathbf{B} \boldsymbol{s}$ I map is shown in Fig. 28. This map is dominated by the binned white noise map, which is independent of the baseline length. Therefore the visual appearance of the residual map is the same for all baseline lengths, as it looks the same as the white noise map. Also the residual $Q$ and $U$ maps look the same, just with $\sim \sqrt{2}$ larger amplitude.

The binned white noise map is independent of the baseline length, and it is an unavoidable component of the residual map, uncorrelated with the other components. Therefore we focus on the rest of the residual map, without the binned white noise component, i.e.,

$m_{\text {out }}-\mathbf{B} s-\mathbf{B} w=-\mathbf{B F A} w+\mathbf{B}\left(\boldsymbol{n}_{\mathrm{c}}-\mathbf{F A} \boldsymbol{n}_{\mathrm{c}}\right)-$ BFAs. 

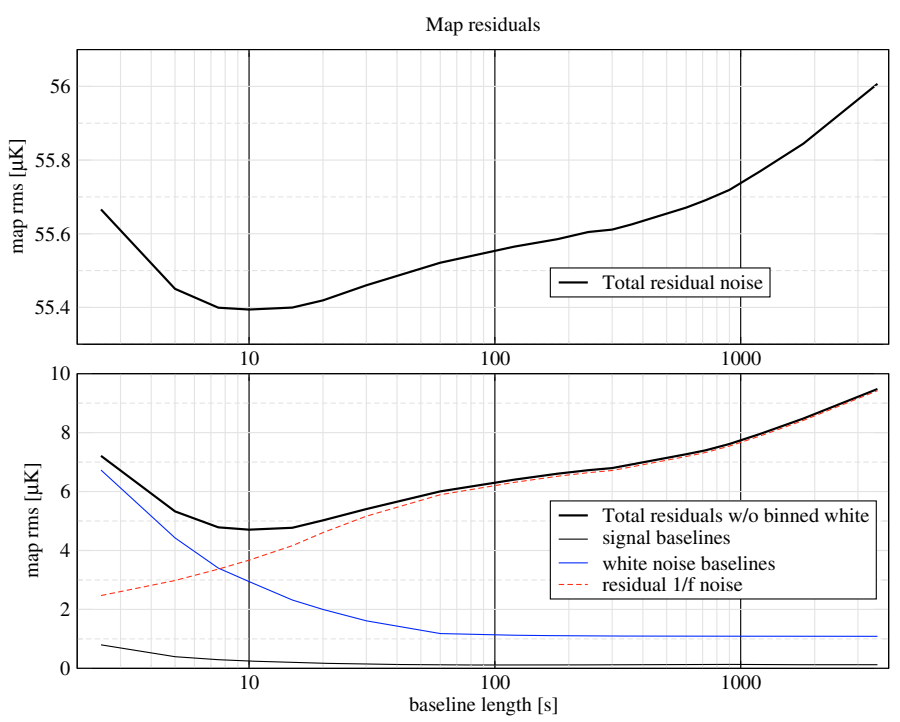

Fig. 25. Residual temperature map rms as a function of baseline length. The top panel is for $\boldsymbol{m}_{\text {out }}-\mathbf{B} \boldsymbol{s}$, which is dominated by the binned white noise map, whose rms is $55.19 \mu \mathrm{K}$ independent of $t_{\text {base }}$. The bottom panel shows the other three components, and their root sum square, which is the rms of the residual map $\boldsymbol{m}_{\text {out }}-\mathbf{B} \boldsymbol{s}-\mathbf{B} \boldsymbol{w}$.

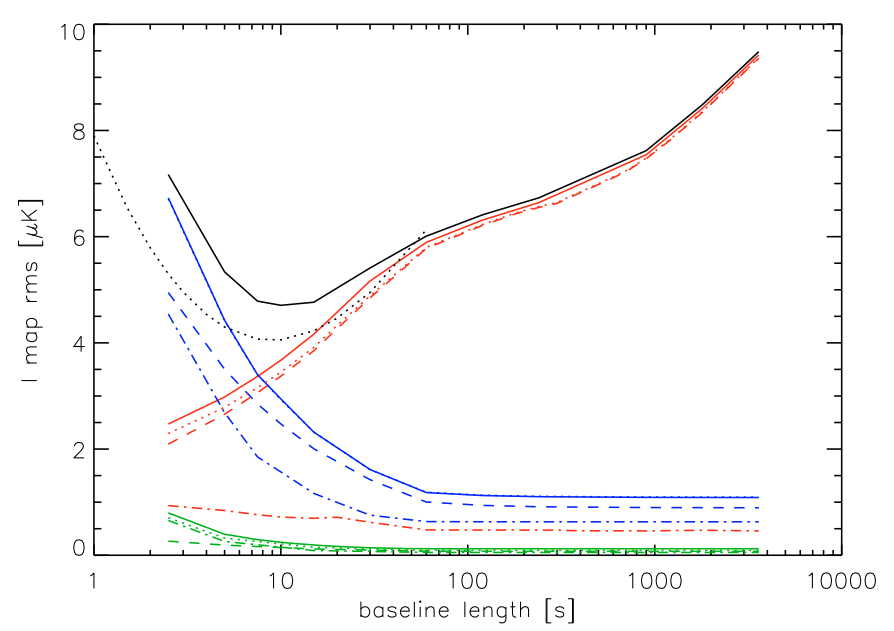

Fig. 26. Same as the bottom panel of Fig. 25, but now we have further separated the residual $1 / f$ noise into unmodeled $1 / f$ noise (dashed) and $1 / f$ baseline error (dot-dashed); the white noise baselines into reference baselines (dashed) and baseline error (dot-dashed); and signal baselines (shown here in green) into reference baselines of pixelization noise (dashed) and baseline error (dot-dashed). The dotted lines show the rss of the dashed and dot-dashed lines. For white noise it falls on the solid line, showing that the two white noise residual map components are uncorrelated. For $1 / f$ noise and pixelization noise the two components are positively correlated. The black dotted line is the analytical approximation Eq. (86).

For the rest of this paper, the residual map without further qualification, refers to this map. See Figs. 29 and 30.

We divide this further into

$$
\begin{aligned}
\boldsymbol{m}_{\mathrm{out}}-\mathbf{B} \boldsymbol{s}-\mathbf{B} \boldsymbol{w}= & -\mathbf{B F R} \boldsymbol{w}-\mathbf{B F}(\mathbf{A}-\mathbf{R}) \boldsymbol{w} \\
& +\mathbf{B}\left(\boldsymbol{n}_{\mathrm{c}}-\mathbf{F} \boldsymbol{a}_{r}\right)-\mathbf{B F}\left(\mathbf{A} \boldsymbol{n}_{\mathrm{c}}-\boldsymbol{a}_{r}\right) \\
& -\mathbf{B F R Z} \boldsymbol{s}-\mathbf{B F}(\mathbf{A}-\mathbf{R Z}) \boldsymbol{s},
\end{aligned}
$$
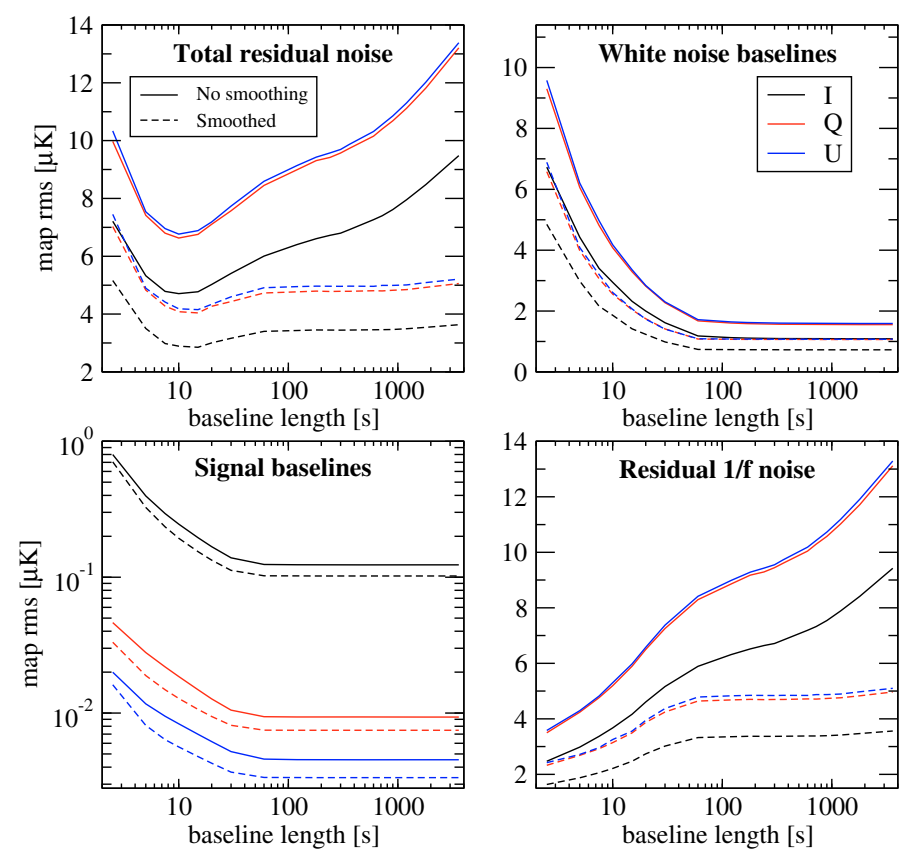

Fig. 27. The rms of the residual $I, Q$, and $U$ maps, and their different components. The overall residual power in a destriped polarization map is higher than in a temperature map. The CMB signal residual in turn is much weaker. Note how $Q$ is larger than $U$ in the signal baselines. Smoothing the residual maps with a Gaussian beam (dashed lines) removes excess power at sub-beam scales.

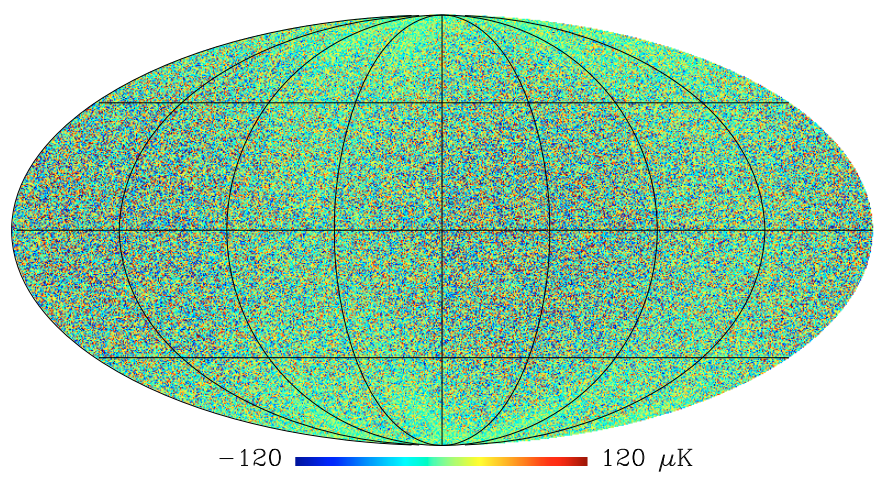

Fig. 28. Residual $I$ map including binned white noise, $\boldsymbol{m}_{\text {out }}-\mathbf{B} \boldsymbol{s}$, for $t_{\text {base }}=1 \mathrm{~min}$. It looks the same for other baseline lengths. Also the binned white noise map $\mathbf{B} \boldsymbol{w}$ looks the same, as it is the dominant component in the residual map. The $Q$ and $U$ maps look the same, but have a larger amplitude.

in analogy with Eq. (48). Of these six components, the unmodeled $1 / f \operatorname{map} \mathbf{B}\left(\boldsymbol{n}_{\mathrm{c}}-\mathbf{F} \boldsymbol{a}_{r}\right)$ is correlated with the $1 / f$ baseline error map $\mathbf{B F}\left(\mathbf{A} \boldsymbol{n}_{\mathrm{c}}-\boldsymbol{a}_{r}\right)$, and the pixelization noise reference baseline map BFRZs is correlated with the signal baseline error map $\mathbf{B F}(\mathbf{A}-\mathbf{R Z})$ s. Otherwise the components are uncorrelated with each other. The division is useful, since the different components have a different structure in the map domain. The unmodeled $1 / f$ map consists of different noise frequencies with half-wavelength mostly of the same order or shorter than the baseline (Fig. 15). The other 5 components consist of baselines laid on the map. For the white noise reference baseline map, these baselines are uncorrelated with each other, the other 4 components have different levels of correlation. In Fig. 31 we 

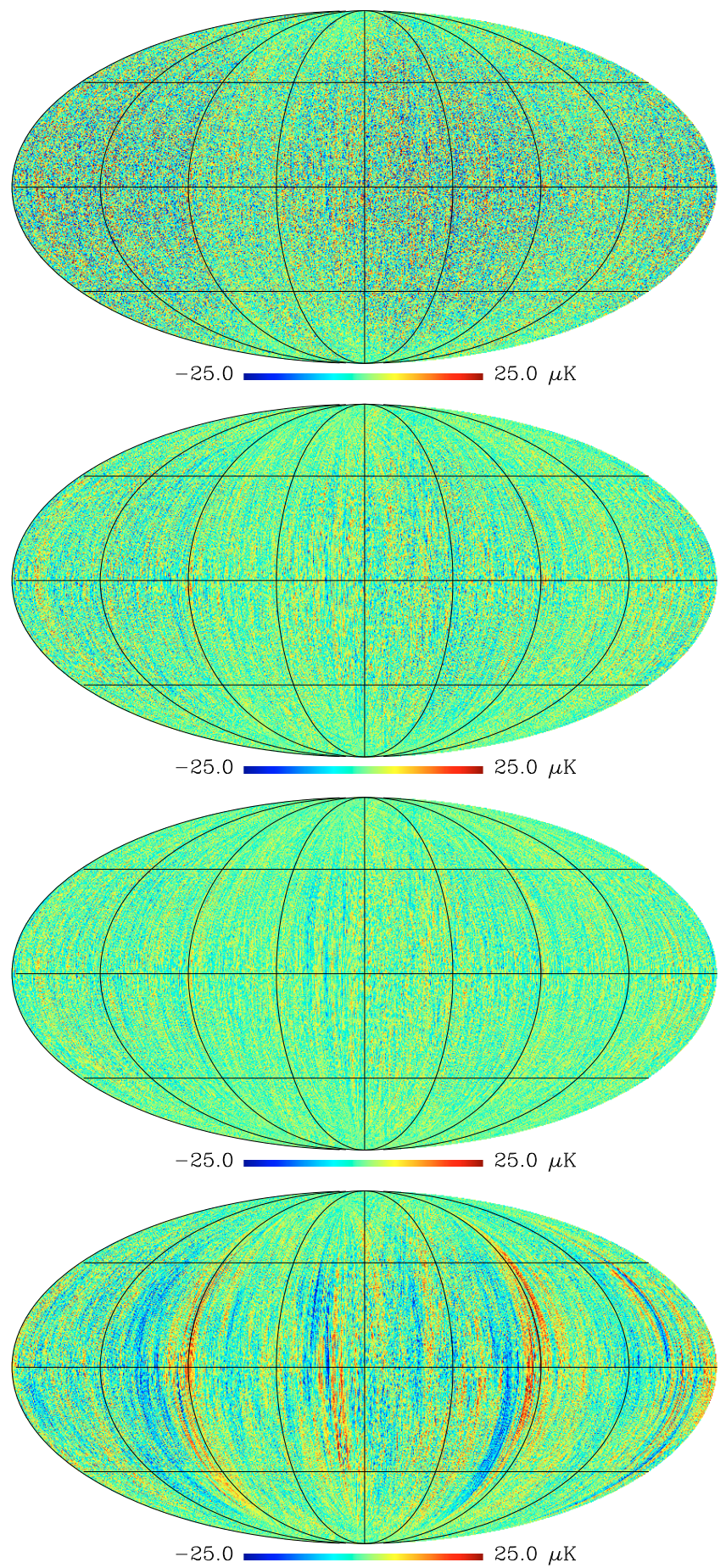

Fig. 29. Residual $I$ map (with binned white noise subtracted), $\boldsymbol{m}_{\text {out }}-\mathbf{B} \boldsymbol{s}-\mathbf{B} \boldsymbol{w}$, for different baseline lengths (from top down:) $1 \mathrm{~h}$, $1 \mathrm{~min}, 15 \mathrm{~s}$, and $2.5 \mathrm{~s}$. The $Q$ and $U$ maps look the same with $\sim \sqrt{2}$ larger amplitude.

show each of the six components for a $10^{\circ} \times 10^{\circ}$ region of the I map.

In Fig. 26, we show separately the rms of these components.

\subsection{Analytical estimates}

\subsubsection{White noise baselines}

We show in Table 8 the rms of the white noise reference baseline maps. Since the white noise reference baselines are uncorrelated
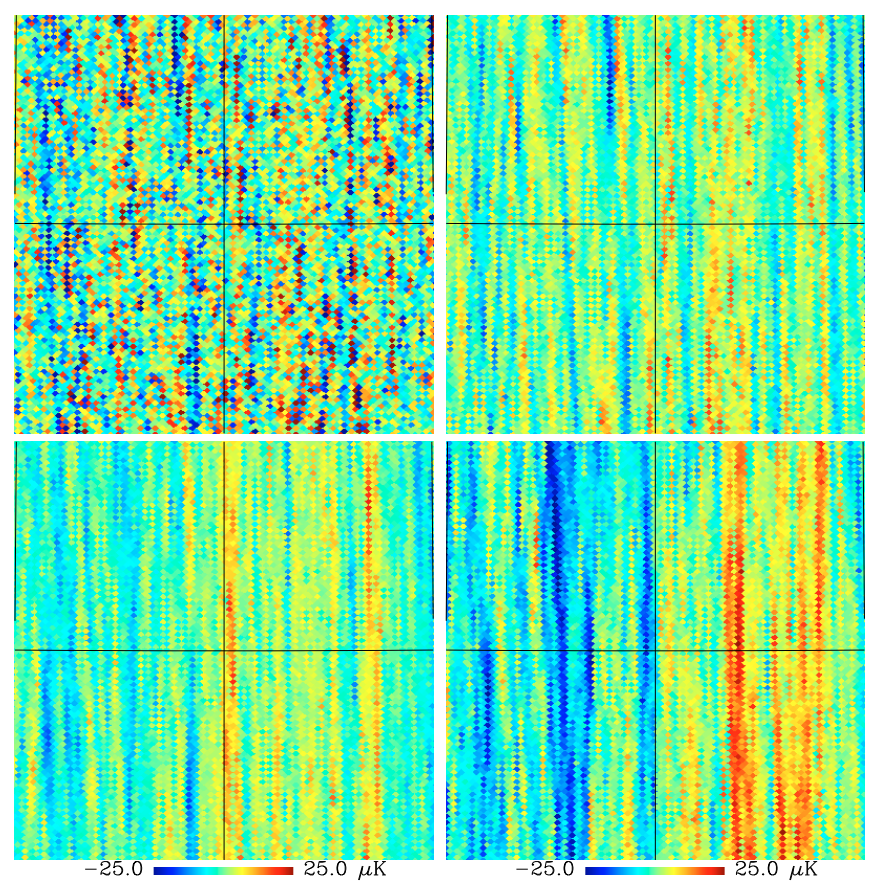

Fig. 30. Same as Fig. 29 but for the $10^{\circ} \times 10^{\circ}$ region near the ecliptic. Top left: $t_{\text {base }}=1$ h. Top right: $t_{\text {base }}=1 \mathrm{~min}$. Bottom left: $t_{\text {base }}=15 \mathrm{~s}$. Bottom right: $t_{\text {base }}=2.5 \mathrm{~s}$.

with each other, these map rms can be estimated analytically. If all hits to a pixel came from a different baseline, we could treat them as white noise, with the white noise reference baseline variance $\sigma_{w r}^{2}=\sigma^{2} / n_{\text {base }}$. Thus the variances of $I_{p}, Q_{p}$, and $U_{p}$ would be just $\left(M_{p}\right)^{-1}(1,1) / n_{\text {base }} \approx \sigma^{2} / n_{\text {base }} / n_{\text {hit }, p},\left(M_{p}\right)^{-1}(2,2) / n_{\text {base }} \approx$ $2 \sigma^{2} / n_{\text {base }} / n_{\text {hit }, p}$, and $\left(M_{p}\right)^{-1}(3,3) / n_{\text {base }} \approx 2 \sigma^{2} / n_{\text {base }} / n_{\text {hit }, p}$.

For baseline lengths less than or equal to the spin period, $t_{\text {base }} \leq 1 \mathrm{~min}$, this holds if the sample separation is larger than the pixels, $\theta_{\mathrm{s}} \gg \theta_{p}$. However, in our case $\theta_{\mathrm{s}}<\theta_{p}$, and two or three successive samples may hit the same pixel. These successive hits are then almost always from the same baseline, and, for the baseline components, fully correlated, i.e., equal.

Denote by $n f_{n}$ the fraction of hits to a pixel that belong to a sequence of exactly $n$ successive hits to the same pixel, i.e, there are $f_{n} n_{\text {hit, } p}$ such sequences, and $\sum_{n} n f_{n}=1$. The variance of $I_{p}$ is then

$\left\langle I_{p}^{2}\right\rangle=\left(\sum_{n}\left(n^{2} f_{n}\right)\right) \frac{\sigma_{w b}^{2}}{n_{\mathrm{hit}, p}}$,

where $\sum_{n}\left(n^{2} f_{n}\right) \geq 1$.

With Eq. (49) we have for the I map rms

$I_{\mathrm{rms}} \approx \sqrt{\left\langle\frac{\sum n^{2} f_{n}}{n_{\text {hit }}}\right\rangle} \frac{\sigma}{\sqrt{n_{\text {base }}}}$.

To get an estimate for $\left\langle\sum_{n}\left(n^{2} f_{n}\right)\right\rangle$ we assume a square pixel and a uniform hit probability distribution within the pixel area.

For scanning in the direction of the pixel diagonal, for $\theta_{\mathrm{s}}>$ $\sqrt{2} \theta_{p}$ there are no successive hits to the same pixel and $f_{1}=$ $\sum_{n}\left(n^{2} f_{n}\right)=1$. For $\theta_{p} / \sqrt{2}<\theta_{\mathrm{s}}<\sqrt{2} \theta_{p}$ there can be a maximum of two hits, with $\left\langle f_{2}\right\rangle=(1-r / \sqrt{2})^{2}$ and

$\sum_{n} n^{2} f_{n}=3-2 \sqrt{2} r+r^{2}$ 

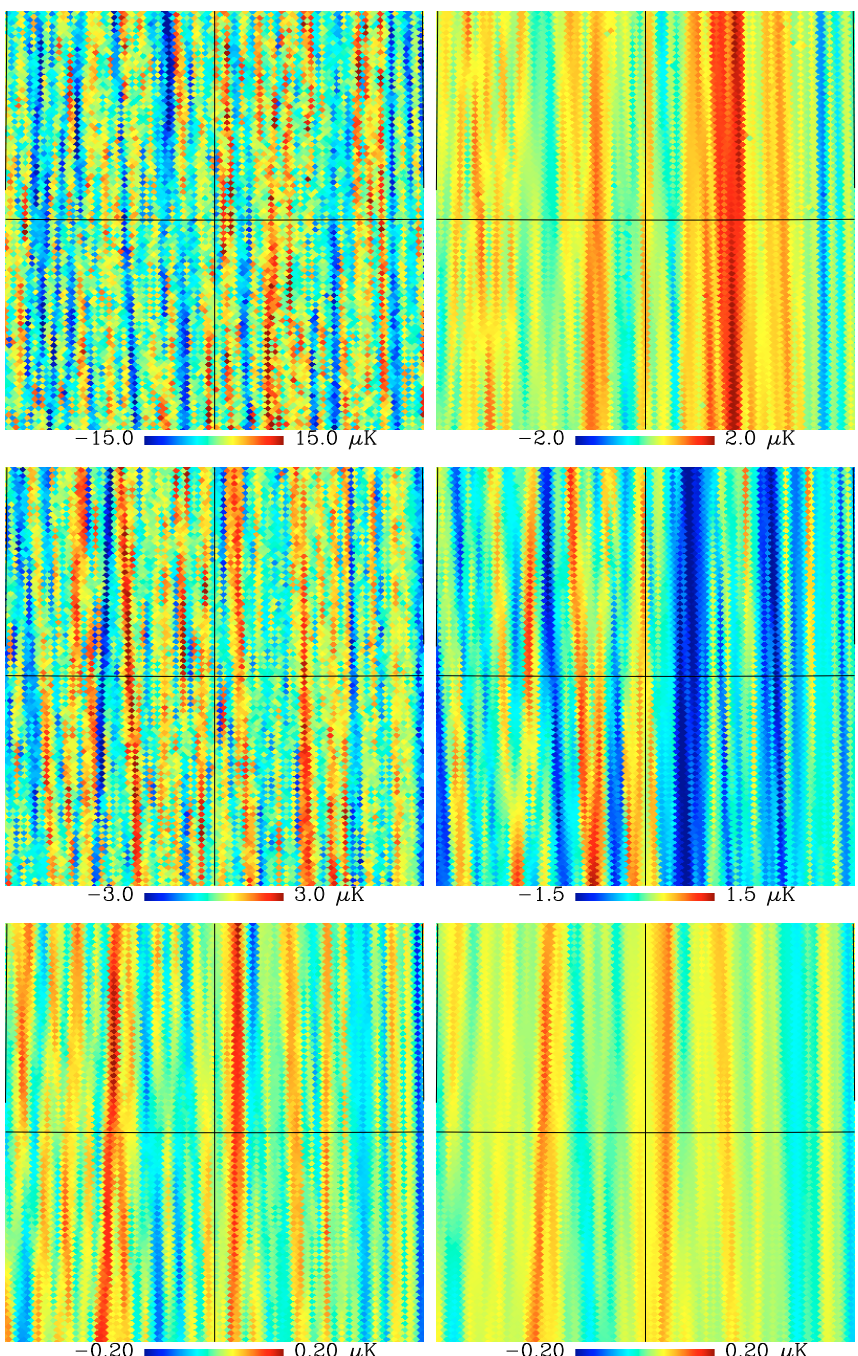

Fig. 31. The six components of the residual $I$ map shown for the $10^{\circ} \times 10^{\circ}$ region near the ecliptic $\left(t_{\text {base }}=1 \mathrm{~min}\right)$. Top left: unmodeled $1 / f$ noise $\mathbf{B}\left(\boldsymbol{n}_{\mathrm{c}}-\mathbf{F} \boldsymbol{a}_{r}\right)$. Top right: $1 / f$ baseline error $\mathbf{B F}\left(\mathbf{A} \boldsymbol{n}_{\mathrm{c}}-\boldsymbol{a}_{r}\right)$. Middle left: white noise reference baselines $-\mathbf{B F R} w$. Middle right: white noise baseline error $-\mathbf{B F}(\mathbf{A}-\mathbf{R}) \boldsymbol{w}$. Bottom left: pixelization noise reference baselines -BFRZs. Bottom right: signal baseline error $-\mathbf{B F}(\mathbf{A}-\mathbf{R Z}) \boldsymbol{s}$.

and with $\sqrt{2} \theta_{p} / 3<\theta_{\mathrm{s}}<\theta_{p} / \sqrt{2}$ a maximum of three hits, with $\left\langle f_{1}\right\rangle=r^{2},\left\langle f_{2}\right\rangle=3 \sqrt{2} r-1-7 r^{2} / 2$ and

$\sum_{n}\left(n^{2} f_{n}\right)=5-6 \sqrt{2} r+5 r^{2}$

where $r \equiv \theta_{\mathrm{s}} / \theta_{p}$.

For scanning in the direction of the pixel side, two hits, but no more, are possible for $\theta_{p} / 2<\theta_{\mathrm{s}}<\theta_{p}$ with $f_{2}=1-r$.

For our case, $\sqrt{2} / 3<1 / 2<r=0.682<1 / \sqrt{2}$, which gives

$$
\left\langle\sum_{n}\left(n^{2} f_{n}\right)\right\rangle=1.54=(1.24)^{2}
$$

for scanning in the diagonal direction, and $\left\langle\sum_{n}\left(n^{2} f_{n}\right)\right\rangle=1.64=$ $(1.28)^{2}$ for the pixel side direction.

However, HEALPix pixels are not square, but can be significantly elongated. In principle, the mean value $\left\langle\frac{\sum n^{2} f_{n}}{n_{\text {hit }}}\right\rangle$ could be calculated from the pointing data and the chosen pixelization. Here we just take it by comparing the actual $I_{\mathrm{rms}}$ from the maps
Table 8. rms of the white noise reference baseline maps.

\begin{tabular}{ccccc}
\hline \hline$t_{\text {base }}$ & $\begin{array}{c}I_{\text {rms }} \\
(\mu \mathrm{K})\end{array}$ & $\left\langle\left\langle\sum_{n} n^{2} f_{n}\right\rangle\right\rangle$ & $\begin{array}{c}Q_{\mathrm{rms}} \\
(\mu \mathrm{K})\end{array}$ & $\begin{array}{c}U_{\mathrm{rms}} \\
(\mu \mathrm{K})\end{array}$ \\
\hline $2.5 \mathrm{~s}$ & 4.947 & 1.542 & 6.974 & 7.072 \\
$15 \mathrm{~s}$ & 2.006 & 1.521 & 2.846 & 2.914 \\
$1 \mathrm{~min}$ & 1.003 & 1.522 & 1.435 & 1.466 \\
$1 \mathrm{~h}$ & 0.895 & & 1.289 & 1.320 \\
\hline
\end{tabular}

Table 9. rms of the white noise baseline error maps.

\begin{tabular}{ccccc}
\hline \hline$t_{\text {base }}$ & $\begin{array}{c}I_{\mathrm{rms}} \\
(\mu \mathrm{K})\end{array}$ & $c$ & $\begin{array}{c}Q_{\mathrm{rms}} \\
(\mu \mathrm{K})\end{array}$ & $\begin{array}{c}U_{\mathrm{rms}} \\
(\mu \mathrm{K})\end{array}$ \\
\hline $2.5 \mathrm{~s}$ & 4.543 & 0.168 & 6.223 & 6.433 \\
$15 \mathrm{~s}$ & 1.165 & 0.092 & 1.664 & 1.666 \\
$1 \mathrm{~min}$ & 0.634 & 0.102 & 0.881 & 0.904 \\
$1 \mathrm{~h}$ & 0.630 & & 0.875 & 0.898 \\
\hline
\end{tabular}

to Eq. (77). (See Table 8, where the third column is an estimate of $\left\langle\frac{\sum n^{2} f_{n}}{n_{\text {hit }}}\right\rangle /\left\langle n_{\text {hit }}^{-1}\right\rangle$ obtained this way.) This gives (from $t_{\text {base }}=15 \mathrm{~s}$ and $1 \mathrm{~min})\left\langle\sum n^{2} f_{n} / n_{\text {hit }}\right\rangle \approx 0.000636 \approx 1.52\left\langle n_{\text {hit }}^{-1}\right\rangle \approx(0.025)^{2}$. We denote

$\left\langle\left\langle\sum n^{2} f_{n}\right\rangle\right\rangle \equiv\left\langle\frac{\sum n^{2} f_{n}}{n_{\text {hit }}}\right\rangle\left\langle\left\langle n_{\text {hit }}^{-1}\right\rangle \approx 1.52\right.$.

Equation (80) is quite close to this.

For the other Stokes parameters, we expect

$Q_{\mathrm{rms}} \approx U_{\mathrm{rms}} \approx \sqrt{2} I_{\mathrm{rms}}$

where the approximation corresponds to assuming an ideal distribution of polarization directions. We expect this approximation to be good for the full year data, since most pixels have rcond values close to 0.5 .

For baselines longer than the spin period, contributions to a pixel from successive scan circles tend to come from the same baseline, so the reduction in the white noise baseline variance is canceled by the reduction in the number of contributing baselines. Thus the map rms from white noise baselines is almost flat between $t_{\text {base }}=1 \mathrm{~min}$ and $t_{\text {base }}=1 \mathrm{~h}$.

Table 9 shows the rms of the white noise baseline error maps. Although in the time domain the white noise baseline error is much smaller than white noise reference baselines, their correlations make them important in the map domain. Assuming the correlation between $(a+b) / 2$ baselines contributing to the same pixel were $c$, the expected variance of the white noise baseline error map would be

$\left\langle I_{s-r}^{2}\right\rangle=\left\langle I_{\text {ref }}^{2}\right\rangle \frac{2 \sigma_{w e+}^{2}}{\sigma_{w r}^{2}}\left(1+\frac{c}{\left\langle n_{\text {hit }}^{-1}\right\rangle}\right)$,

where $\sigma_{\text {wet }}$ is the stdev of the $(a+b) / 2$ white noise baseline errors, given in Table 3. Assuming Eq. (83) to hold for our maps, we have solved for $c$ for different $t_{\text {base }}$ (third column in Table 9). These numbers can be compared to Fig. 11 and Table 4.

Since the white noise reference baselines and the white noise baseline errors are uncorrelated, the full white noise baseline map rms is close to the rss of these two components. See Table 10 .

\subsubsection{Unmodeled $1 / f$ noise}

Most of the power in unmodeled $1 / f$ noise is in frequencies near $1 /\left(2 t_{\text {base }}\right)$. Therefore, for $\theta_{p} \ll \theta_{\text {base }}$ successive hits to the 
Table 10. rms of the white noise baseline maps.

\begin{tabular}{cccc}
\hline \hline$t_{\text {base }}$ & $\begin{array}{c}I_{\text {rms }} \\
(\mu \mathrm{K})\end{array}$ & $\begin{array}{c}Q_{\text {rms }} \\
(\mu \mathrm{K})\end{array}$ & $\begin{array}{c}U_{\text {rms }} \\
(\mu \mathrm{K})\end{array}$ \\
\hline $2.5 \mathrm{~s}$ & 6.729 & 9.303 & 9.580 \\
$15 \mathrm{~s}$ & 2.319 & 3.291 & 3.356 \\
$1 \mathrm{~min}$ & 1.181 & 1.678 & 1.715 \\
$1 \mathrm{~h}$ & 1.088 & 1.552 & 1.590 \\
\hline
\end{tabular}

Table 11. rms of the unmodeled $1 / f$ noise maps.

\begin{tabular}{llllll}
\hline \hline$t_{\text {base }}$ & $\begin{array}{l}\text { Eqs. ((56), (84)) } \\
(\mu \mathrm{K})\end{array}$ & $\begin{array}{l}\text { Eq. (84) } \\
(\mu \mathrm{K})\end{array}$ & $\begin{array}{l}I_{\text {rms }} \\
(\mu \mathrm{K})\end{array}$ & $\begin{array}{l}Q_{\text {rms }} \\
(\mu \mathrm{K})\end{array}$ & $\begin{array}{l}U_{\text {rms }} \\
(\mu \mathrm{K})\end{array}$ \\
\hline $7.5 \mathrm{~s}$ & 2.994 & 3.081 & 3.062 & 4.314 & 4.358 \\
$15 \mathrm{~s}$ & 3.727 & 3.901 & 3.852 & 5.450 & 5.526 \\
$1 \mathrm{~min}$ & 6.053 & 6.313 & 5.778 & 8.128 & 8.225 \\
$1 \mathrm{~h}$ & & & 9.357 & 13.02 & 13.178 \\
\hline
\end{tabular}

same pixel should be almost fully correlated. When $t_{\text {base }}$ is much below the spin period, hits from different spin periods should be almost uncorrelated. Thus we can estimate the unmodeled $1 / f$ noise map rms in the same manner as the white noise baseline map, as

$I_{\mathrm{rms}} \approx \sqrt{\left\langle\frac{\sum n^{2} f_{n}}{n_{\mathrm{hit}}}\right\rangle} \sigma_{u} \approx 0.025 \sigma_{u}$.

When $t_{\text {base }}$ is comparable to the spin period, there will be correlations (positive or negative) between hits from nearby spin periods. We compare Eq. (84) to the actual binned maps of unmodeled $1 / f$ noise in Table 11 . The second and third columns in the table are estimates for the $I \mathrm{rms}$ based on Eq. (84), with $\left\langle\frac{\sum n^{2} f_{n}}{n_{\text {hit }}}\right\rangle$ taken from Table 8 . The third column uses the actual $\sigma_{u}$, whereas the second column uses the analytical estimate (56). We see that for $t_{\text {base }}=1 \mathrm{~min}$, Eq. (84) is an overestimate, indicating that there are negative correlations between hits from nearby spin periods.

\subsubsection{Ideal scanning}

We define ideal scanning so that the pointings from the different scan circles of the same repointing period fall on top of each other, i.e., there is no nutation and the sampling is synchronized with the spin period. In this case, the part of the unmodeled $1 / f$ noise for long baselines ( $t_{\text {base }}$ a multiple of the spin period) that is modeled by $1 \mathrm{~min}$ baselines gets totally averaged out, so that the contribution from unmodeled $1 / f$ noise to residual maps would stay constant from $t_{\text {base }}=1 \mathrm{~min}$ to $t_{\text {base }}=1 \mathrm{~h}$. In our nonideal case, some of this noise leaks out, so that the unmodeled contribution rises slowly in this range also. See dashed red line in Fig. 26.

Likewise, for an ideal scanning, the white noise reference baselines make an equal contribution to the map for any baseline length that is an integer multiple of the spin period, and fits into the repointing period an integer number of times.

\subsubsection{Total noise}

From Fig. 26 we see that the two dominant contributions to the residual maps are the white noise reference baselines and the unmodeled $1 / f$ noise. For both of them we have analytical estimates, and both of them map from time domain to map domain in roughly the same way. Thus we get an analytical estimate for the residual $I$ map rms by multiplying the $\sqrt{\sigma_{w r}^{2}+\sigma_{u}^{2}}$ estimate from Eqs. (49) and (53) with $\sqrt{\left\langle\frac{\sum n^{2} f_{n}}{n_{\text {hit }}}\right\rangle} \approx 0.025$ for $t_{\text {base }} \leq$ 1 min. When $\alpha>1$ and $t_{\text {base }} \gg t_{\text {sample }}$, so that $\left(f_{x} / f_{\mathrm{c}}\right)^{\alpha} \ll 1$, we have

$$
\begin{aligned}
I_{\mathrm{rms}}^{2} \approx & \sigma^{2}\left\langle\left\langle\sum n^{2} f_{n}\right\rangle\right\rangle\left\langle n_{\text {hit }}^{-1}\right\rangle\left[\frac{1}{f_{\text {sample }} t_{\text {base }}}\right. \\
& \left.+\left(\frac{2 f_{\mathrm{k}}}{f_{\text {sample }}}\right)\left(2 f_{\mathrm{k}} t_{\text {base }}\right)^{\alpha-1}\left(\frac{4}{(5-\alpha)(\alpha-1)}\right)\right] .
\end{aligned}
$$

This gives for our case $\left(\sigma=2700 \mu \mathrm{K},\left\langle\left\langle\sum n^{2} f_{n}\right\rangle\right\rangle=1.52, n_{\text {hit }}^{-1}=\right.$ $\left.0.000418, f_{\text {sample }}=76.8 \mathrm{~Hz}, \alpha=1.7\right)$,

$I_{\text {rms }}^{2} \approx 60.3 t_{\text {base }}^{-1}+2.08 t_{\text {base }}^{0.7}$

where $t_{\text {base }}$ is given in seconds. (For $t_{\text {base }} \geq 1 \mathrm{~min}$, our analytical treatment can just estimate that the map rms should stay constant from $t_{\text {base }}=1 \mathrm{~min}$ to $t_{\text {base }}=1 \mathrm{~h}$.)

However, this estimate is not as good as in the time domain, since the importance of the neglected components, the baseline errors, has grown dramatically when going from the time domain to the map domain. See Fig. 26. Since these components rise towards shorter baselines, the residual map rms is minimized at a somewhat larger $t_{\text {base }}$ than Eq. (58) gives.

\subsection{Pixelization noise and signal baselines}

For pixelization noise, already the reference baselines are strongly correlated (see Fig. 23) and therefore their map rms cannot be estimated like for white noise reference baselines and unmodeled $1 / f$ noise. Due to these correlations their impact in the map level is significantly larger than their small variance in the time level (see Table 7) would indicate. Instead, for both the pixelization noise reference baselines and the signal baseline error, the situation is similar to white noise and $1 / f$ noise baseline error.

For the residual $1 / f$ and white noise baselines, the $Q$ and $U$ maps look the same as the $I$ maps, just with a factor $\sim \sqrt{2}$ larger amplitude, since they originate from the same timedomain noise, which is independent for each detector.

For the signal baselines (see Fig. 32) the situation is, however, different, since they originate from the signal, where $Q$ and $U$ are much smaller than $I$. We also note that $Q$ is much larger than $U$, although they are of same magnitude in the signal. This is related to the coordinate dependence of the definition of the Stokes parameters $Q$ and $U$ together with a combination of factors in our study. First, the signal contains only $E$ mode polarization, which means that $Q$ has structures along the coordinate lines, whereas $U$ has structures oriented $45^{\circ}$ from them. Second, we are using ecliptic coordinates, and we have employed a scanning strategy, where the scanning goes almost parallel to the lines of longitude for a large part of the sky. The signal baselines originate from the signal gradients within pixels. For a signal structure oriented along the scanning direction, the signal gradient structure remains similar for a sequence of pixels along the scanning. Thus the measurement differences between different scans through these pixels are similar for a sequence of pixels, favoring their misinterpretation as noise baselines.

To verify the effect of the coordinate system, we redid the $t_{\text {base }}=1 \mathrm{~h}$ and $1 \mathrm{~min}$ cases using galactic coordinates. See Table 12 ( $\mathrm{E}=$ ecliptic, $\mathrm{G}=$ galactic). Note that the map rms is given in $\mathrm{nK}$ (not in $\mu \mathrm{K}$, like the other tables). $P$ stands for 

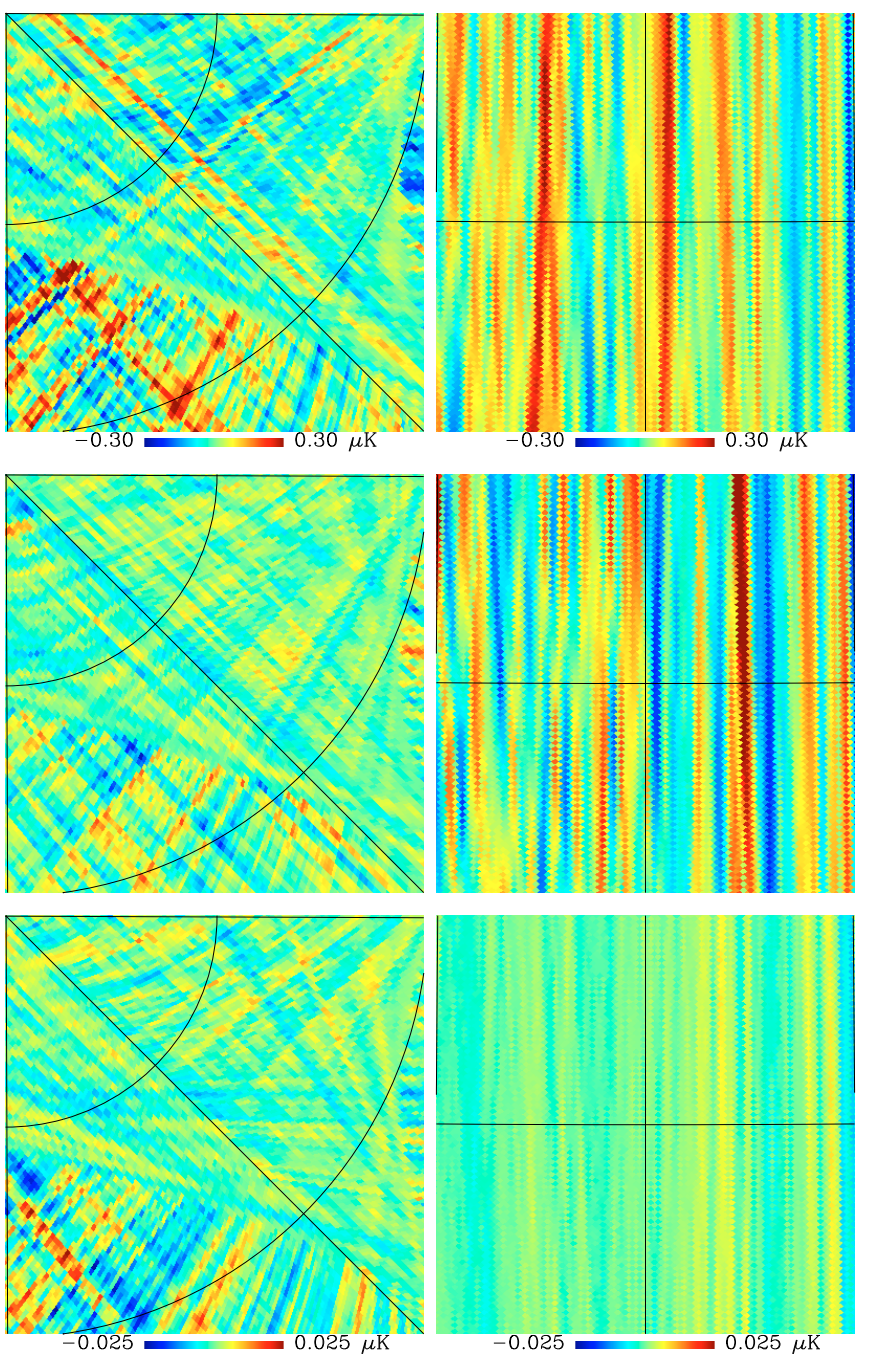

Fig. 32. Signal baseline $I$ (top), $Q$ (middle), and $U$ (bottom) maps -BFAs $\left(t_{\text {base }}=1 \mathrm{~min}\right)$ for the two $10^{\circ} \times 10^{\circ}$ regions.

Table 12. Effect of the coordinate system on the signal baseline maps.

\begin{tabular}{cccccc}
\hline \hline$t_{\text {base }}$ & Coord. & $\begin{array}{c}I \mathrm{rms} \\
(\mathrm{nK})\end{array}$ & $\begin{array}{c}Q \mathrm{rms} \\
(\mathrm{nK})\end{array}$ & $\begin{array}{c}U \mathrm{rms} \\
(\mathrm{nK})\end{array}$ & $\begin{array}{c}P \mathrm{rms} \\
(\mathrm{nK})\end{array}$ \\
\hline $1 \mathrm{~min}$ & $\mathrm{E}$ & 123.3 & 9.4 & 4.5 & 10.4 \\
$1 \mathrm{~min}$ & $\mathrm{G}$ & 115.9 & 6.8 & 7.9 & 10.4 \\
$1 \mathrm{~h}$ & $\mathrm{E}$ & 124.0 & 9.4 & 4.6 & 10.5 \\
$1 \mathrm{~h}$ & $\mathrm{G}$ & 116.5 & 6.9 & 7.9 & 10.5 \\
\hline
\end{tabular}

$\sqrt{Q^{2}+U^{2}}$. We see that the asymmetry between $Q$ and $U$ largely disappears, but the total polarization signal residual is not much affected. For the temperature residual we see a small improvement. This is partly explained by the reduction of the signal baseline variance, seen in Table 7.

\subsection{Residuals at different angular scales}

The residual map rms alone is a poor measure of the quality of the output map. Since the nature of the residual (see Figs. 29 and 30) is different for different baseline lengths, we need to look at the structure of the different map residuals in more detail.

For long baselines, the residual mostly comes from the part of the $1 / f$ noise that cannot be modeled with baselines, and appears mostly at very small angular scales on the map, near the pixel scale; whereas for shorter baselines it comes from unwanted baselines, which appear as larger scale structures. This can be seen from Fig. 27, where we have smoothed the residual map with the detector beam, before taking the rms. This smoothing almost erases the difference between the $1 / f$ residuals for baseline lengths from $t_{\text {base }}=1 \mathrm{~min}$ to $1 \mathrm{~h}$. This is because the 1 min scanning circles fall almost on top of each other during the $1 \mathrm{~h}$ repointing period, and the width (due to nutation) of the ring on the sky traced by the beam center during a repointing period is less than the beam width. Beam-smoothing has much less effect on the white noise baseline map and the signal baseline map. Baseline lengths $t_{\text {base }}=10 \mathrm{~s}$ to $15 \mathrm{~s}$ still give the smallest total residuals, but the difference from longer baselines is much reduced by beam-smoothing. Since residuals at larger scales are for most purposes more harmful than sub-beam residuals on the map, we conclude that it is better to choose a somewhat longer baseline than what would minimize the residual map rms.

\subsection{Angular power spectra of map residuals}

Since we are considering full-sky maps, their angular power spectra $C_{\ell}^{X Y}$ can be calculated directly from them (we used anafast of the HEALPix package).

We plot the angular power spectra of the residual maps in Figs. 33 and 34 for different baseline lengths. It is clear that baselines shorter than $t_{\text {base }}=10 \mathrm{~s}$, lead to more large scale structure in the residuals. Long baselines lead to a high- $\ell$ tail in the residual that appears much like white noise (flat $C_{\ell}$ ).

In Figs. 35 and 36 we show angular power spectra $C_{\ell}^{T T}$ and $C_{\ell}^{E E}$ of different map components for the cases $t_{\text {base }}=1 \mathrm{~h}$, $1 \mathrm{~min}, 15 \mathrm{~s}$, and $5 \mathrm{~s}$.

For baselines that are multiples of the spin period (1 min and $1 \mathrm{~h}$ ) we see the characteristic even-odd variation in the $C_{\ell}$ of the baseline components. If the scanning circles had the full $90^{\circ}$ radius, they would contribute only to the even multipoles. In our case the circle radius is $87.77^{\circ}=90^{\circ}-2.23^{\circ}$, and therefore we see a beat pattern, where the maximum even-odd multipole difference occurs at multipoles $\ell$ that are near multiples of $90^{\circ} / 2.23^{\circ}=40.4$. For the low $\ell$ of the unmodeled $1 / f$ contribution we see the opposite pattern, since the unmodeled $1 / f$ contains mostly frequencies which vary just over those timescales over which the baseline contributions are constant.

The angular power spectrum of full-circle uncorrelated baselines goes as

$C_{\ell} \propto \frac{1}{2 \ell+1}$

(Eftstathiou 2005). Therefore we have plotted $(2 \ell+1) C_{\ell} / \pi$ in Figs. 35 and 36. We see that Eq. (87) indeed holds well for white noise reference baselines; even for $t_{\text {base }} \ll 1 / f_{\text {spin }}$, although these have less power at the lowest $\ell$. Although the unmodeled $1 / f$ contribution does not consist of constant baselines, the correlations of the parts between different baseline segments are weak (nonexistent for $t_{\text {base }} \ll 1 / f_{\text {spin }}$ ). Therefore Eq. (87) holds fairly well for the unmodeled $1 / f$ also; except at low $\ell$ for short baselines, where there is a lack of power since the unmodeled $1 / f$ varies more rapidly along the scan path; and for high $\ell$ for $t_{\text {base }} \gg 1 / f_{\text {spin }}$, which have excess power at high $\ell$, related to imperfect superposition of the different scan circles of the same ring, mainly due to nutation.

The other contributions have different angular scale dependencies, related to the correlations between baselines. We see that the baseline error components have steeper spectra than the 

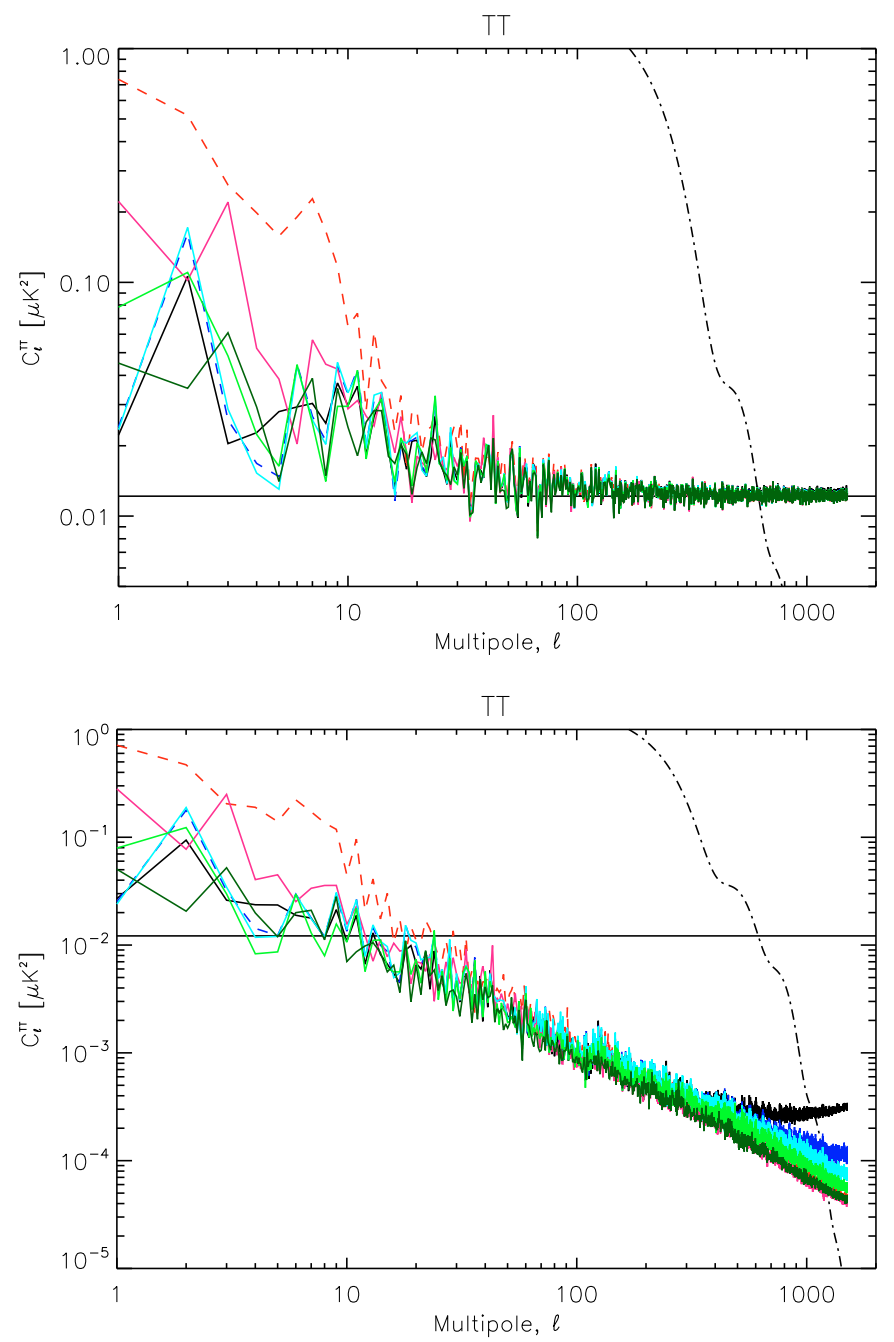

Fig. 33. Angular power spectra of the residual temperature maps for different baseline lengths: $1 \mathrm{~h}$ (black), $4 \mathrm{~min}$ (blue dashed), $1 \mathrm{~min}$ (light blue), $30 \mathrm{~s}$ (light green), $15 \mathrm{~s}$ (green), $7.5 \mathrm{~s}$ (pink), and $5 \mathrm{~s}$ (red dashed). The black horizontal line is the white noise level and the black dotdashed line is the theoretical CMB input spectrum smoothed with the beam and pixel window functions. Top: residual map including white noise, $\boldsymbol{m}_{\text {out }}-\mathbf{B} \boldsymbol{s}$. Bottom: residual map $\boldsymbol{m}_{\text {out }}-\mathbf{B} \boldsymbol{s}-\mathbf{B} \boldsymbol{w}$ (binned white noise subtracted).

reference baseline and unmodeled $1 / f$ contributions. This makes them important at large scales (low multipoles), where they are comparable or even stronger than the white noise reference baseline and unmodeled $1 / f$ components, which dominate at high $\ell$ and contribute most to the residual map rms. If one considers just the signal baselines, the baseline error completely dominates over the reference baselines for short $t_{\text {base }}$ and low $\ell$.

For long baselines $(\sim 1 \mathrm{~min}$ or longer), the unmodeled $1 / f$ noise dominates the residuals for $\ell>10$, but the $1 / f$ baseline error contribution can be comparable for $\ell<10$. For shorter baselines, the white noise baselines become more important.

The $C_{\ell}^{B B}$ spectra of different residual components look qualitatively like $C_{\ell}^{E E}$, except for the signal baseline components, which have less power, reflecting the lack of B-mode signal in the input. Therefore we have not plotted the $C_{\ell}^{B B}$ spectra, except for these signal baseline components, which we have included in Fig. 36 along with the $C_{\ell}^{E E}$ spectra.
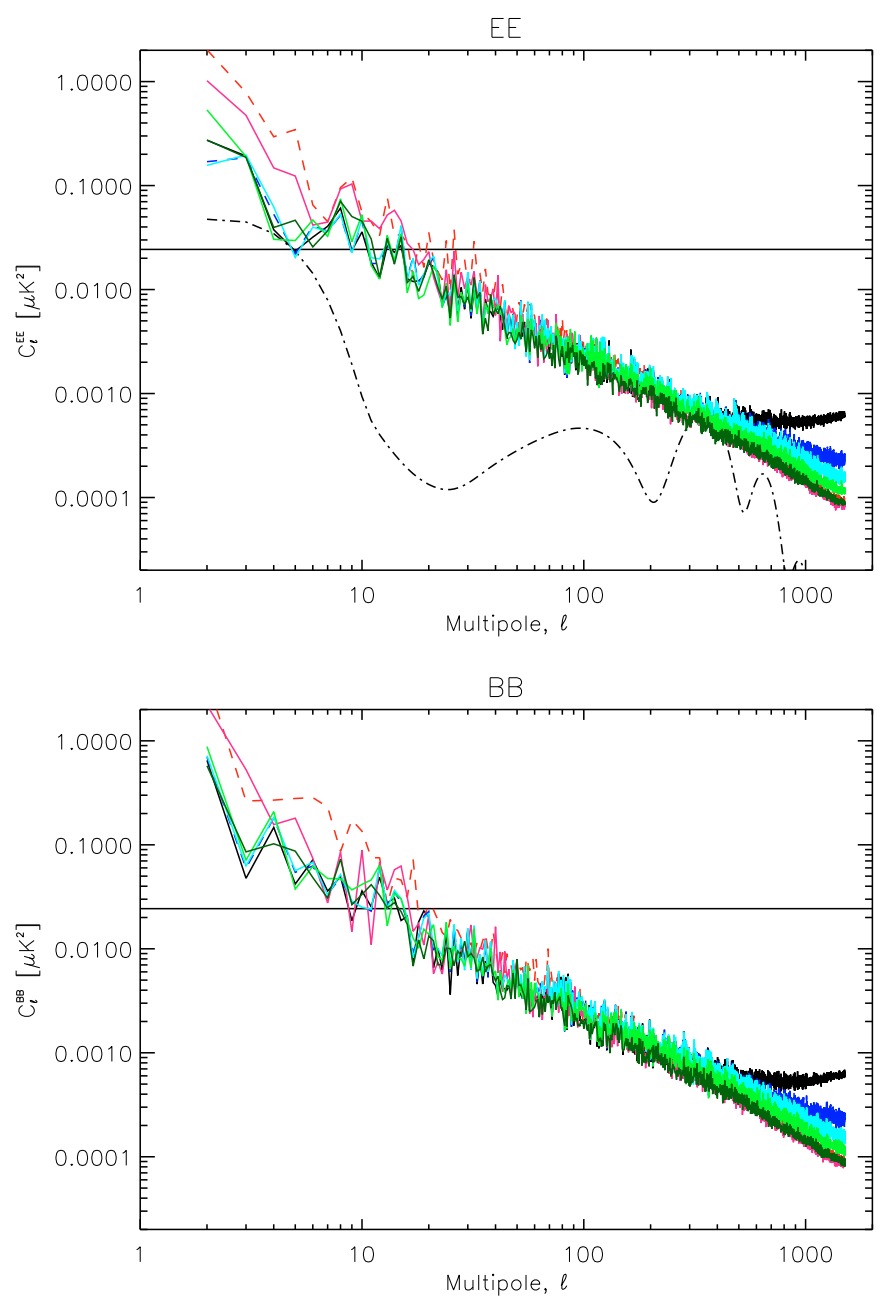

Fig. 34. Same as Fig. 33 bottom panel, but for the polarization $E$ and $B$ mode spectra.

Because of large $\ell$-to- $\ell$ variations in the residual $C_{\ell}$, these plots are difficult to read. Therefore we also plot (Fig. 37) square roots of the cumulative angular power spectra,

$\sqrt{\sum_{\ell^{\prime}=1}^{\ell} \frac{2 \ell^{\prime}+1}{4 \pi} C_{\ell^{\prime}}^{T T}}, \quad \sqrt{\sum_{\ell^{\prime}=2}^{\ell} \frac{2 \ell^{\prime}+1}{4 \pi}\left(C_{\ell^{\prime}}^{E E}+C_{\ell^{\prime}}^{B B}\right)}$,

which give the total contribution to the residual $I$ and $P$ map rms from multipoles up to $\ell$, and

$\sqrt{\sum_{\ell^{\prime}=2}^{\ell} \frac{2 \ell^{\prime}+1}{4 \pi}\left|C_{\ell^{\prime}}^{T E}\right|}$

The beam fwhm $\theta=12.68^{\prime}$ corresponds to multipole $\ell=$ $180^{\circ} / \theta \sim 850$, so we are more concerned about the behavior up to this $\ell$ than above it.

Figure 37 provides probably the most concise meaningful comparison of the quality of maps vs. baseline length. The $t_{\text {base }}=$ $15 \mathrm{~s}$ case appears the best in terms of cumulative residual power in the map for the relevant multipoles. Although $t_{\text {base }}=10 \mathrm{~s}$ produces a smaller residual map rms, it is only because of its small sub-beam-scale residuals. Interestingly, the $t_{\text {base }}=1 \mathrm{~h}$ baseline seems to be the best for minimizing residual temperaturepolarization correlations at intermediate scales. It is also better overall than the $t_{\text {base }}=1 \min$ and 4 min cases for $\ell<300$. 


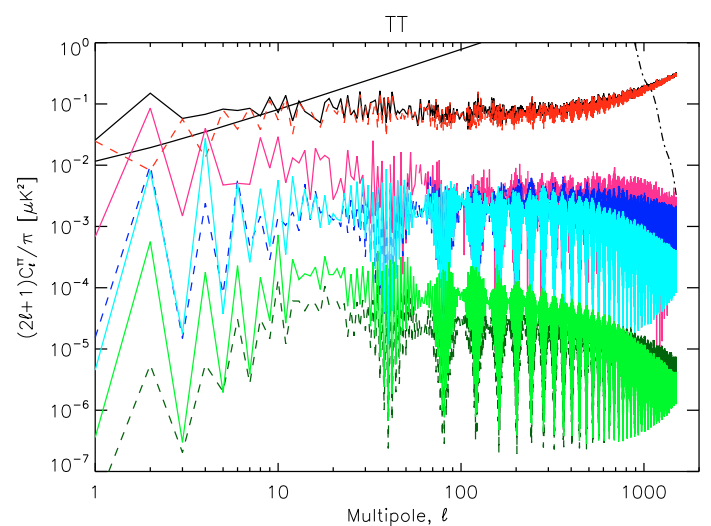

$\mathrm{TT}$
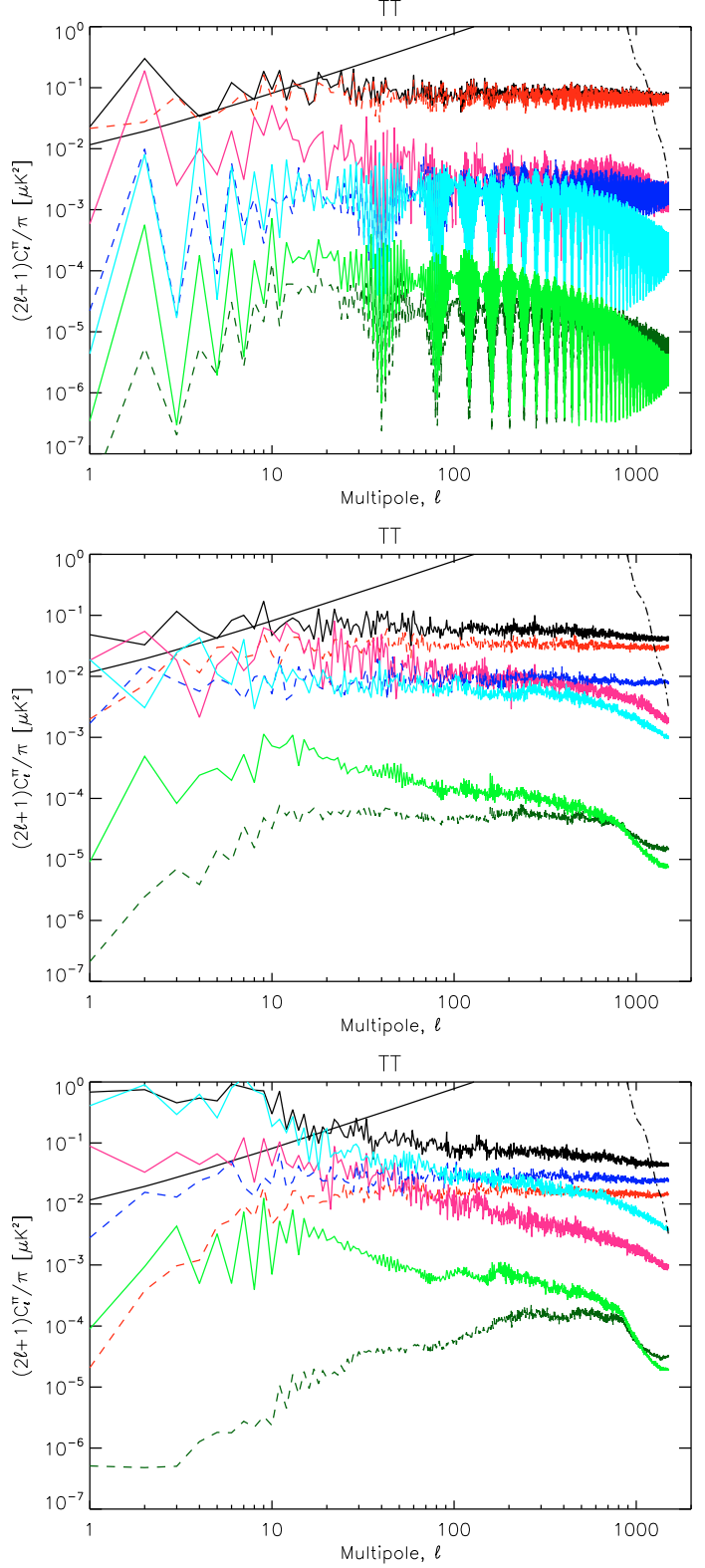

Fig. 35. Angular temperature power spectra $C_{\ell}^{T T}$ of different map components for four different baseline lengths: $1 \mathrm{~h}$ (top panel), $1 \mathrm{~min}, 15 \mathrm{~s}$, and $5 \mathrm{~s}$ (bottom panel). Solid black: residual map (with binned white noise subtracted). This can be split into the following six components: red dashed: unmodeled $1 / f$ noise. Pink: additional effect of $1 / f$ baseline error. Blue dashed: white noise reference baselines. Light blue: white noise baseline error. Green dashed: reference baselines of pixelization noise. Light green: additional effect of signal baseline error. The smooth black curve is the white noise level.
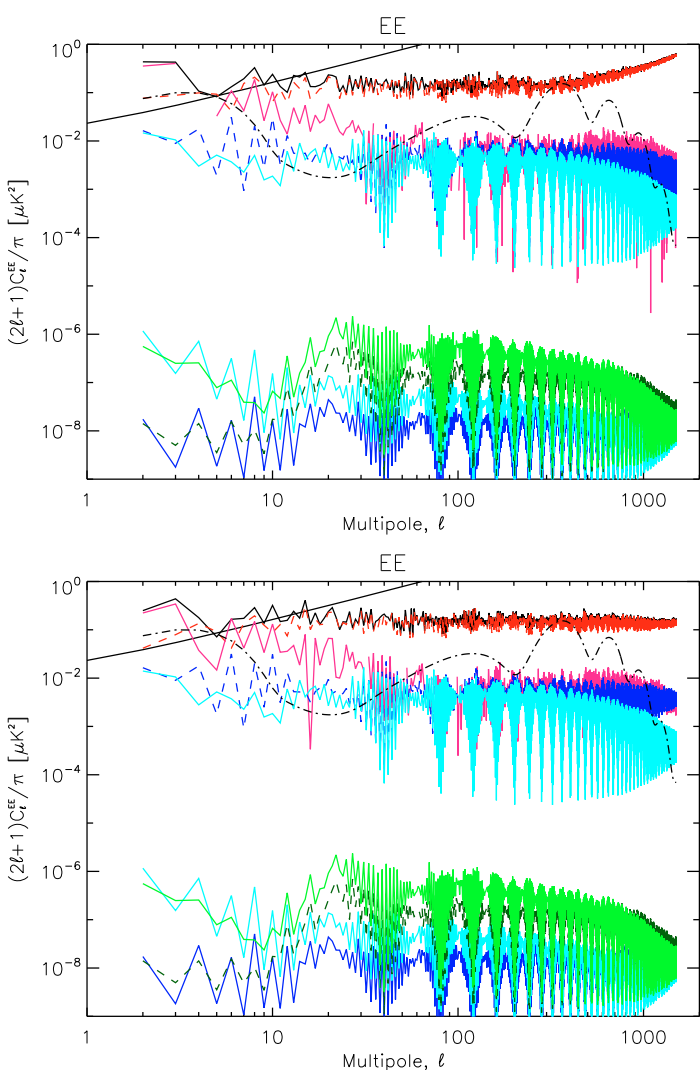

EE
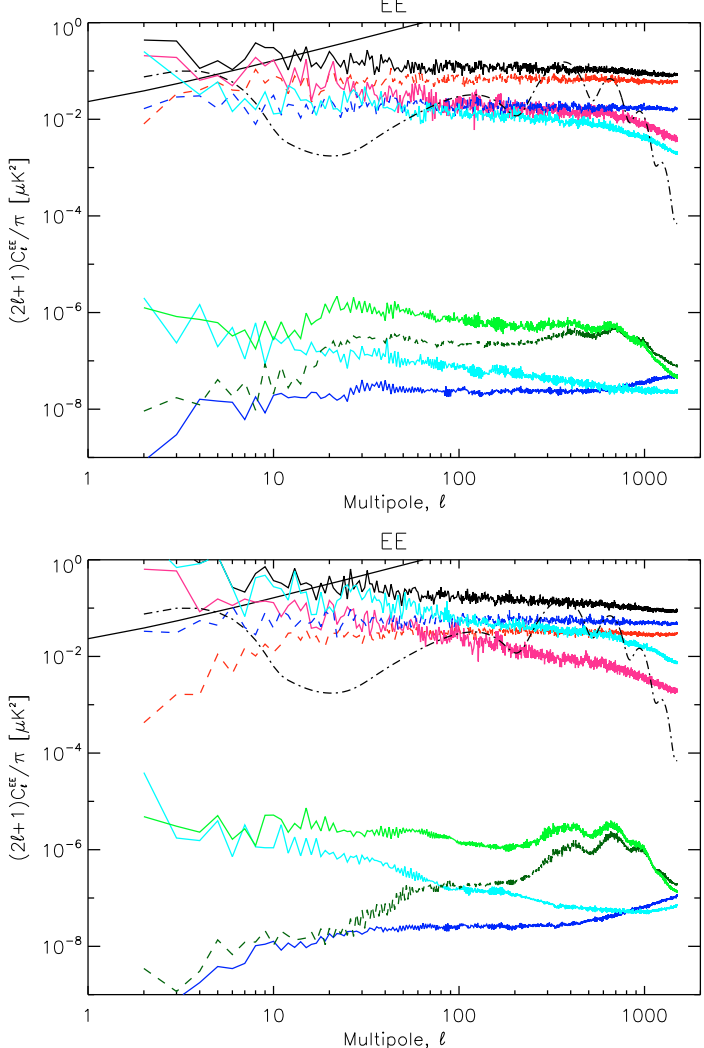

Fig. 36. Same as Fig. 35 but for the $E$ mode polarization spectrum $C_{\ell}^{E E}$. We also show the two signal baseline contributions of the $B$ mode polarization spectrum $C_{\ell}^{B B}$. They are the lower blue curves: blue: reference baselines of pixelization noise. Light blue: additional effect of signal baseline error. 

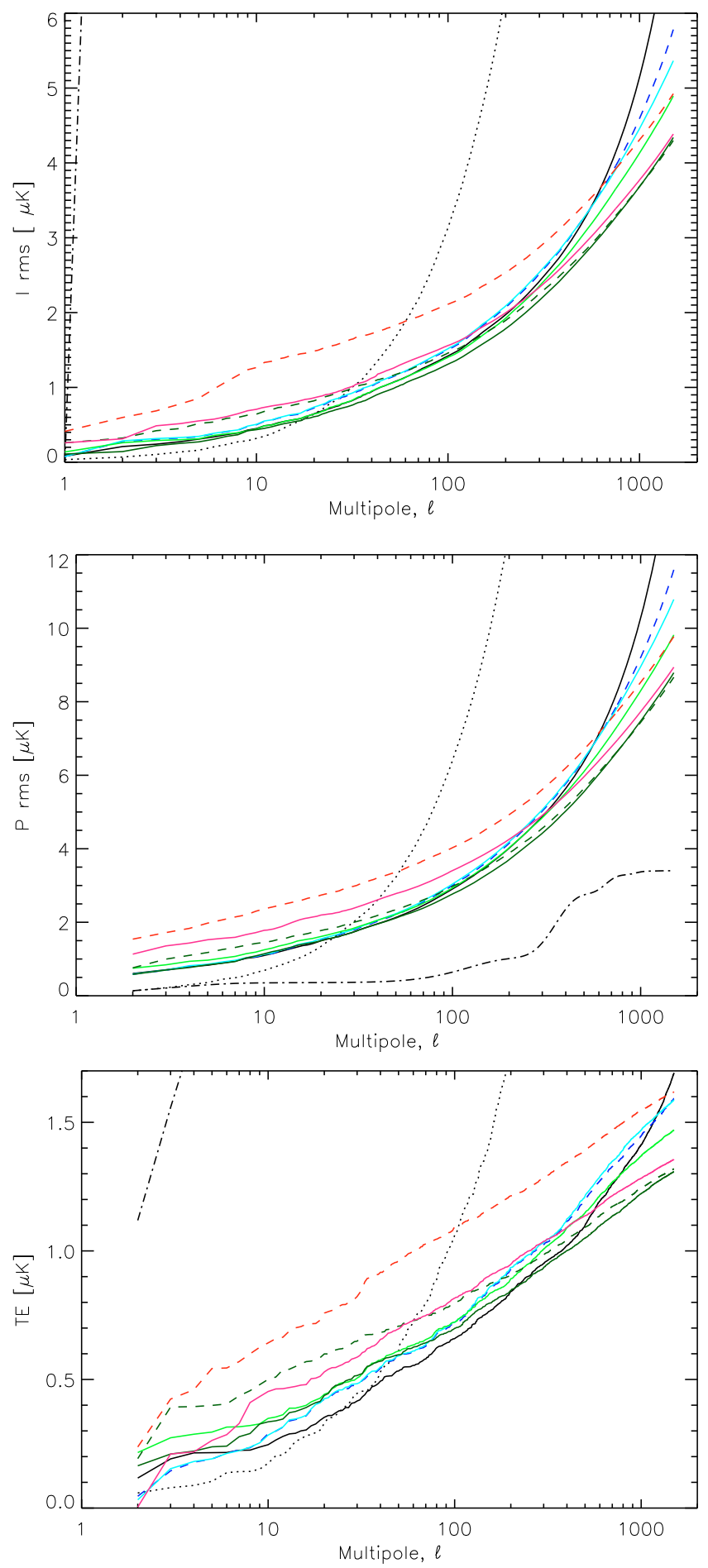

Fig. 37. Square roots of the cumulative angular power spectra (see Eqs. (88) and (89)) for the residual temperature (top panel) and polarization (middle) maps and TE correlation (bottom), up to a given multipole $\ell$, plotted as a function of $\ell$ for different baseline lengths: $1 \mathrm{~h}$ (black), 4 min (blue dashed), $1 \mathrm{~min}$ (light blue), $30 \mathrm{~s}$ (light green), $15 \mathrm{~s}$ (green), $10 \mathrm{~s}$ (green dashed), $7.5 \mathrm{~s}$ (pink), and $5 \mathrm{~s}$ (red dashed). The black dot-dashed line corresponds to the theoretical input CMB spectrum. The dotted line is calculated from the binned white noise map.

If one is only interested in large-scale features (low $\ell$ ) there are no big differences between any of the baseline lengths from $15 \mathrm{~s}$ to $1 \mathrm{~h}$, but $10 \mathrm{~s}$ or less should be avoided. For the lowest multipoles there is some randomness in the results, since we studied only one noise realization, so one should not try to draw conclusions from the small differences seen there for $t_{\text {base }}=15 \mathrm{~s}$ to $1 \mathrm{~h}$. (The noise residuals, for the cases $t_{\text {base }}=1 \mathrm{~min}$ and $t_{\text {base }}=1.25 \mathrm{~s}$ with noise prior, have been studied via Monte Carlo in Keskitalo et al. 2009.)

The signal baseline contributions appear a minor effect at all scales. In this study the signal contained only the CMB. In reality, the gradients in the signal are often dominated by foregrounds, and therefore the signal baseline effect is larger. Foreground signals are considered in Keihänen et al. (2009). Foregrounds were also included in the map-making studies of Ashdown et al. (2007b, 2009), and especially in the former there was a detailed study on the signal baseline contribution and how it could be minimized.

Map residuals influence the precision at which we are able to determine the angular power spectrum of the CMB map. We can subtract the expectation value of the $C_{\ell}$ of the residual from the map spectrum, but individual realizations deviate from this expectation value, leading to an error in the CMB $C_{\ell}$ estimate. A multipole $\ell$ of a map has at best $2 \ell+1$ degrees of freedom. Statistically isotropic signals (e.g. CMB or a white noise map of uniform pixel variance) have these degrees of freedom. Deviations from the statistical isotropy may lead to correlations in the $m$-modes of a multipole which in turn decreases the degrees of freedom and therefore increase the error in $C_{\ell}$. Fortunately the strongest correlations of the map residuals occur nearly along the ecliptic meridians. Correlations along meridians do not lead to correlations in the $m$-modes. Therefore we can expect that the $m$-mode couplings due to map residuals are weak and the excess spectrum error is small. For now, we did not investigate these errors any further, but decided to leave this for future studies.

\subsection{Low multipoles of $I, Q$, and $U$ maps}

For cosmological purposes, one calculates the $C_{\ell}^{T T}, C_{\ell}^{T E}, C_{\ell}^{E E}$, and $C_{\ell}^{B B}$ angular power spectra of the output maps, which represent the fundamental properties of the temperature and polarization field, and are coordinate independent. However, for analyzing residual map structure, it may be more intuitive to consider the $Q$ and $U$ maps as two separate maps of a scalar quantity, and calculate their ordinary (spin-0) angular power spectra.

The $Q$ and $U$ are given in terms of the $a_{\ell m}^{E}$ and $a_{\ell m}^{B}$ as (Zaldarriaga \& Seljak 1997)

$Q+i U=-\sum\left(a_{\ell m}^{E}+i a_{\ell m}^{B}\right)_{2} Y_{\ell}^{m}$,

where the

${ }_{2} Y_{\ell}^{m}(\theta, \phi) \equiv \sqrt{\frac{2 \ell+1}{4 \pi}} \mathrm{e}^{i m \phi} d_{m,-2}^{\ell}(\theta)$

are the spin-2 spherical harmonics (Newman \& Penrose 1966; Goldberg et al. 1967) and the $d_{m, m^{\prime}}^{\ell}(\theta)$ are the Wigner $d$-functions (we follow Varshalovich et al. 1988), which are real.

Thus the monopoles $\bar{Q}$ and $\bar{U}$ of the $Q$ and $U$ maps are given by

$\bar{Q}+i \bar{U}=-\sum\left(a_{\ell m}^{E}+i a_{\ell m}^{B}\right)_{2} \bar{Y}_{\ell}^{m}$,

where

$$
\begin{aligned}
{ }_{2} \bar{Y}_{\ell}^{m} & \equiv \frac{1}{4 \pi} \int{ }_{2} Y_{\ell}^{m}(\theta, \phi) \mathrm{d} \Omega \\
& =\delta_{m 0} \sqrt{\frac{2 \ell+1}{16 \pi}} \int_{0}^{\pi} d_{0,-2}^{\ell}(\theta) \sin \theta \mathrm{d} \theta,
\end{aligned}
$$




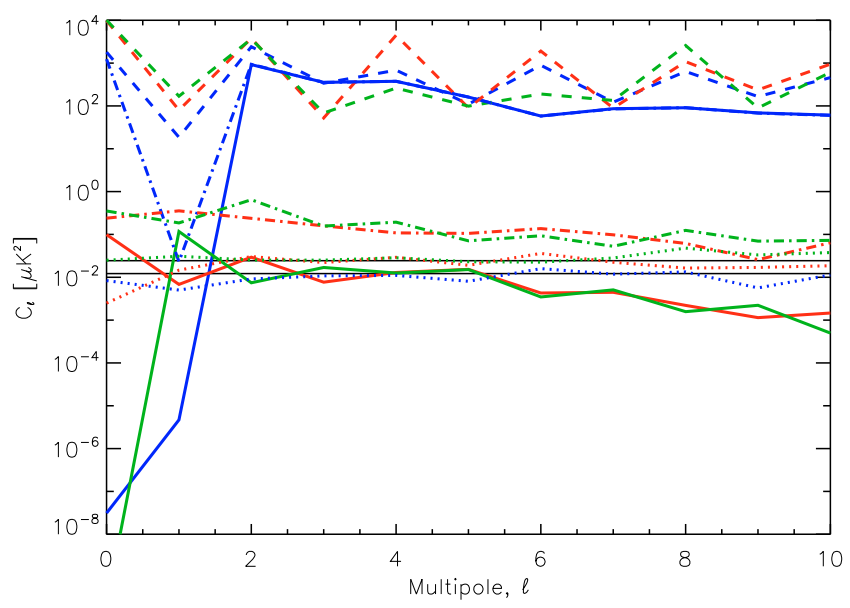

Fig. 38. Effect of destriping on the low multipoles of $I$ (blue), $Q$ (red), and $U$ (green) maps. The solid lines give the multipoles of the binned signal (CMB) map, where the monopoles of $I$ and $U$, and the dipole of $I$ should ideally vanish in our case. The dashed lines are for the naive binned map including the noise, and the dot-dashed lines are for the destriped $\left(t_{\text {base }}=1 \mathrm{~min}\right)$ map. The dotted lines are for the binned white noise map. The horizontal black lines give the expected white noise levels.

which are real. Thus the $Q$ and $U$ monopoles are

$\bar{Q}=-\sum_{\ell} a_{\ell 02}^{E} \bar{Y}_{\ell}^{0} \quad$ and $\quad \bar{U}=-\sum_{\ell} a_{\ell 0}^{B}{ }_{2} \bar{Y}_{\ell}^{0}$.

Since in our case the input spectrum contained no $B$ mode, the monopole vanishes in the input $U$ map.

Figure 38 illustrates the effect of destriping on the $I, Q$, and $U$ maps. Destriping is not able to remove the noise monopole of the $I$ map (the monopole does change but does not become small), but the noise monopoles of $Q$ and $U$ maps are removed, about equally well as the other low multipoles.

The low multipoles of the residual maps are shown in Fig. 39, divided by the white noise level. We see that the residuals at lowest multipoles are larger than the white noise level. The baseline length does not make a large difference for these low multipoles, except that too short baselines (less than $15 \mathrm{~s}$, not included in Fig. 39) should be avoided.

\section{Effect of noise knee frequency}

The importance of the correlated $1 / f$ noise depends on its amplitude and spectrum. In this paper we do not consider the effect of possible spectral features in the noise, and we have parameterized the noise just by the $1 / f$ slope $-\alpha$, knee frequency $f_{\mathrm{k}}$, and white noise level. Since we produced the simulated $1 / f$ noise separately from the white noise, we can change $f_{\mathrm{k}}$ simply by multiplying the $1 / f$ part by

$r_{1 \rightarrow 2}=\left(\frac{f_{k, 2}}{f_{k, 1}}\right)^{\alpha / 2}$.

This will change the residual $1 / f$ contribution to the residual map by the same factor, while the white noise baseline and signal baseline contributions are unaffected (unless one changes to a different $\left.t_{\text {base }}\right)$. For angular power spectra the $1 / f$ contributions are rescaled by the square of this factor. See Fig. 40, where we have plotted the residual map rms as a function of $t_{\text {base }}$ for $f_{\mathrm{k}}=100 \mathrm{mHz}$ and $25 \mathrm{mHz}$, besides the case of $f_{\mathrm{k}}=50 \mathrm{mHz}$,
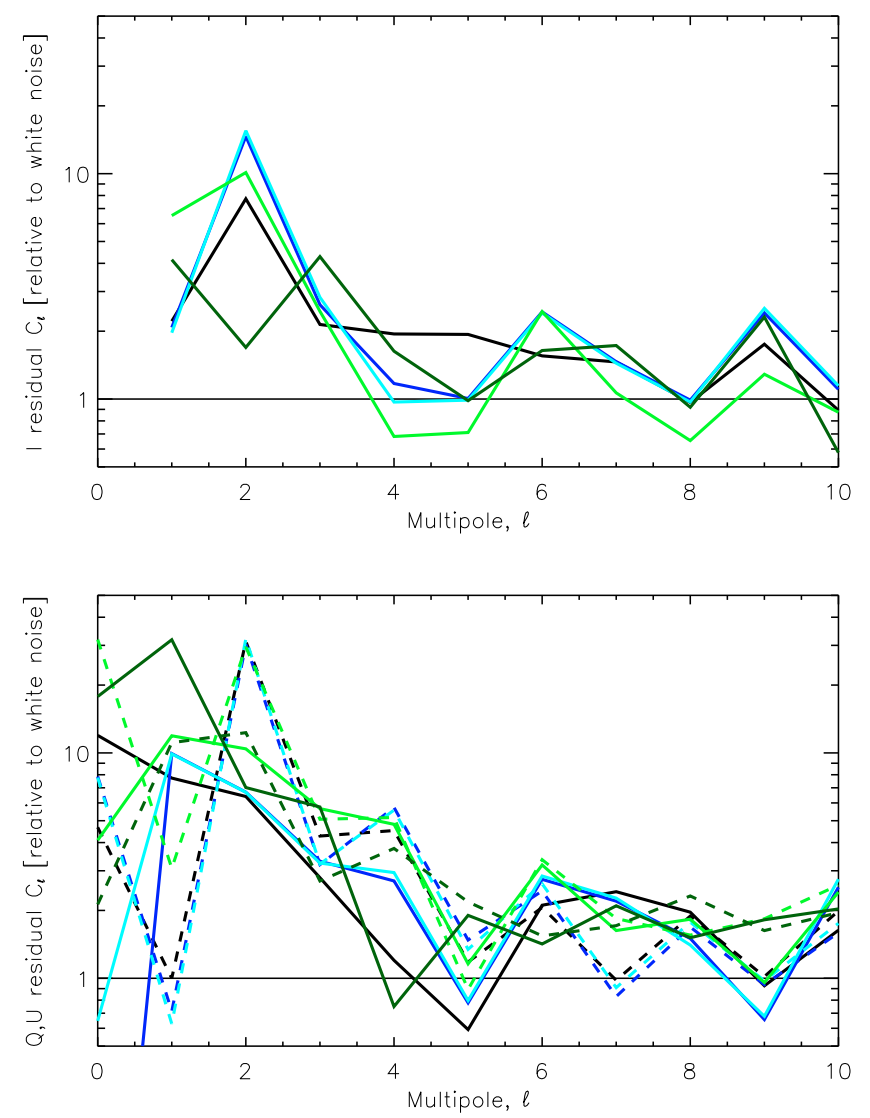

Fig. 39. The $I, Q$, and $U$ low multipoles of the residual maps (with binned white noise subtracted) for different baseline lengths: $1 \mathrm{~h}$ (black), $4 \mathrm{~min}$ (blue), $1 \mathrm{~min}$ (light blue), $30 \mathrm{~s}$ (light green), and $15 \mathrm{~s}$ (dark green). For this plot they are divided by the white noise level. The top panel shows $I$, the bottom panel $Q$ (solid) and $U$ (dashed).

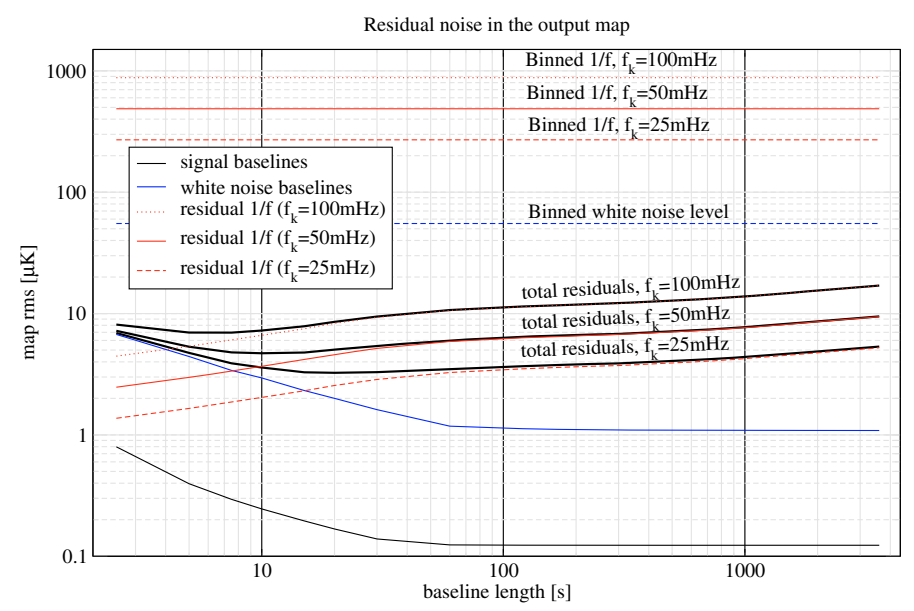

Fig. 40. Effect of knee frequency on the residual noise in the $I$ map.

which we have so far considered. A higher $f_{\mathrm{k}}$ favors shorter baselines, since the stronger $1 / f$ noise at relatively high frequencies needs to be modeled better. Reducing the amount of correlated noise (and hence the knee frequency) causes the white noise baseline contribution to exceed residual $1 / f$ contribution at longer baselines. Therefore a lower $f_{\mathrm{k}}$ favors longer baselines, as the white noise and signal baselines are relatively more important. For $f_{\mathrm{k}} \sim 10 \mathrm{mHz}$ the minimum rms would move to $t_{\text {base }} \sim 1$ min. 

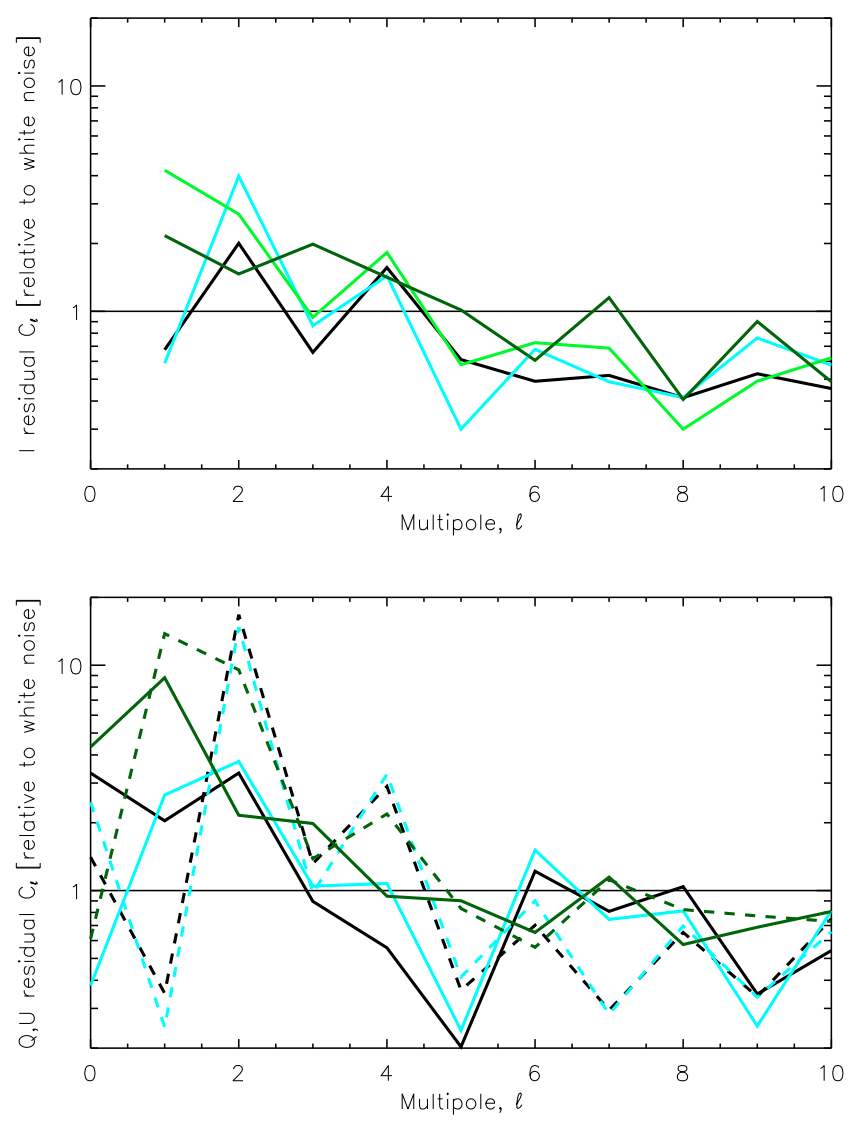

Fig. 41. Same as Fig. 39, but for a lower knee frequency $f_{\mathrm{k}}=25 \mathrm{mHz}$ (and a smaller selection of baseline lengths).

In Fig. 40 we show also the rms of the binned $1 / f$ noise map. As we lower $f_{\mathrm{k}}$ it moves down. For very small $f_{\mathrm{k}}$ it would fall below the white noise baseline rms. At this point simple binning would produce a better result than destriping. For our simulated $1 / f$ noise, and for long baselines, this would happen at the extremely low $f_{\mathrm{k}}=21.6 \mu \mathrm{Hz}$. It should be noted however, that in our case the binned $1 / f \mathrm{rms}$ is heavily dominated by the lowest frequencies, and in other cases (smaller slope $\alpha$, larger $f_{\min }$ ) the relevant $1 / f$ rms could be smaller for a given $f_{\mathrm{k}}$, so that simple binning could become superior already at a higher $f_{\mathrm{k}}$.

In Fig. 41 we show the low multipoles of the residual $I, Q$, and $U$ maps recalculated for $f_{\mathrm{k}}=25 \mathrm{mHz}$.

\section{Effect of noise prior}

Although short baselines can potentially model correlated noise better, they fail because of the large random amplitudes they pick from white noise. This can be remedied by applying prior information on the noise spectrum to prevent too large differences between amplitudes of nearby baselines. This is discussed in detail in Keihänen at al. (2009), but we give a short preview of the results here. See Figs. 42-44. The noise prior has little effect for $t_{\text {base }} \gg 1 / f_{\mathrm{k}}$, but for short baselines the effect is dramatic. We note that now the results keep improving as the baseline is shortened, at least until $t_{\text {base }}=2.5 \mathrm{~s}$, the shortest we tried. (Using very short baselines with the noise prior makes the code more resource intensive.) For the very lowest multipoles, the results with the short baselines do not, however, become much better than the ones obtained with longer baselines (with or without noise filter). Compare Figs. 44 to 39 and 41.

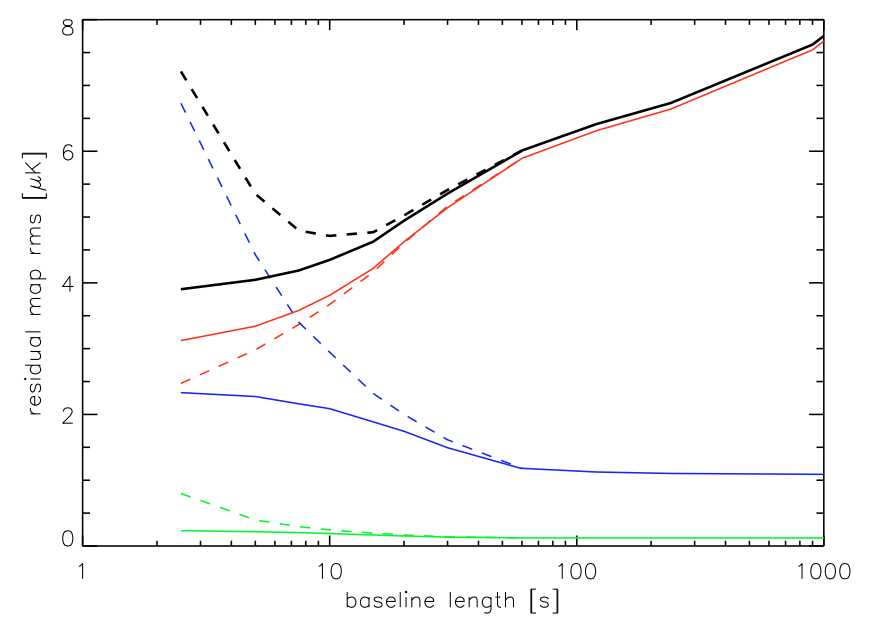

Fig. 42. Same as the bottom panel of Fig. 25, but the solid lines correspond to using a noise prior. The dashed lines are from Fig. 25, and correspond to the case without noise prior. Black lines show the rms of the residual $I$ map without the binned white noise component. Red is for the unmodeled $1 / f$ map, blue for the white noise baseline map, and green for the signal baseline map. The noise prior has practically no effect for $t_{\text {base }} \geq 1 \mathrm{~min}$.

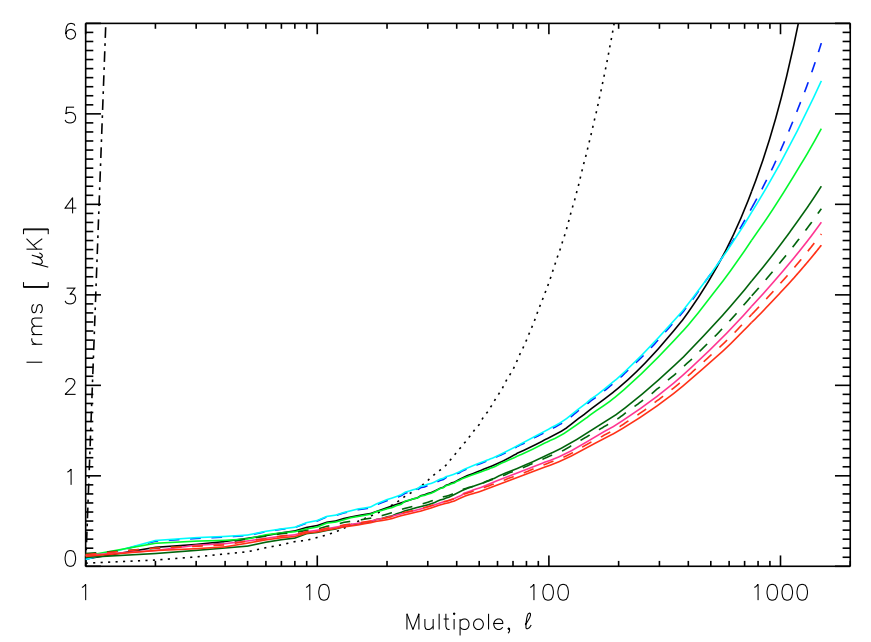

Fig. 43. Same as top panel of Fig. 37 but for the case of using the noise prior. The colors and linestyles are the same as in Fig. 37, except we have added the $t_{\text {base }}=2.5 \mathrm{~s}$ case ( solid red), which is the lowest curve.

\section{Maps from shorter survey segments}

We now consider making maps from a shorter time segment of the data. In a full year of observations, for a PLANCK-like scanning strategy, all parts of the sky are looked at two different seasons. About 7 months is needed to observe the full sky. Maps from a shorter segment cover just a part of the sky.

When maps are made from a shorter observation period, the number of crossing points is reduced and their pattern is different. Destriping 7 months of data differs qualitatively from the case of a full year, since for a large part of the sky the secondseason observations are missing. We expect the loss of the corresponding crossing points to result in a loss of output map quality due to larger baseline errors. Destriping relies then more on the crossing points which are near the ecliptic poles. When the observation period is shortened further, only part of the sky is covered. Since the crossing point structure is not necessarily changed qualitatively, we expect the map quality to worsen more slowly as a function of survey duration. Because of the cycloidal 


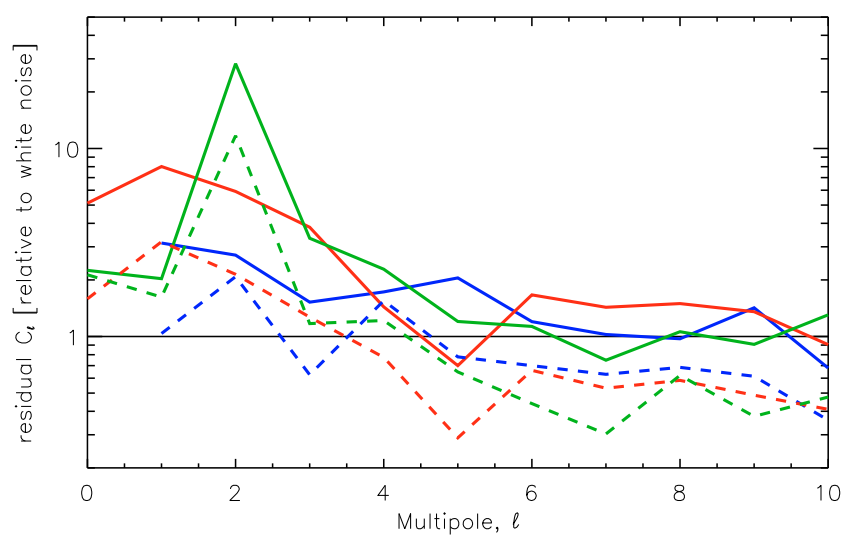

Fig. 44. Same as Fig. 39, but with the noise prior, and using a short baseline $t_{\text {base }}=2.5 \mathrm{~s}$. The Stokes parameters are now indicated by the colors: blue for $I$, red for $Q$, and green for $U$. The solid lines are for $f_{\mathrm{k}}=50 \mathrm{mHz}$, and the dashed lines for $f_{\mathrm{k}}=25 \mathrm{mHz}$.

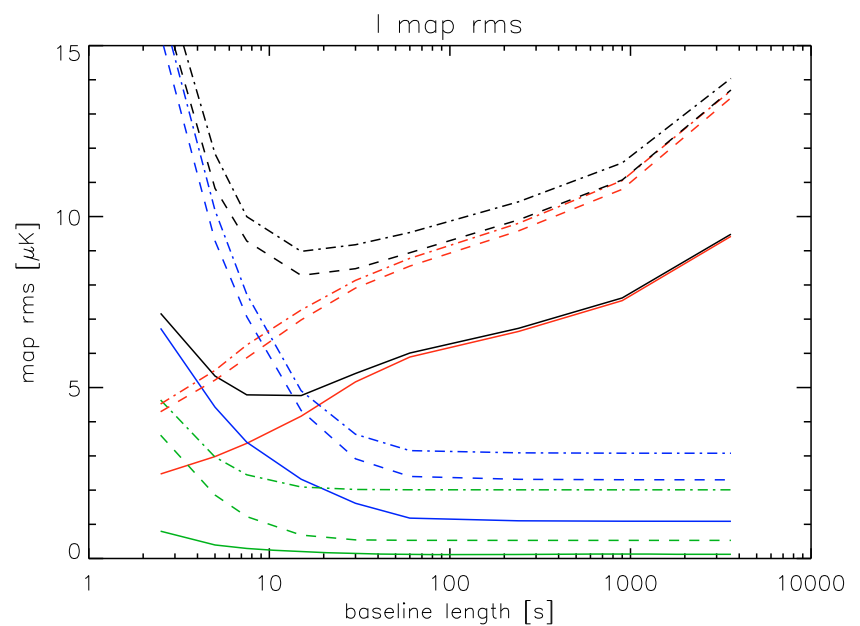

Fig. 45. Same as the bottom panel of Fig. 25, but now we have included results from 7-month (dashed) and 1-month (dot-dashed) survey segments also. The 1-month results show averages from all 12 months of the survey.

scanning strategy, the crossing point structure, however, changes with a 6-month period, so it will be different for different weeks or months.

In Fig. 45 we show the residual $I$ map rms as a function of $t_{\text {base }}$ for 1-month, 7-month, and 1-year surveys. The results for the $Q$ and $U$ maps look qualitatively the same, except that the signal baseline contribution is, of course, much lower. We have excluded all pixels with rcond $\leq 0.4$ or $n_{\text {hit }} \leq 400$ from the residual maps.

There are basically two kinds of effects contributing to these results. The more trivial effect is that of the lower hit count. The average number of hits per pixel in the 7-month survey is 7/12 of that of the full-year survey, so we expect that alone to increase the residual map rms by a factor of $\sqrt{12 / 7}=1.31$. This, indeed, accounts for most of the change in the unmodeled $1 / f$ contribution to the residual map rms. When the survey segment is shortened below 7 months, the number of included pixels falls almost in line with the number of samples, so the hit count per pixel stays now roughly constant, and there is not much additional effect when going from 7 months to 1 month.

The other effect is that of the change in the pattern of crossing points on solving the baselines. This shows clearly in the
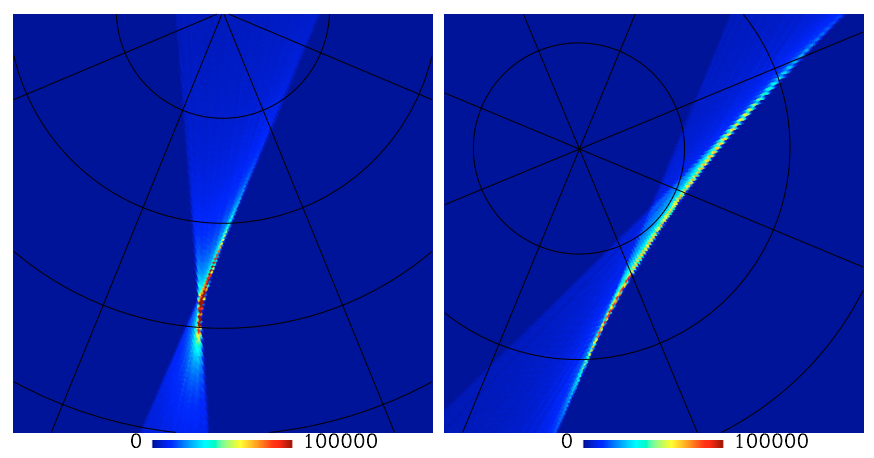

Fig. 46. Linear hit maps near the ecliptic North Pole for the one-month surveys corresponding to the first (left) and second (right) month of the full year survey.

white noise baseline and signal baseline contributions, where baseline errors were important already for the full 1-year survey. Here the change from 7 months to 1 month brings also a significant change. A closer inspection of the different months reveals, however, that it is only some of the months that are clearly worse than the 7-month case.

The first and the second month represent the two extreme cases. See Fig. 46. Note how different is the structure of crossing points between these two months. The same happens near the South Pole. For the first month we are close to the situation where all scanning circles cross each other at the same pixel. The effect of this is the most striking for the signal baseline component, for which it couples to the signal gradients in those few pixels where the circles cross and the pattern of hits within those pixels. In the second month the crossings are spread over a wide arc.

To study the effect of the length of the survey segment, and also its timewise location with respect to the cycloid, we fixed the baseline length to $t_{\text {base }}=1 \mathrm{~min}$, and considered survey segments of $1,2,4,6,8,10,16,24,32,40,48,64,128,256$, and 366 days. We also considered the effect of extending the mission to 488 days (16 months). Moreover, for each survey segment length (except the 16 month one) we considered three different starting points for the segment. To separate the effect of the change in hit count from the effect of crossing point structure on solving baselines, we plot first the rms of the binned white noise map, which has only the first effect, in Fig. 47.

The binned white noise map rms is given by $\sigma\left\langle n_{\text {hit }}^{-1}\right\rangle^{1 / 2}$, calculated over the included pixels. Thus it falls and rises in inverse relation to the mean hit count. In Fig. 47 we see it first falling, since additional rings partially overlap preceding rings and thus do not bring in new pixels to the map as fast as new samples accumulate. The rms begins to rise after the first three months since the cycloidal scanning strategy brings then the satellite spin axis south of the ecliptic, where the spin axis repointing step is larger, causing new pixels to be added to the map at a faster rate than when the spin axis was north of the ecliptic. After a little over half a year, the whole sky is covered, and new samples just increase the hit count causing the rms to fall.

To see the second effect, we consider the ratio of the residual map rms to the rms of the binned white noise map from the same data. This tells us how well we are doing compared to the white noise level. See Fig. 48. We see that for all three starting points, the relative map quality consistently improves after 128 days with further increase in the survey length up to a full year. The relative quality (with respect to binned white noise) of the 16-month map is the same as the 12-month map. 


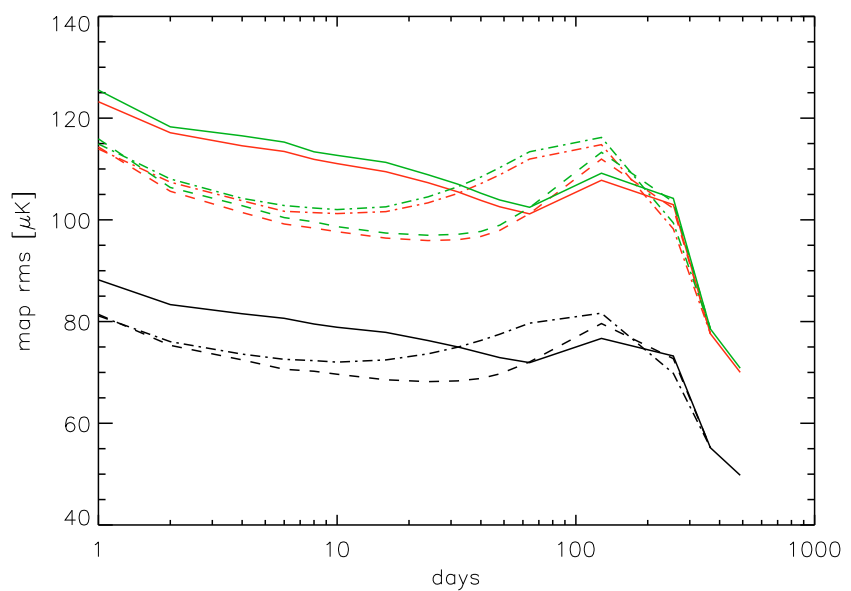

Fig. 47. Binned white noise map rms as a function of survey length for $t_{\text {base }}=1 \mathrm{~min}$. Three different starting points were considered: from the beginning of the simulated mission (solid), starting 30 days later (dashed), and starting 61 days later (dot-dashed). The black lines are for the Stokes $I$ map, red for $Q$, and green for $U$.

For shorter survey pieces the behavior as a function of survey length depends on the starting point. For $Q$ and $U$, short segments, 10 days or less, are clearly much worse than longer ones. For the $I$ map, the relative quality in some cases worsens with time up to about 40 days. This is related to the changing pattern of crossing points in the cycloidal strategy. For some periods of time the pattern is more ill-conditioned and adding data from such a period to another short period makes things worse rather than better. From Fig. 49 we can conclude that one such period is somewhere between days 1 and 24 and another somewhere between days 70 and 101 of the simulated mission, since the residual $I$ map rms is increasing during those periods. These coincide with the times when the cycloidal scanning strategy is producing a clustering of crossing points of nearby scanning rings at the corners of caustics around ecliptic poles. The baselines can be better solved from a more widely distributed set of crossing points (Wright 1996). We can see from Fig. 48, that the unmodeled $1 / f$ and reference baseline contributions stay relatively constant, so the variation indeed comes from baseline error.

\section{Conclusions}

We have described our destriping map-making method (Polar/Madam) for CMB surveys. The method has a parameter, the baseline length $t_{\text {base }}$, that affects the performance of the method. With long baselines, the Madam code is faster and requires less computer memory. The computer time and memory requirements of the code are discussed in Ashdown et al. (2009), where it is compared to other codes and methods.

Here we have done a detailed analysis of the residual errors in maps produced with the method. In this paper we concentrated on destriping without a noise prior. For short baselines the results can be improved by utilizing prior information on the noise spectrum. This will be described in (Keihänen et al. 2009).

We have divided the destriping residuals into six components. Three of them, white noise reference baselines, unmodeled $1 / f$ noise, and pixelization noise reference baselines, are easy to estimate analytically from the noise power spectrum and the signal angular power spectrum. In the map domain the baseline components appear as a superposition of thin constant
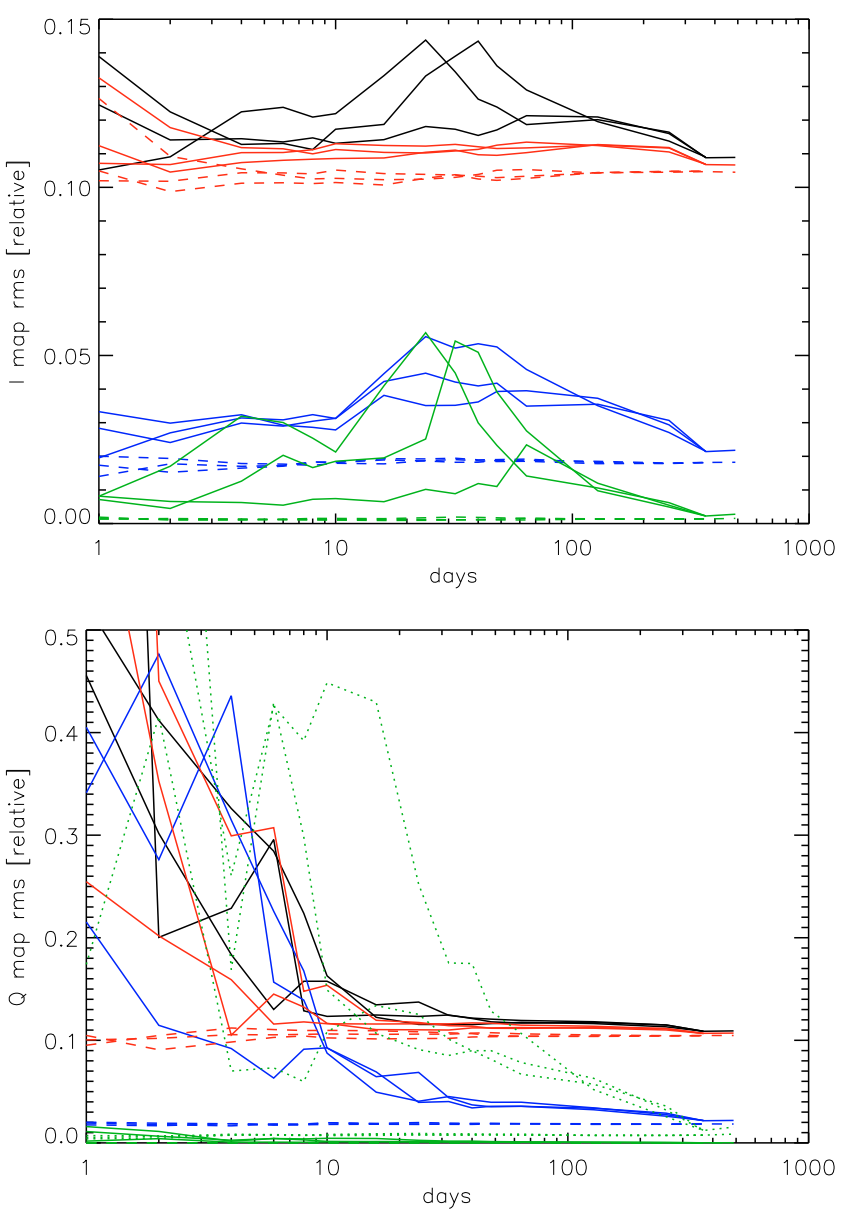

Fig. 48. Ratio of the residual map rms to the binned white noise map rms (see Fig. 47) as a function of survey length for $t_{\text {base }}=1 \mathrm{~min}$. The black line is for the full residual (excluding binned white noise), blue for the white noise baseline map, red for residual $1 / f$, and green for signal baseline map. We show also separately the reference baseline contributions (dashed). The top panel is for the Stokes parameter I, the bottom panel for $Q$. Results for $U$ are qualitatively the same as for $Q$. For $Q$, the signal baseline component is plotted multiplied by a factor of 100 also (dotted).

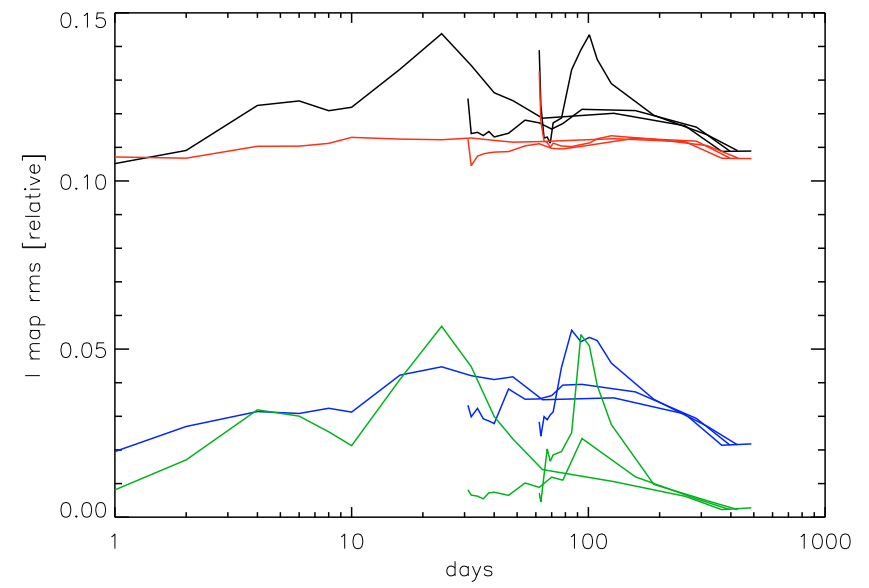

Fig. 49. The top panel of Fig. 48 replotted so that the horizontal axis corresponds to the distance of the end of the included data from the beginning of the simulated mission, so only for the case of the first starting point does it correspond to the length of the data used.

stripes of length $\theta_{\text {base }}$, whereas the unmodeled $1 / f$ noise varies along such stripes with a period comparable to $\theta_{\text {base }}$. 
The three other components are related to how accurately baselines can be solved from crossing points, and depend on the scanning strategy. These baseline error components are minimized when there are very many crossing points widely distributed. Especially when making partial sky maps from short survey segments around a time when ring crossings cluster in the same small region of the sky these errors may blow up. Since the baseline errors are correlated over long time scales, they produce wide bands in the map domain and are important for low multipoles.

The relevance of this analysis is that it guides us in what kind of noise residuals to expect in the maps for given detector noise spectra, and what baseline length to use in destriping map-making.

For long surveys, with a good distribution of crossing points, the dominant residual error components are the white noise baselines and unmodeled $1 / f$ noise. Their combined effect can be minimized, when the baseline length is chosen according to Eqs. (58) or (60), which put it close to $t_{\text {base }} \approx 1 /\left(2 f_{\mathrm{k}}\right)$, where $f_{\mathrm{k}}$ is the knee frequency of the noise. Because of the other error components, one should choose a somewhat longer baseline than this. (When a noise prior is used, shorter baselines are better.)

For a PLANCK-like scanning strategy, where the same circle of the sky is observed many times, the difference between baseline lengths from the spin period to the repointing period is mainly due to nutation, and is small when the nutation is small compared to the map pixel size. If the knee frequency is comparable to, or smaller than, the spin frequency, then the baseline length should be chosen from this range, if no noise prior is used. For a higher knee frequency the residual errors are larger, and a shorter baseline is better.

Acknowledgements. The work reported in this paper was done by the CTP Working Group of the PLANCK Consortia. PLANCK is a mission of the European Space Agency. We thank K. Górski, C. R. Lawrence, and J. P. Leahy for useful comments. This work was supported by the Academy of Finland grants 205800, $213984,214598,121703$, and 121962. We acknowledge the support by the ASI contracts "PLANCK LFI Activity of Phase E2" and I/016/07/0 "COFIS". R.K. is supported by the Jenny and Antti Wihuri Foundation. H.K.S. thanks Waldemar von Frenckells stiftelse, H.K.S. and T.P. thank the Magnus Ehrnrooth Foundation, and E.K. and T.P. thank the Väisälä Foundation for financial support. This work was supported by the European Union through the Marie Curie Research and Training Network "UniverseNet" (MRTN-CT-2006-035863). We thank CSC (Finland) for computational resources. We acknowledge use of the CAMB code for the computation of the theoretical CMB angular power spectrum. This work has made use of the PLANCK satellite simulation package (level S), which is assembled by the Max Planck Institute for Astrophysics PLANCK Analysis Centre (MPAC). Some of the results in this paper have been derived using the HEALPix package (Górski et al. 2005).

\section{References}

Armitage, C., \& Wandelt, B. D. 2005, Phys. Rev. D, 70, 123007

Ashdown, M. A. J., Baccigalupi, C., Balbi, A., et al. 2007a, A\&A, 467, 761

Ashdown, M. A. J., Baccigalupi, C., Balbi, A., et al. 2007b, A\&A, 471, 361

Ashdown, M. A. J., Baccigalupi, C., Bartlett, J. G., et al. 2009, A\&A, 493, 753
Borrill, J. 1999, in Proceedings of the 5th European SGI/Gray MPP Workshop, Bologna, Italy [arXiv: astro-ph/9911389]

Burigana, C., \& Sáez, D. 2003, A\&A, 409, 423

Burigana, C., Seiffert, M., Mandolesi, N., \& Bersanelli, M. 1997a, Int. Rep. TeSRE/CNR, 186/1997, March

Burigana, C., Malaspina, M., Mandolesi, N., et al. 1997b, Int. Rep. TeSRE/CNR, 198/1997, November [arXiv: astro-ph/9906360]

Challinor, A., Fosalba, P., Mortlock, D., et al. 2000, Phys. Rev. D, 62, 123002

Couchot, F., Delabrouille, J., Kaplan, J., \& Revenu, B. 1999, A\&AS, 135, 579

de Gasperis, G., Balbi, A., Cabella, P., Natoli, P., \& Vittorio, N. 2005, A\&A, 436, 1159

Delabrouille, J. 1998, A\&ASS, 127, 555

Doré, O., Teyssier, R., Bouchet, F. R., Vibert, D., \& Prunet, S. 2001, A\&A, 374, 358

Dupac, X., \& Tauber, J. 2005, A\&A, 430, 363

Eftstathiou, G. 2005, MNRAS, 356, 1549

Eftstathiou, G. 2007, MNRAS, 380, 1621

Ganga, K. 1994, Ph.D. Thesis,

http://spider.ipac.caltech.edu/staff/ kmg/

Goldberg, J. N., Macfarlane, A. J., Newman, E. T., Rohrlich, F., \& Sudarshan, E. C. G. 1967, J. Math. Phys. (N.Y.), 8, 2155

Górski, K. M., Wandelt, B. D., Hivon, E., Hansen, F. K., \& Banday, A. J. 2005, ApJ, 622, 759

Harrison, D. L., van Leeuwen, F., \& Ashdown, M. A. J. 2008, in preparation

Keihänen, E., Kurki-Suonio, H., Poutanen, T., Maino, D., \& Burigana, C. 2004, A\&A, 428, 287

Keihänen, E., Kurki-Suonio, H., \& Poutanen, T. 2005, MNRAS, 360, 390

Keihänen, E., Keskitalo, R., Kurki-Suonio, H., et al. 2009, A\&A, accepted [arXiv: 0907.0367]

Keskitalo, R., Ashdown, M. A. J., Cabella, P., et al. 2009, A\&A, submitted [arXiv:0906.0175]

Leahy, J. P., Bersanelli, M., D’Arcangelo, O., et al. 2009, A\&A, submitted

Maino, D., Burigana, C., Maltoni, M., et al. 1999, A\&ASS, 140, 383

Maino, D., Burigana, C., Górski, K. M., Mandolesi, N., \& Bersanelli, M. 2002, A\&A, 387, 356

Natoli, P., de Gasperis, G., Gheller, C., \& Vittorio, N. 2001, A\&A, 372, 346

Newman, E. T., \& Penrose, R. 1966, J. Math. Phys. (N.Y.), 7, 863

Planck Collaboration 2005, The Scientific Programme of PLANCK ("PlANCK Bluebook"), ESA-SCI, 2005, 1 [arXiv: astro-ph/0604069]

Poutanen, T., Maino, D., Kurki-Suonio, H., Keihänen, E., \& Hivon, E. 2004, MNRAS, 353, 43

Poutanen, T., de Gasperis, G., Hivon, E., et al. 2006, A\&A, 449, 1311

Press, W. H., Teukolsky, S. A., Wetterling, W. T., \& Flannery, B. P. 1992, Numerical Recipes, 2nd edn. (Cambridge: Cambridge University Press)

Reinecke, M., Dolag, K., Hell, R., Bartelmann, M., \& Ensslin, T. 2006, A\&A, 445,373

Revenu, B., Kim, A., Ansari, R., et al. 2000, A\&ASS, 142, 499

Sbarra, C., Carretti, E., Cortiglioni, S., et al. 2003, A\&A, 401, 1215

Seiffert, M., Mennella, A., Burigana, C., et al. 2002, A\&A, 391, 1185

Stompor, R., \& White, M. 2004, A\&A, 419, 783

Sutton, D., Johnson, B. R., Brown, M. L., et al. 2009, MNRAS, 393, 894

Tuovinen, J. 2003, PlANCK Newsletter Number 4, 7 (www.rssd.esa. int/SA/PLANCK/docs/Newsletters/PlanckNewsletter4.pdf)

van Leeuwen, F., Challinor, A. D., Mortlock, D. J., et al. 2002, MNRAS, 331, 975

Varshalovich, D. A., Moskalev, A. N., \& Khersonskii, V. K. 1988, Quantum Theory of Angular Momentum (Singapore: World Scientific)

Wandelt, B. D., \& Górski, K. M. 2001, Phys. Rev. D, 63, 123002

Wright, E. L. 1996, Report UCLA-ASTRO-ELW-96-03, November [arXiv: astro-ph/9612006]

Yvon, D., \& Mayet, F. 2005, A\&A, 436, 729

Zaldarriaga, M., \& Seljak, U. 1997, Phys. Rev. D, 55, 1830 